\title{
Geologic Framework of the Regional Ground-Water Flow System in the Upper Deschutes Basin, Oregon
}
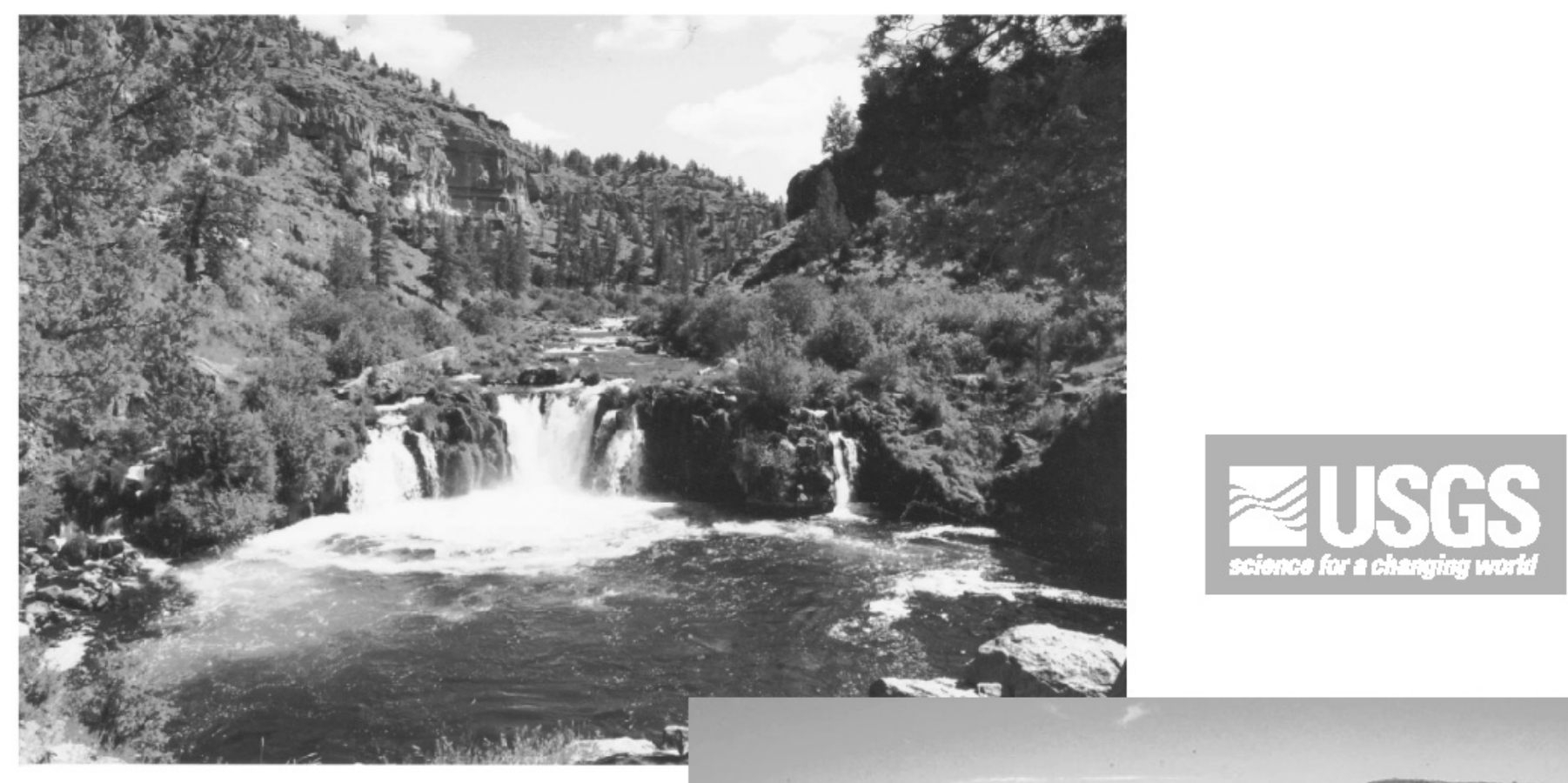

U.S. DEPARTMENT OF THE INTERIOR U.S. GEOLOGICAL SURVEY Water-Resources Investigations

Report 02-4015

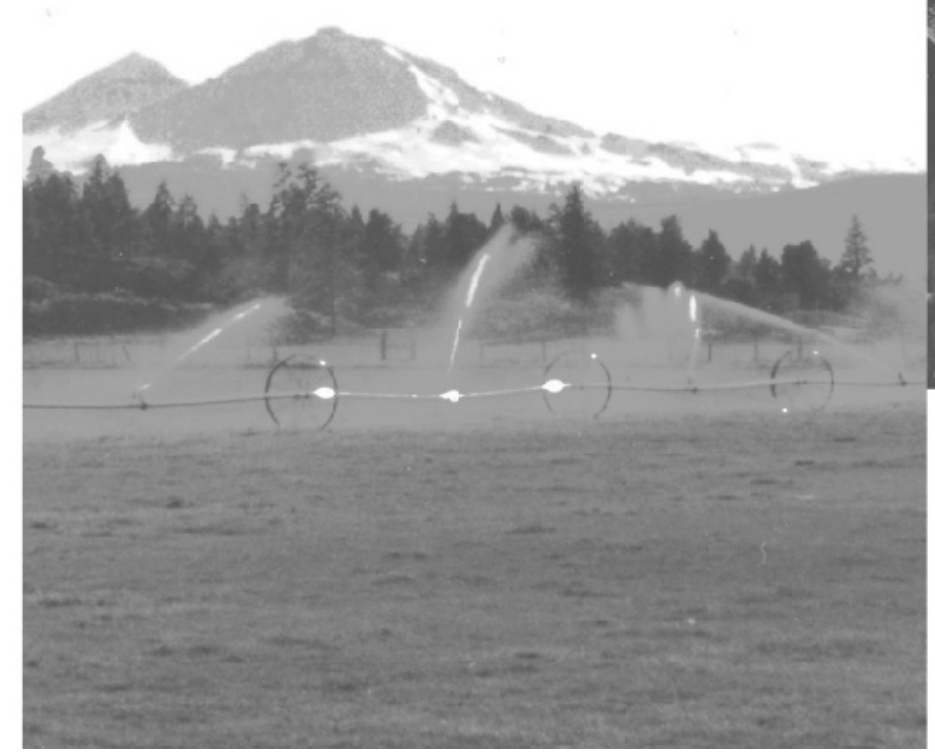

Prepared in cooperation with

OREGON WATER RESOURCES DEPARTMENT; CITIES OF BEND, REDMOND, AND SISTERS; DESCHUTES AND JEFFERSON COUNTIES; THE CONFEDERATED TRIBES OF THE

WARM SPRINGS RESERVATION OF OREGON; and U.S. ENVIRONMENTAL PROTECTION AGENCY 


\section{Cover photographs:}

Top: Steelhead Falls on the Deschutes River near Crooked River Ranch, Oregon.

Middle: Crooked River Canyon at Crooked River Ranch, Oregon.

Bottom: North and Middle Sister with a wheel-line irrigation system in the foreground near Sisters, Oregon. (Photographs by Rodney R. Caldwell, U.S. Geological Survey.) 


\section{Geologic Framework of the Regional Ground-Water Flow System in the Upper Deschutes Basin, Oregon}

By KENNETH E. LITE JR. and MARSHALL W. GANNETT

Water-Resources Investigations Report 02-4015

Prepared in cooperation with Oregon Water Resources Department;

Cities of Bend, Redmond, and Sisters; Deschutes and Jefferson Counties;

The Confederated Tribes of the Warm Springs Reservation of Oregon; and U.S. Environmental Protection Agency 


\section{U. S. DEPARTMENT OF THE INTERIOR}

GALE A. NORTON, Secretary

U.S. GEOLOGICAL SURVEY

CHARLES G. GROAT, Director

The use of trade, product, or firm names in this publication

is for descriptive purposes only and does not imply

endorsement by the U.S. Government.

For additional information:

Copies of this report may be purchased from:

District Chief

U.S. Geological Survey

10615 S.E. Cherry Blossom Dr.

USGS Information Services

Portland, OR 97216-3103

Box 25286

E-mail: info-or@usgs.gov

Denver, CO 80225-0286

Internet: http://or.water.usgs.gov

Telephone: 1-888-ASK-USGS

Suggested citation: Lite, K.E., Jr., and Gannett, M.W., 2002, Geologic framework of the regional ground-water flow system in the upper Deschutes Basin, Oregon: U.S. Geological Survey Water-Resources Investigations Report 02-4015, p. 44. 


\section{CONTENTS}

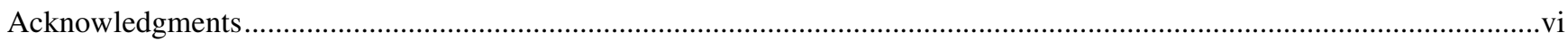

Abstract ................................

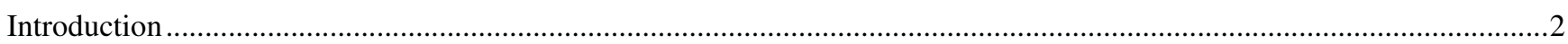

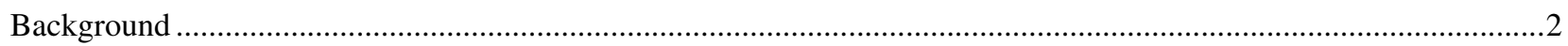

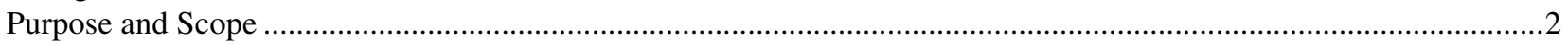

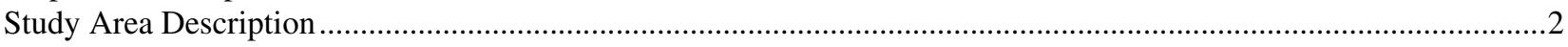

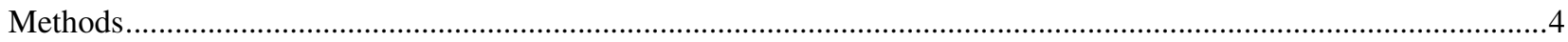

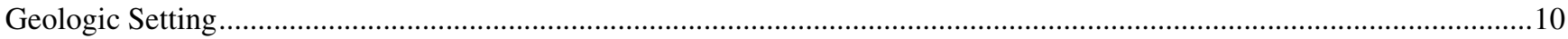

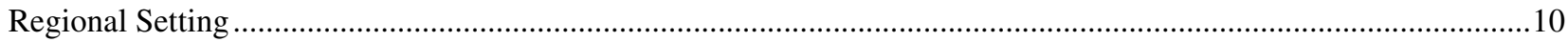

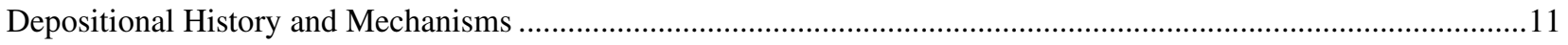

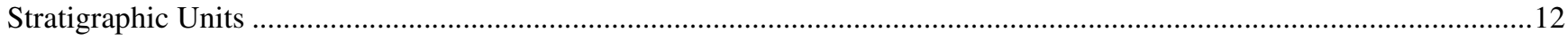

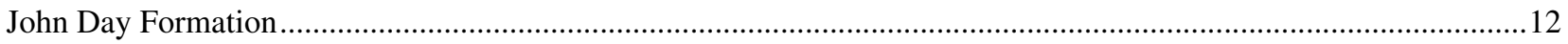

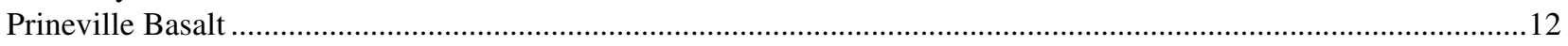

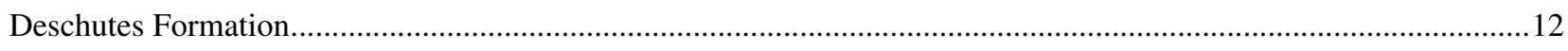

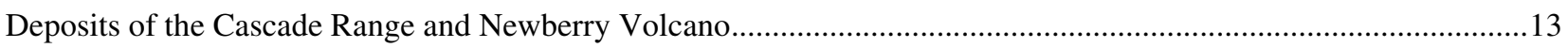

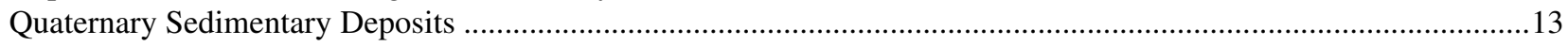

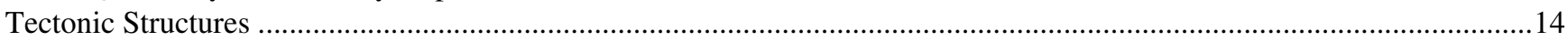

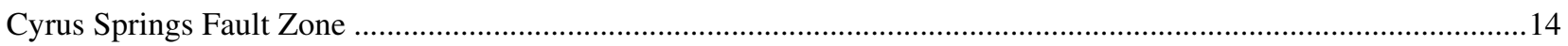

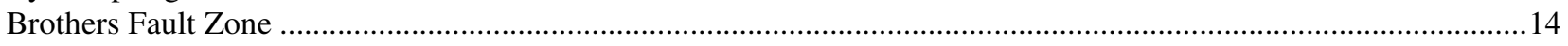

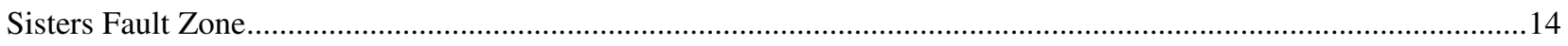

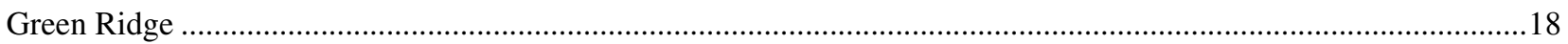

Walker Rim Fault Zone and Chemult Graben ...............................................................................................

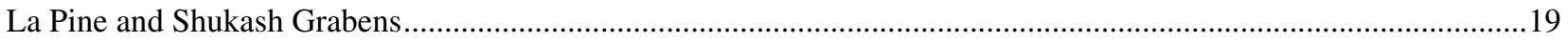

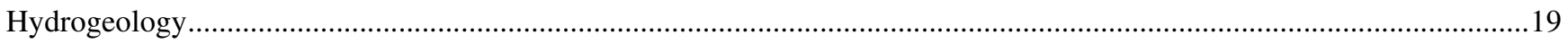

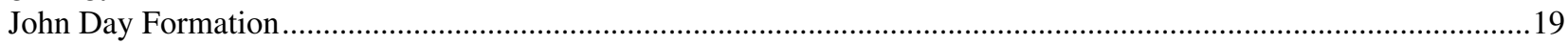

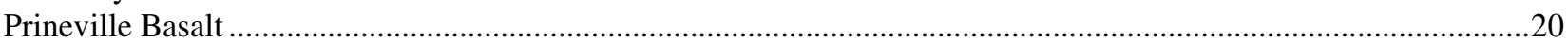

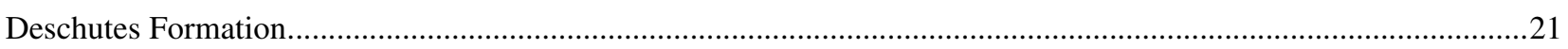

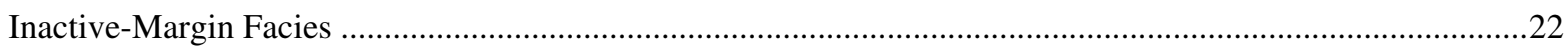

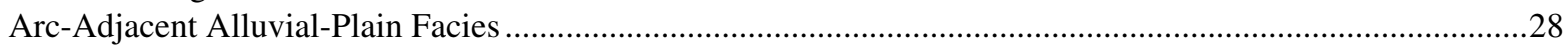

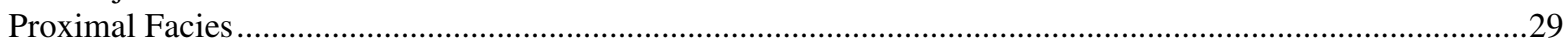

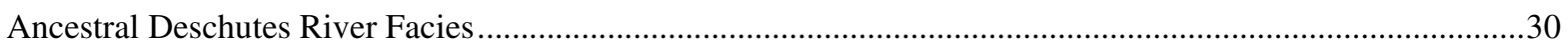

Deposits of the Cascade Range and Newberry Volcano..........................................................................................

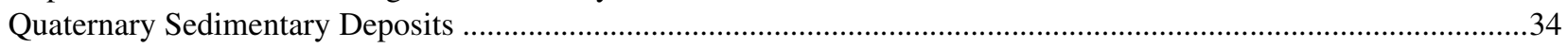

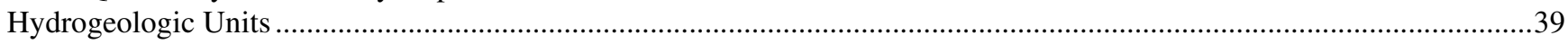

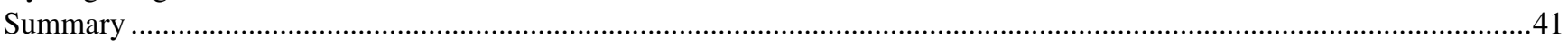

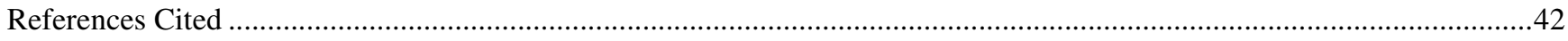

\section{PLATE [In pocket]}

1. Map and cross sections showing the generalized geology of the upper Deschutes Basin and locations of selected wells.

\section{FIGURES}

1.- 5. Maps showing:

1. Location of the upper Deschutes Basin, Oregon, and major geographic and cultural features ............................

2. Location of field-located wells and land ownership in the upper Deschutes Basin, Oregon ...............................5

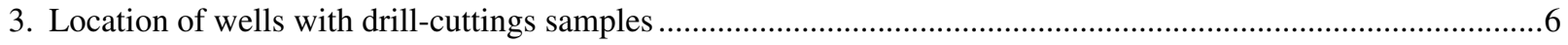

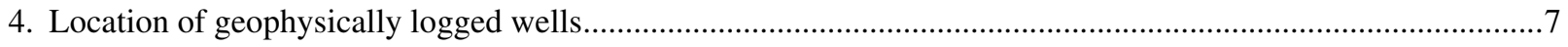

5. Location of the Deschutes Basin in relation to the physiographic provinces of Oregon .....................................10

6. Diagrammatic section through a volcanic arc and adjacent area showing the relation between volcanic and sedimentary facies. 
7.- 11. Maps showing:

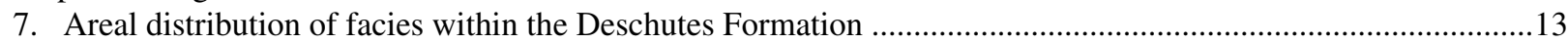

8. Major tectonic structures in the upper Deschutes Basin, Oregon ................................................................15

9. Geologic units, fault traces, and hydraulic head in wells in the vicinity of the Brothers fault zone,

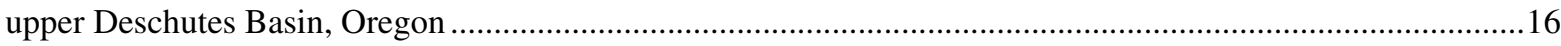

10. Generalized lines of equal hydraulic head, ground-water flow directions, and major tectonic structures in the upper Deschutes Basin, Oregon ..........................................................................................17

11. Thickness of Quaternary sediment in field-located wells and the approximate extent of saturated sediment in the Sisters area, upper Deschutes Basin, Oregon ............................................................................18

12. Geophysical logs showing natural gamma for wells constructed into the John Day Formation ...............................21

13. Geophysical log showing natural gamma for a well constructed into the Prineville Basalt near

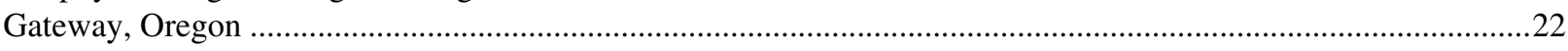

14. Map showing extent of surface exposures of the Deschutes Formation and age-equivalent deposits .......................27

15. Geophysical log showing natural gamma for a well constructed into the inactive-margin facies of

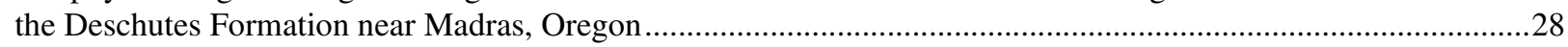

16. Geophysical logs showing natural gamma for wells constructed into the arc-adjacent alluvial-plain facies of the Deschutes Formation.....

17. Geophysical log showing natural gamma for a well constructed into layered lava flows within the Deschutes Formation southwest of Powell Buttes, upper Deschutes Basin, Oregon ..........................................30

18. Map showing extent of deposits of the Cascade Range and Newberry Volcano ..................................................32

19. Geophysical logs showing natural gamma for wells penetrating Quaternary lava flows from

Newberry Volcano and underlying Deschutes Formation strata ...........................................................................33

20. Map showing distribution and thickness of Quaternary sediment .....................................................................35

21.-22. Geophysical logs showing:

21. Natural gamma logs for wells penetrating Quaternary outwash deposits and lava flows .................................36

22. Natural gamma logs for wells penetrating Quaternary basin-fill deposits in the La Pine subbasin .....................38

23. Hydrogeologic units in the upper Deschutes Basin, Oregon ..............................................................................

\section{TABLES}

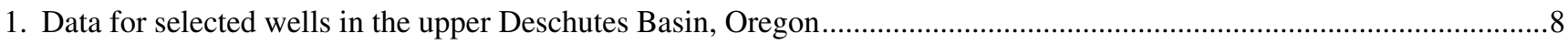

2. Major oxide analyses of cuttings samples from selected wells in the upper Deschutes Basin, Oregon ...........................23

3. Trace element analyses of cuttings samples from selected wells in the upper Deschutes Basin, Oregon ........................25

\section{CONVERSION FACTORS AND VERTICAL DATUM}

\begin{tabular}{rll}
\hline Multiply & By & To obtain \\
\hline inch (in.) & 25.4 & millimeter $(\mathrm{mm})$ \\
foot $(\mathrm{ft})$ & 0.3048 & meter $(\mathrm{m})$ \\
foot per day $(\mathrm{ft} / \mathrm{d})$ & $3.528 \times 10^{-6}$ & meters per second $(\mathrm{m} / \mathrm{s})$ \\
mile $(\mathrm{mi})$ & 1.609 & kilometer $(\mathrm{km})$ \\
square mile $\left(\mathrm{mi}^{2}\right)$ & 2.590 & square kilometer $\left(\mathrm{km}^{2}\right)$ \\
cubic foot per second $\left(\mathrm{ft}^{3} / \mathrm{s}\right)$ & 0.02832 & cubic meter per second $\left(\mathrm{m}^{3} / \mathrm{s}\right)$ \\
gallon per minute $(\mathrm{gal} / \mathrm{min})$ & $6.308 \times 10^{-5}$ & cubic meters per second $\left(\mathrm{m}^{3} / \mathrm{s}\right)$ \\
& & \\
\hline
\end{tabular}

Temperature in degrees Fahrenheit $\left({ }^{\circ} \mathrm{F}\right)$ may be converted to degrees Celsius $\left({ }^{\circ} \mathrm{C}\right)$ as follows:

$$
{ }^{\circ} \mathrm{C}=\left({ }^{\circ} \mathrm{F}-32\right) / 1.8
$$

Temperature in degrees Celsius $\left({ }^{\circ} \mathrm{C}\right)$ may be converted to degrees Fahrenheit $\left({ }^{\circ} \mathrm{F}\right)$ as follows:

$$
{ }^{\circ} \mathrm{F}=\left(1.8 \times{ }^{\circ} \mathrm{C}\right)+32
$$

Elevation: In this report, elevation is referenced to the National Geodetic Vertical Datum of 1929 (NGVD of 1929)—a geodetic datum derived from a general adjustment of the first-order level nets of both the United States and Canada, formerly called Sea Level Datum of 1929. 


\section{LOCATION SYSTEM}

The system used for locating wells, springs, and surface-water sites in this report is based on the rectangular system for subdivision of public land. The State of Oregon is divided into townships of 36 square miles numbered according to their location relative to the east-west Willamette baseline and a north-south Willamette meridian. The position of a township is given by its north-south "Township" position relative to the baseline and its east-west "Range" position relative to the meridian. Each township is divided into 36 one-square-mile (640-acre) sections numbered from 1 to 36 . For example, a well designated as 18S/11E-29AAC is located in Township 18 south, Range 11 east, section 29. The letters following the section number correspond to the location within the section; the first letter (A) identifies the quarter section (160 acres); the second letter (A) identifies the quarter-quarter section (40 acres); and the third letter (C) identifies the quarter-quarter-quarter section (10 acres). Therefore, well 29AAC is located in the SW quarter of the NE quarter of the NE quarter of section 29. When more than one designated well occurs in the quarter-quarter-quarter section, a serial number is included.

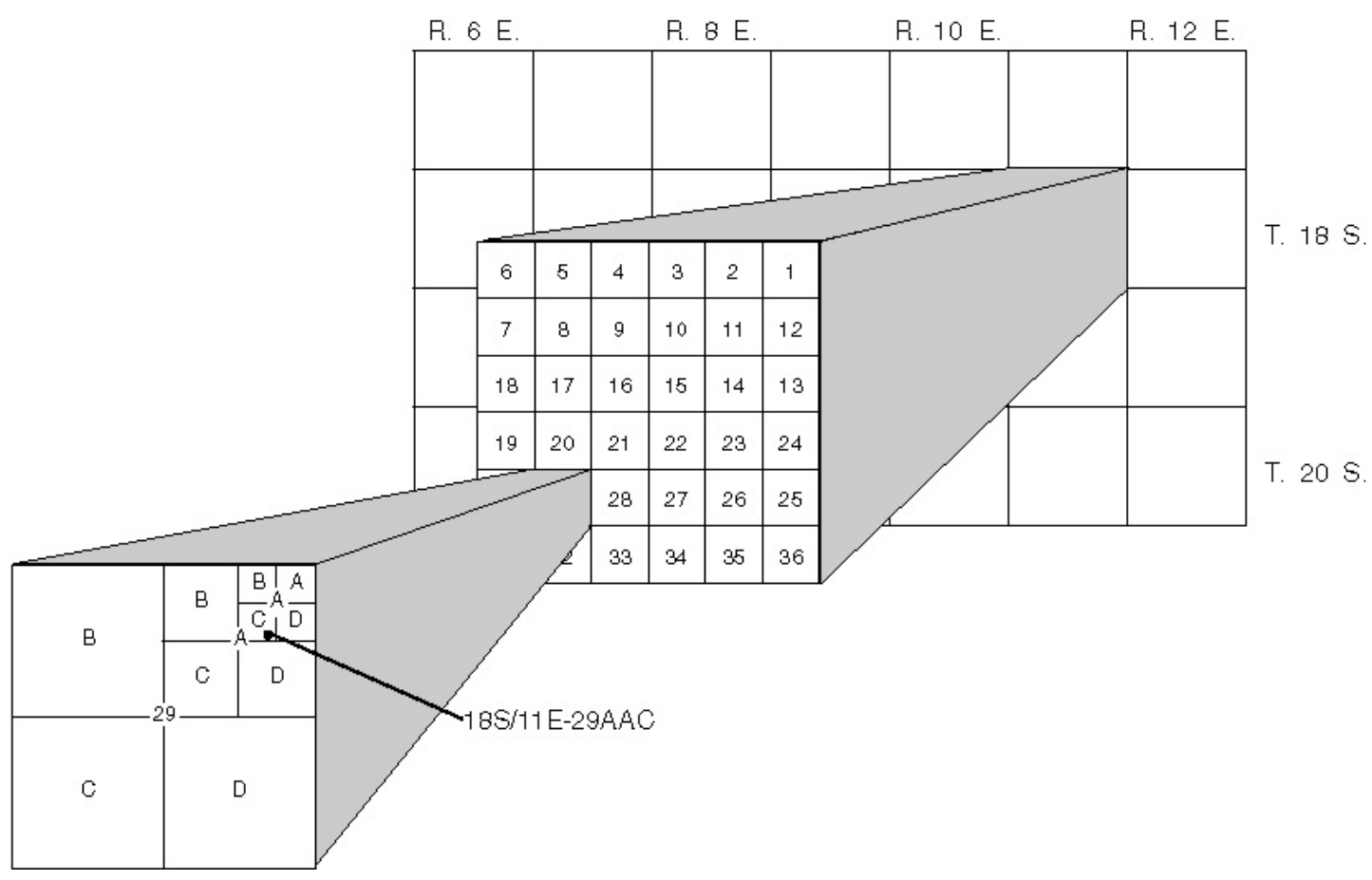

Well- and spring-location system.

Each well is assigned a unique 8-digit identification number known as the log-id number. The first two digits of the log-id number indicate the county code from the Federal Information Processing Standards (FIPS) code file for the county in which the well exists. The FIPS codes for the counties in the study area are as follows: 13, Crook County; 17, Deschutes County; 31, Jefferson County; and 35, Klamath County. The last 6 digits of the number correspond to the State of Oregon well-log number (a unique number assigned by the Oregon Water Resources Department to the report filed by the well driller).

MAPPING SOURCES:

Base map modified from U.S. Geological Survey 1:500,000 State base map, 1982, with digital data from U.S. Bureau of the Census, TIGER/Line (R), 1990, and U.S. Geological Survey Digital Line Graphs published at 1:100,000.

Projection: Universal Transverse Mercator projection, Zone 10, 1927 North American Datum. 


\section{Acknowledgments}

The authors wish to acknowledge the area residents, municipalities, private water companies, engineering companies, and well constructors who provided drill cutting samples, access to their property, and access to wells for geophysical logging. In particular we thank Doug Aiken, well constructor, who provided excellent quality samples from numerous wells throughout Deschutes County and Larry Carey, Oregon Water Resources Department, South Central Region Office, for organizing the drill cuttings sampling program and retrieving and labeling hundreds of samples. We also thank Pat Dorning, City of Redmond, and Bob MacRostie, Deschutes Valley Water Company, for providing drill cutting samples, geophysical logs, and hydrologic data from their newly constructed wells. Finally, we wish to thank the geologists that have collected much of the geologic information for the area and freely shared their insights with us. They include Dave Sherrod, U.S. Geological Survey; Rick Conrey, Washington State University; Mark Ferns, Oregon Department of Geology and Mineral Industries; Gary Smith, University of New Mexico; Ed Taylor, Oregon State University; and Larry Chitwood, Deschutes National Forest. 


\title{
Geologic Framework of the Regional Ground-Water Flow System in the Upper Deschutes Basin, Oregon
}

\author{
By Kenneth E. Lite Jr. and Marshall W. Gannett
}

\begin{abstract}
Ground water is increasingly relied upon to satisfy the needs of a growing population in the upper Deschutes Basin, Oregon. Hydrogeologic studies are being undertaken to aid in management of the ground-water resource. An understanding of the geologic factors influencing ground-water flow is basic to those investigations. The geology of the area has a direct effect on the occurrence and movement of ground water. The permeability and storage properties of rock material are influenced by the proportion, size, and degree of interconnection of open spaces the rocks contain. These properties are the result of primary geologic processes such as volcanism and sedimentation, as well as subsequent processes such as faulting, weathering, or hydrothermal alteration. The geologic landscape in the study area evolved during about 30 million years of volcanic activity related to a north-south trending volcanic arc, the current manifestation of which are today's Cascade Range volcanoes.
\end{abstract}

The oldest rock unit in the upper Deschutes Basin study area, the John Day Formation, is a sequence of upper Eocene to lower Miocene volcanic and sedimentary rocks. Weathering and alteration of the rocks has resulted in very low permeability; consequently, the unit forms the hydrologic basement for the regional groundwater flow system throughout much of the area. The Deschutes Formation and age-equivalent deposits that overlie the John Day Formation, in contrast, are highly permeable and are the most widely used ground-water-bearing units in the study area. The Deschutes Formation consists of a variety of volcanic and sedimentary deposits ranging in age from late Miocene to Pliocene (approximately 7.5 to 4.0 million years). Three distinct depositional environments previously described for the formation provide useful hydrogeologic subdivisions. The ancestral Deschutes
River deposits and some units within the arcadjacent alluvial-plain region are among the highest yielding units within the Deschutes Formation, with some wells producing up to a few thousand gallons per minute. Opal Springs basalt, Pelton basalt, and the rhyodacite dome complex near Steelhead Falls are particularly productive subunits within the Deschutes Formation and provide tens to hundreds of cubic feet per second of ground-water discharge to the Deschutes and Crooked Rivers, upstream of Round Butte Dam.

Most ground-water recharge in the upper Deschutes Basin occurs in Quaternary deposits of the Cascade Range and Newberry Volcano. These deposits are highly permeable, and the fractured character of the lava flows facilitates rapid infiltration of precipitation and snowmelt, as well as movement of ground water to lower elevations. Additional recharge from canal leakage occurs along sections of unlined canals near Bend, constructed on lava flows from Newberry Volcano. Hydrothermal alteration and secondary mineralization at depth beneath the Cascade Range and Newberry Volcano has drastically reduced the permeability of the material in those regions, effectively restricting most ground water to the strata above the altered rocks. The top of the hydrothermally altered region is considered the base of the regional ground-water system beneath the Cascade Range and Newberry Volcano.

Structural features influence ground-water flow within the upper Deschutes Basin mainly by juxtaposing materials with contrasting permeability. This juxtaposition can be caused by fault movement or by the influence of a fault on subsequent deposition. Several depositional centers have formed along the base of fault-line scarps or in grabens within the study area, and the infilling sedimentary deposits have permeability that differs from the surrounding rocks. The effects of faults on ground-water flow may be masked in some areas. 
For example, the water-table gradient changes slope in the vicinity of the Sisters fault zone, but the slope change also corresponds with a major precipitation gradient change; therefore, any influence of the fault zone is unclear.

Geologic units in the Deschutes Basin were divided into several distinct hydrogeologic units. In some instances the units correspond to existing stratigraphic divisions. In other instances, hydrogeologic units correspond to different facies within a single stratigraphic unit or formation. The hydrogeologic units include Quaternary sediment, deposits of the Cascade Range and Newberry Volcano, four zones within the Deschutes Formation and age-equivalent rocks that roughly correspond with depositional environments, and pre-Deschutes-age strata.

\section{INTRODUCTION}

\section{Background}

The population in the upper Deschutes Basin has grown rapidly during the past few decades. Deschutes County, the most populous county in the basin, experienced a population increase of from 30,442 in 1970 to 106,700 in 1999 (State of Oregon, 2001). This growth is expected to continue. Surface-water resources in the area have been virtually closed to additional appropriation for many years by the State of Oregon. Ground water, therefore, is increasingly relied upon to satisfy the needs of the growing population.

To provide information on the ground-water resources of the upper Deschutes Basin, the U.S. Geological Survey (USGS) began a cooperative study in 1993 with the Oregon Water Resources Department (OWRD); the Cities of Bend, Redmond, and Sisters; Deschutes and Jefferson Counties; The Confederated Tribes of the Warm Springs Reservation of Oregon; and the U.S. Environmental Protection Agency. The objectives of the study were to provide a quantitative assessment of the regional ground-water system and to provide the understanding and analytical tools for enabling State and local government agencies, as well as the general public, to make informed resourcemanagement decisions. This report is one in a series that will present the results of the upper Deschutes Basin ground-water study.

\section{Purpose and Scope}

The purpose of this report is to describe the various geologic structures and stratigraphic units that form the framework for the ground-water flow system in the upper Deschutes Basin. The geology has a direct effect on the occurrence and movement of ground water. The proportion, size, and degree of interconnection of void spaces within a geologic material influence the water-bearing properties of that material. The proportion of void space and the degree of interconnection of geologic material are often the result of primary geologic processes, such as volcanic eruptions and sedimentation, as well as subsequent processes, such as faulting and weathering. Understanding the geologic framework of the area and ascertaining the relation between permeability and geologic processes is fundamental to understanding the ground-water flow system.

This report will present a context for relating sitespecific geologic and hydrologic data to the regional geologic framework and, thus, the regional groundwater flow system. The report progresses from a description of the volcano-tectonic evolution of the study area, through a description of important waterbearing rock units, and concludes with a discussion of the division of the regional geology into hydrogeologic units.

\section{Study Area Description}

The upper Deschutes Basin study area encompasses approximately $4,500 \mathrm{mi}^{2}$ (square miles) of the Deschutes River drainage basin in central Oregon (fig. 1). The area is drained by the Deschutes River and its major tributaries: the Little Deschutes River, Tumalo and Squaw Creeks, and the Metolius River from the west, and the Crooked River from the east. The mean annual flow of the Deschutes River below the tributaries (near Madras) is $4,553 \mathrm{ft}^{3} / \mathrm{s}$ (cubic foot per second). Land-surface elevation ranges from less than 1,300 ft (feet) above sea level near Gateway in the northern part of the study area to $10,358 \mathrm{ft}$ in the Cascade Range.

The study area boundaries were chosen to coincide as much as possible with natural hydrologic boundaries across which ground-water flow can be reasonably estimated or assumed to be negligible. The study area is bounded on the north by Jefferson Creek, the Metolius River, the Deschutes River, and Trout Creek; on the east by the generalized contact 


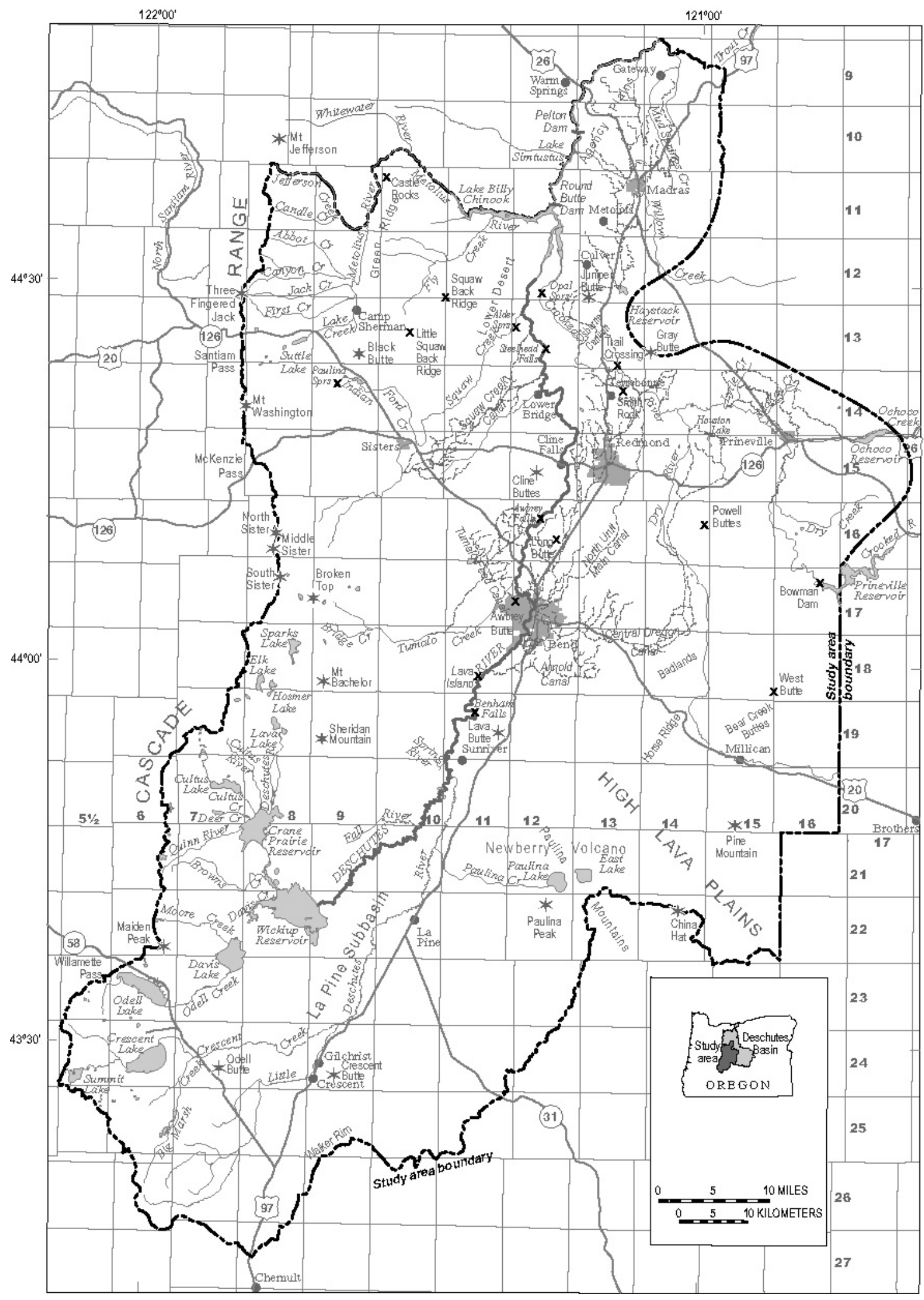

Figure 1. Location of the upper Deschutes Basin, Oregon, and major geographic and cultural features. 
between the Deschutes Formation and the older, relatively impermeable John Day Formation; on the south by the drainage divide between the Deschutes Basin and the Fort Rock and Klamath Basins; and on the west by the Cascade Range crest.

The study area includes the major population centers in the basin, where ground-water development is most intense and resource-management questions are most urgent. The major communities include Bend, Redmond, Sisters, Madras, Prineville, and La Pine. Principal industries in the region are agriculture, forest products, tourism, and service industries.

Sixty-six percent of the $4,500 \mathrm{mi}^{2}$ upper Deschutes Basin is publicly owned (fig. 2). Approximately $2,230 \mathrm{mi}^{2}$ are under the jurisdiction of the U.S. Forest Service, $730 \mathrm{mi}^{2}$ are under the jurisdiction of the Bureau of Land Management, and about $20 \mathrm{mi}^{2}$ are under the stewardship of State or County agencies. The remaining $1,520 \mathrm{mi}^{2}$ are in private ownership.

The highest elevations in the upper Deschutes Basin are in the western and southern parts. These regions are covered by coniferous forests, most of which have been managed for timber production. The remaining parts of the basin, which are at lower elevation, are more arid and, where not cultivated, are dominated by grassland, sagebrush, and juniper. Most of the non-forest-related agriculture occurs in the central and northern parts of the upper Deschutes Basin.

There are approximately 164,000 acres $\left(256 \mathrm{mi}^{2}\right)$ of irrigated agricultural land in the study area. The largest source of irrigation water is the Deschutes River. Most water is diverted from the Deschutes River near Bend and distributed to areas to the north through several hundred miles of canals. Smaller amounts of irrigation water are diverted from Tumalo and Squaw Creeks, the Crooked River, and Ochoco Creek.

The climate in the upper Deschutes Basin is controlled primarily by air masses that move eastward from the Pacific Ocean, across western Oregon, and into central Oregon. The climate is moderate, with cool, wet winters and warm, dry summers. Orographic processes result in large amounts of precipitation in the Cascade Range in the western part of the basin, with precipitation locally exceeding $200 \mathrm{in} / \mathrm{yr}$, mostly as snow during the winter (Taylor, 1993). Precipitation rates diminish rapidly toward the east to less than $10 \mathrm{in} / \mathrm{yr}$ (inches per year) in the central part of the basin. Temperatures also vary across the basin. Records from the Oregon Climate Service show that mean daily minimum and maximum temperatures at
Santiam Pass in the Cascade Range (period of record 1961-85) range from 21 and $34^{\circ} \mathrm{F}$ (degrees Fahrenheit) in January to 43 and $73^{\circ} \mathrm{F}$ in July (Oregon Climate Service, 1999). Conditions are warmer at lower elevations in the central part of the basin. The mean daily minimum and maximum temperatures in Bend (period of record 1961 to 1999 ) range from 22 and $42^{\circ} \mathrm{F}$ in January to 45 and $81^{\circ} \mathrm{F}$ in July (Oregon Climate Service, 1999).

\section{Methods}

The observations and conclusions described in this report are based on preexisting data and analyses as well as new information. The preexisting information includes well reports for approximately 15,000 wells, geophysical logs for 15 wells, and numerous geologic studies by State, Federal, university, and private consulting geologists. The scope of these studies range from site-specific municipal well designs to regional geophysical studies and geologic mapping. Much of the original geologic mapping for the area has been incorporated in a few regional-scale geologic map compilations, which are the main source of the geologic mapping data shown on the geologic map in this report (plate 1).

Newly acquired data, much of it described herein, are from wells. Those data include material descriptions for 1,500 wells that were visited and documented during this study (fig. 2) (also, see Caldwell and Truini, 1997); tens of cross-sections constructed and analyzed, based on the visited-wells data; examination of well cuttings samples from 35 wells (fig. 3); down-hole geophysical logging of 35 wells (fig. 4); and geochemical analysis of 50 well cuttings samples. In addition, numerous ground traverses were made to examine exposures of geologic materials in many parts of the study area.

Wells where cuttings samples were collected, geophysical logs were made, or from which data were cited in this report are listed in table 1. Table 1 also includes well location, wellhead elevation, and well depth information. Each well is assigned a unique eight-digit identification number known as the "log-id" number. The first two digits of the log-id number indicate the county code from the Federal Information Processing Standards (FIPS) code file for the county in which the well exists. The FIPS codes for the study area are as follows: 13, Crook County; 17, Deschutes County; 31, Jefferson County; and 35, Klamath County. The last six digits of the number correspond to the State of Oregon well-log number (a unique number assigned by the OWRD to the report filed by the well driller). 


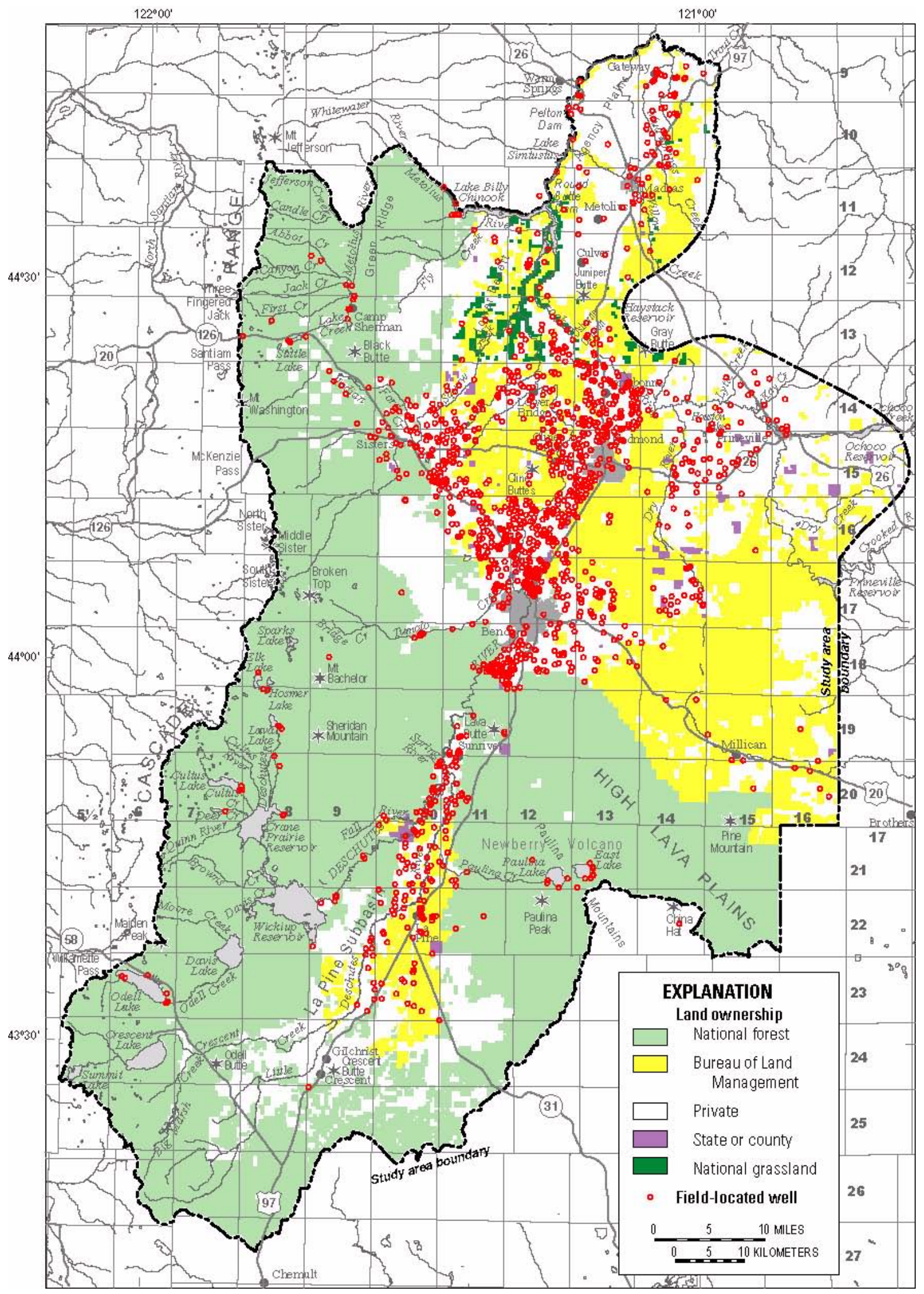

Figure 2. Location of field-located wells and land ownership in the upper Deschutes Basin, Oregon. 


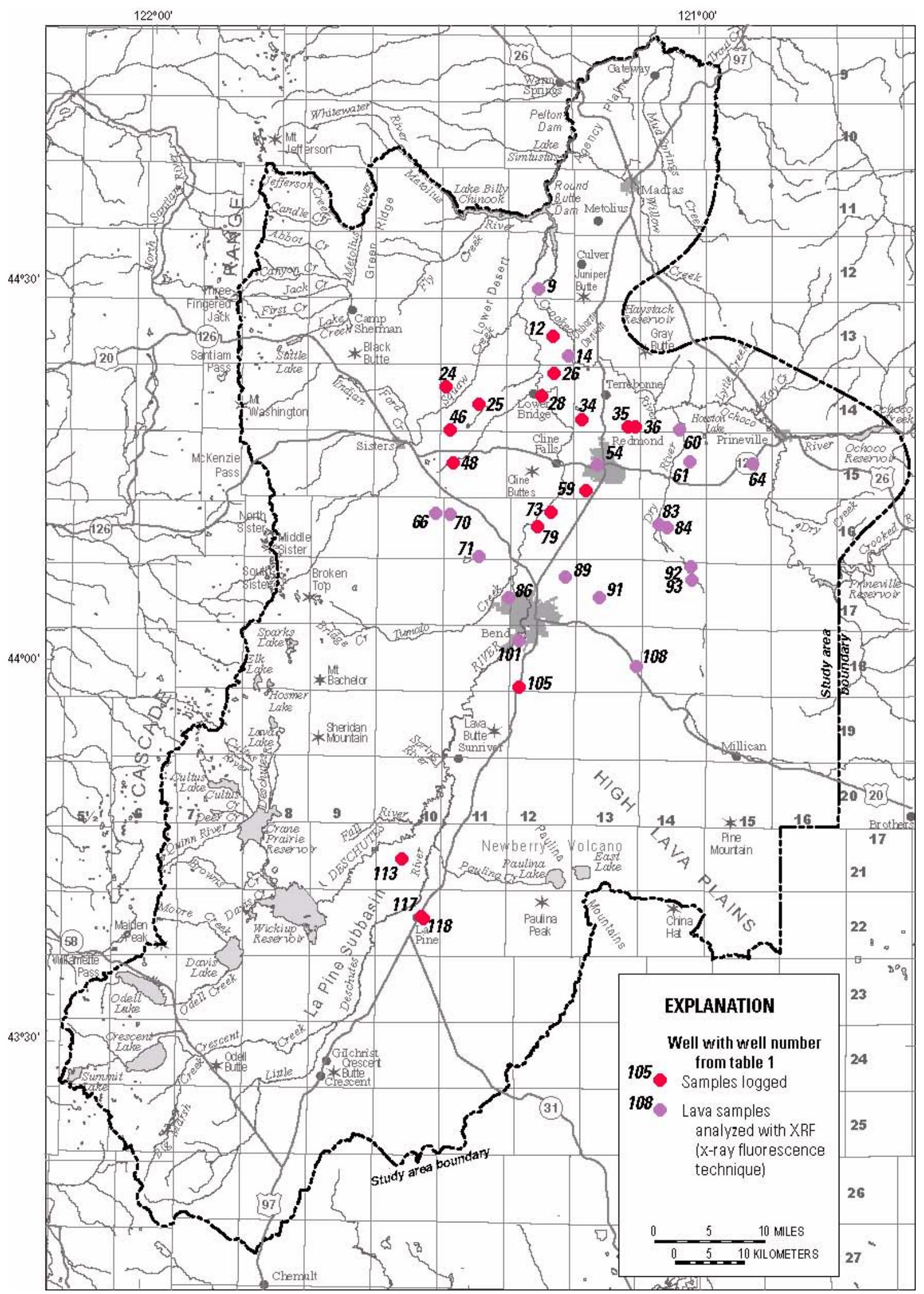

Figure 3. Location of wells with drill-cuttings samples. 


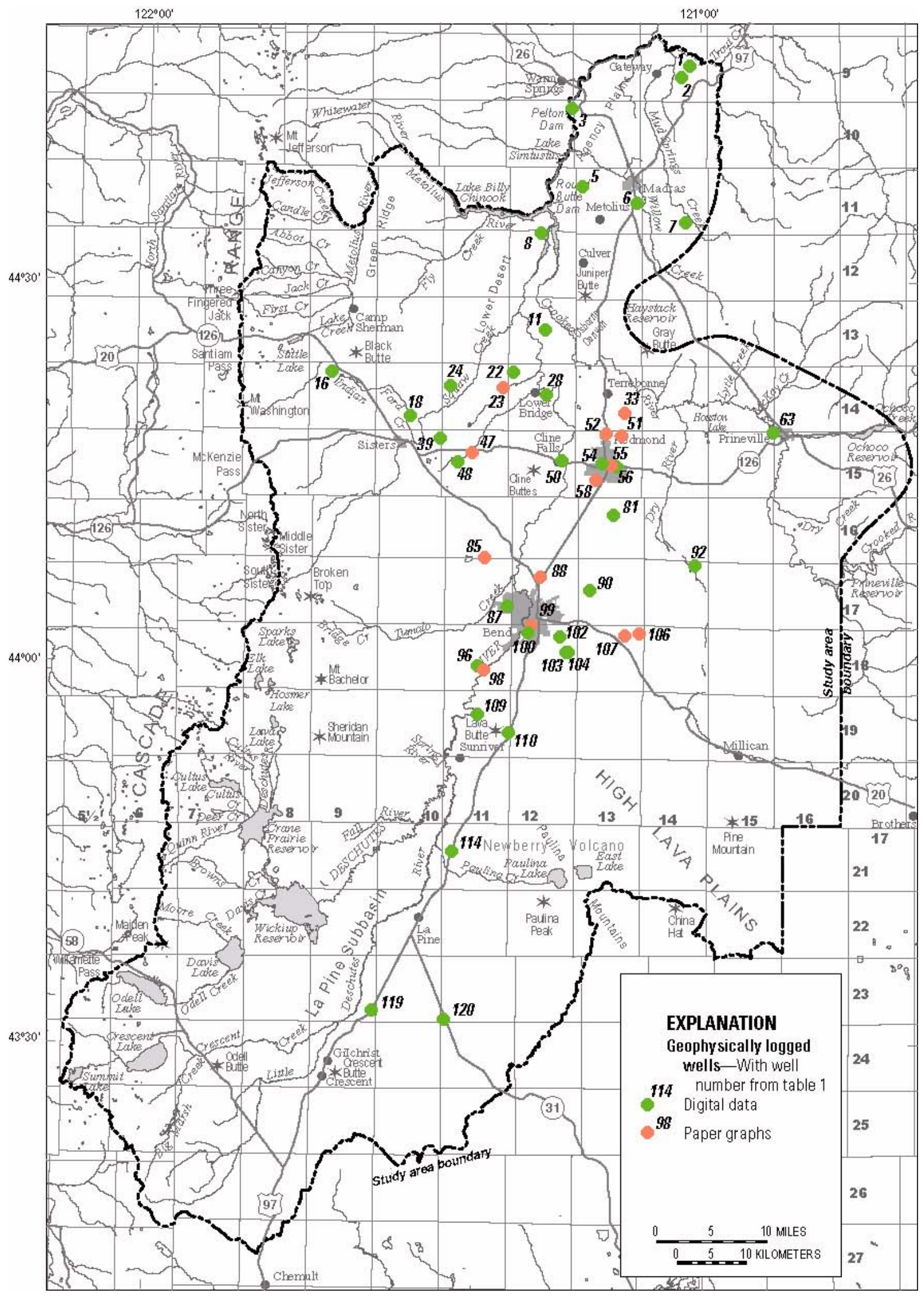

Figure 4. Location of geophysically logged wells. 
Table 1. Data for selected wells in the upper Deschutes Basin, Oregon

Well location: Refer to "Well- and "Spring-Location System" for explanation. Log-ID number: Identification number permanently assigned to the well by the U.S. Geological Survey. Latitude/Longitude of well in ${ }^{\circ}$, degrees; ', minutes; ”, seconds. Well elevation: Given in feet above sea level. Well depth: Depth of completed well, in feet below land surface. - , no sample or no data collected; X, sample collected.

\begin{tabular}{|c|c|c|c|c|c|c|c|c|c|c|}
\hline $\begin{array}{c}\text { Well } \\
\text { number }\end{array}$ & Well location & $\begin{array}{l}\text { Log-ID } \\
\text { number }\end{array}$ & Latitude & Longitude & $\begin{array}{c}\text { Well } \\
\text { elevation } \\
\text { (feet) }\end{array}$ & $\begin{array}{l}\text { Well } \\
\text { depth } \\
\text { (feet) }\end{array}$ & $\begin{array}{l}\text { Cuttings } \\
\text { samples }\end{array}$ & $\begin{array}{c}\text { Rock } \\
\text { chemistry }\end{array}$ & $\begin{array}{c}\text { Geo- } \\
\text { physical } \\
\text { log }\end{array}$ & $\begin{array}{c}\text { Aquifer } \\
\text { test }\end{array}$ \\
\hline 1 & 09S/14E-14CCC & 31000219 & $44^{\circ} 46^{\prime} 49.28^{\prime \prime}$ & $121^{\circ} 01^{\prime} 46.48^{\prime \prime}$ & 1,962 & 550 & - & - & $\mathrm{X}$ & - \\
\hline 2 & 09S/14E-27BBA & 31000102 & $44^{\circ} 45^{\prime} 56.78^{\prime \prime}$ & $121^{\circ} 02^{\prime} 46.50^{\prime \prime}$ & 1,885 & 285 & - & - & $\mathrm{X}$ & - \\
\hline 3 & $10 \mathrm{~S} / 12 \mathrm{E}-01 \mathrm{CDD} 2$ & 31000125 & $44^{\circ} 43^{\prime} 29.42^{\prime \prime}$ & $121^{\circ} 14^{\prime} 43.44^{\prime \prime}$ & 1,455 & 910 & - & - & $\mathrm{X}$ & - \\
\hline 4 & 11S/13E-01BCA2 & 31000427 & $44^{\circ} 38^{\prime} 48.47^{\prime \prime}$ & $121^{\circ} 07^{\prime} 27.30^{\prime \prime}$ & 2,278 & 451 & - & - & - & $\mathrm{X}$ \\
\hline 5 & 11S/13E-07CCD1 & 31000435 & $44^{\circ} 37^{\prime} 21.23^{\prime \prime}$ & $121^{\circ} 13^{\prime} 34.25^{\prime \prime}$ & 2,680 & 835 & - & - & $\mathrm{X}$ & - \\
\hline 6 & 11S/13E-24BCD1 & 31000466 & $44^{\circ} 36^{\prime} 02.05^{\prime \prime}$ & $121^{\circ} 07^{\prime} 33.30^{\prime \prime}$ & 2,490 & 590 & - & - & $\mathrm{X}$ & - \\
\hline 7 & 11S/14E-34ABC & 31000918 & $44^{\circ} 34^{\prime} 32.70^{\prime \prime}$ & $121^{\circ} 02^{\prime} 16.00^{\prime \prime}$ & 2,990 & 303 & - & - & $\mathrm{X}$ & - \\
\hline 8 & 12S/12E-04BDD1 & 31000518 & $44^{\circ} 33^{\prime} 38.72^{\prime \prime}$ & $121^{\circ} 18^{\prime} 05.74^{\prime \prime}$ & 2,600 & 650 & - & - & $\mathrm{X}$ & - \\
\hline 9 & $12 \mathrm{~S} / 12 \mathrm{E}-33 \mathrm{ACB}$ & 31050263 & $44^{\circ} 29^{\prime} 23.40^{\prime \prime}$ & $121^{\circ} 17^{\prime} 49.75^{\prime \prime}$ & 1,985 & 740 & $\mathrm{X}$ & $\mathrm{X}$ & - & - \\
\hline 10 & 13S/11E-32DCD & 31000853 & $44^{\circ} 23^{\prime} 40.54^{\prime \prime}$ & $121^{\circ} 26^{\prime} 19.50^{\prime \prime}$ & 2,939 & 620 & $\mathrm{X}$ & - & - & - \\
\hline 11 & 13S/12E-21AAC & 31000654 & $44^{\circ} 26^{\prime} 03.12^{\prime \prime}$ & $121^{\circ} 17^{\prime} 36.77^{\prime \prime}$ & 2,810 & 584 & - & - & $\mathrm{X}$ & - \\
\hline 12 & $13 \mathrm{~S} / 12 \mathrm{E}-22 \mathrm{DAA}$ & 31000829 & $44^{\circ} 25^{\prime} 42.08^{\prime \prime}$ & $121^{\circ} 16^{\prime} 12.50^{\prime \prime}$ & 2,801 & 570 & $\mathrm{X}$ & - & - & - \\
\hline 13 & $13 \mathrm{~S} / 12 \mathrm{E}-26 \mathrm{CCD}$ & 31000830 & $44^{\circ} 24^{\prime} 30.15^{\prime \prime}$ & $121^{\circ} 15^{\prime} 56.42^{\prime \prime}$ & 2,725 & 200 & $X$ & - & - & - \\
\hline 14 & 13S/12E-36BDB & 31000837 & $44^{\circ} 24^{\prime} 08.50^{\prime \prime}$ & $121^{\circ} 14^{\prime} 33.72^{\prime \prime}$ & 2,780 & 460 & $\mathrm{X}$ & $\mathrm{X}$ & - & - \\
\hline 15 & 13S/13E-20DAC & 31000164 & $44^{\circ} 25^{\prime} 26.29^{\prime \prime}$ & $121^{\circ} 11^{\prime} 31.90^{\prime \prime}$ & 2,865 & 540 & $\mathrm{X}$ & - & - & - \\
\hline 16 & 14S/09E-08ABA & 17001804 & $44^{\circ} 22^{\prime} 41.57^{\prime \prime}$ & $121^{\circ} 40^{\prime} 54.84^{\prime \prime}$ & 3,380 & 403 & - & - & $\mathrm{X}$ & - \\
\hline 17 & 14S/10E-23BAA & 17008590 & $44^{\circ} 20^{\prime} 53.00^{\prime \prime}$ & $121^{\circ} 30^{\prime} 13.55^{\prime \prime}$ & 3,310 & 840 & $\mathrm{X}$ & - & - & - \\
\hline 18 & $14 \mathrm{~S} / 10 \mathrm{E}-28 \mathrm{DDC} 2$ & 17009175 & $44^{\circ} 19^{\prime} 16.74^{\prime \prime}$ & $121^{\circ} 32 ' 16.14^{\prime \prime}$ & 3,145 & 149 & - & - & $\mathrm{X}$ & - \\
\hline 19 & $14 \mathrm{~S} / 10 \mathrm{E}-28 \mathrm{DDC} 3$ & 17009174 & $44^{\circ} 19^{\prime} 17.41^{\prime \prime}$ & $121^{\circ} 32^{\prime} 10.39^{\prime \prime}$ & 3,146 & 328 & $\mathrm{X}$ & - & - & - \\
\hline 20 & 14S/10E-30DDB2 & 17001835 & $44^{\circ} 19^{\prime} 26.20^{\prime \prime}$ & $121^{\circ} 34^{\prime} 34.07^{\prime \prime}$ & 3,218 & 200 & - & - & - & $\mathrm{X}$ \\
\hline 21 & 14S/10E-34BCD & 17008523 & $44^{\circ} 18^{\prime} 59.73^{\prime \prime}$ & $121^{\circ} 31^{\prime} 49.11^{\prime \prime}$ & 3,180 & 200 & $\mathrm{X}$ & - & - & - \\
\hline 22 & 14S/11E-01DDD1 & 17001957 & $44^{\circ} 22^{\prime} 43.08^{\prime \prime}$ & $121^{\circ} 21^{\prime} 03.83^{\prime \prime}$ & 2,755 & 501 & - & - & $\mathrm{X}$ & - \\
\hline 23 & 14S/11E-13BCC1 & 17001973 & $44^{\circ} 21^{\prime} 29.63^{\prime \prime}$ & $121^{\circ} 22^{\prime} 13.04^{\prime \prime}$ & 2,798 & 442 & - & - & $\mathrm{X}$ & - \\
\hline 24 & 14S/11E-18BDB & 17009237 & $44^{\circ} 21 ' 37.98^{\prime \prime}$ & $121^{\circ} 27^{\prime} 58.50^{\prime \prime}$ & 3,040 & 560 & $\mathrm{X}$ & - & $\mathrm{X}$ & - \\
\hline 25 & $14 \mathrm{~S} / 11 \mathrm{E}-22 \mathrm{CDB}$ & 17009114 & $44^{\circ} 20^{\prime} 16.22^{\prime \prime}$ & $121^{\circ} 24^{\prime} 19.69^{\prime \prime}$ & 3,100 & 595 & $\mathrm{X}$ & - & - & - \\
\hline 26 & $14 \mathrm{~S} / 12 \mathrm{E}-02 \mathrm{CCC}$ & 17008626 & $44^{\circ} 22^{\prime} 45.20^{\prime \prime}$ & $121^{\circ} 16^{\prime} 04.73^{\prime \prime}$ & 2,680 & 160 & $\mathrm{X}$ & - & - & - \\
\hline 27 & $14 \mathrm{~S} / 12 \mathrm{E}-15 \mathrm{BDC}$ & 17008959 & $44^{\circ} 21^{\prime} 29.62^{\prime \prime}$ & $121^{\circ} 17^{\prime} 04.92^{\prime \prime}$ & 2,603 & 220 & $\mathrm{X}$ & - & - & - \\
\hline 28 & 14S/12E-16DDD & 17009048 & $44^{\circ} 20^{\prime} 59.67^{\prime \prime}$ & $121^{\circ} 17^{\prime} 24.48^{\prime \prime}$ & 2,660 & 180 & $\mathrm{X}$ & - & $\mathrm{X}$ & - \\
\hline 29 & 14S/13E-05BCC & 17008568 & $44^{\circ} 23^{\prime} 11.24^{\prime \prime}$ & $121^{\circ} 12^{\prime} 21.01^{\prime \prime}$ & 2,779 & 220 & $\mathrm{X}$ & - & - & - \\
\hline 30 & 14S/13E-06BBD & 17009049 & $44^{\circ} 23$ '23.11" & $121^{\circ} 13^{\prime} 23.92^{\prime \prime}$ & 2,743 & 200 & $\mathrm{X}$ & - & - & - \\
\hline 31 & 14S/13E-13ACC & 17009283 & $44^{\circ} 21^{\prime} 24.29^{\prime \prime}$ & $121^{\circ} 06^{\prime} 57.72^{\prime \prime}$ & 2,902 & 205 & $\mathrm{X}$ & - & - & - \\
\hline 32 & $14 \mathrm{~S} / 13 \mathrm{E}-22 \mathrm{CAC}$ & 17008542 & $44^{\circ} 20^{\prime} 18.01^{\prime \prime}$ & $121^{\circ} 09^{\prime} 42.51^{\prime \prime}$ & 2,908 & 260 & $\mathrm{X}$ & - & - & - \\
\hline 33 & 14S/13E-27DAA1 & 17000313 & $44^{\circ} 19^{\prime} 34.28^{\prime \prime}$ & $121^{\circ} 08^{\prime} 51.57^{\prime \prime}$ & 2,953 & 252 & - & - & $\mathrm{X}$ & - \\
\hline 34 & 14S/13E-31ABB & 17008574 & $44^{\circ} 19^{\prime} 09.40^{\prime \prime}$ & $121^{\circ} 13^{\prime} 02.70^{\prime \prime}$ & 2,795 & 200 & $\mathrm{X}$ & - & - & - \\
\hline 35 & 14S/13E-35DBD & 17008958 & $44^{\circ} 18^{\prime} 33.93^{\prime \prime}$ & $121^{\circ} 08^{\prime} 00.19^{\prime \prime}$ & 3,002 & 340 & $\mathrm{X}$ & - & - & - \\
\hline 36 & 14S/13E-36CAD & 17009189 & $44^{\circ} 18^{\prime} 36.70^{\prime \prime}$ & $121^{\circ} 07^{\prime} 07.65^{\prime \prime}$ & 2,969 & 300 & $\mathrm{X}$ & - & - & - \\
\hline 37 & 14S/15E-15DAC1 & 13000438 & $44^{\circ} 21^{\prime} 12.57^{\prime \prime}$ & $120^{\circ} 54^{\prime} 28.10^{\prime \prime}$ & 2,910 & 206 & - & - & - & - \\
\hline 38 & $14 \mathrm{~S} / 15 \mathrm{E}-15 \mathrm{DAC} 2$ & 13000909 & $44^{\circ} 21^{\prime} 12.83^{\prime \prime}$ & $120^{\circ} 54^{\prime} 28.01^{\prime \prime}$ & 2,910 & 50 & - & - & - & - \\
\hline 39 & $15 \mathrm{~S} / 10 \mathrm{E}-01 \mathrm{CDD} 2$ & 17000050 & $44^{\circ} 17^{\prime} 30.53^{\prime \prime}$ & $121^{\circ} 29^{\prime} 00.13^{\prime \prime}$ & 3,115 & 320 & - & - & $\mathrm{X}$ & - \\
\hline 40 & 15S/10E-02DCB & 17009187 & $44^{\circ} 17^{\prime} 41.83^{\prime \prime}$ & $121^{\circ} 30^{\prime} 00.73^{\prime \prime}$ & 3,140 & 200 & $\mathrm{X}$ & - & - & - \\
\hline 41 & $15 \mathrm{~S} / 10 \mathrm{E}-05 \mathrm{BBB}$ & 17002999 & $44^{\circ} 18^{\prime} 19.23^{\prime \prime}$ & $121^{\circ} 34^{\prime} 20.56^{\prime \prime}$ & 3,210 & 346 & - & - & - & $\mathrm{X}$ \\
\hline 42 & $15 \mathrm{~S} / 10 \mathrm{E}-10 \mathrm{DBC}$ & 17008625 & $44^{\circ} 16^{\prime} 55.69^{\prime \prime}$ & $121^{\circ} 31^{\prime} 15.98^{\prime \prime}$ & 3,161 & 110 & $\mathrm{X}$ & - & - & - \\
\hline 43 & $15 \mathrm{~S} / 10 \mathrm{E}-25 \mathrm{CAC}$ & 17009190 & $44^{\circ} 14^{\prime} 15.59^{\prime \prime}$ & $121^{\circ} 29^{\prime} 10.99^{\prime \prime}$ & 3,277 & 360 & $\mathrm{X}$ & - & - & - \\
\hline 44 & $15 \mathrm{~S} / 10 \mathrm{E}-25 \mathrm{CCA}$ & 17008614 & $44^{\circ} 14^{\prime} 10.16^{\prime \prime}$ & $121^{\circ} 29^{\prime} 19.33^{\prime \prime}$ & 3,290 & 380 & $\mathrm{X}$ & - & - & - \\
\hline 45 & $15 \mathrm{~S} / 11 \mathrm{E}-04 \mathrm{CDB}$ & 17008569 & $44^{\circ} 17^{\prime} 39.92^{\prime \prime}$ & $121^{\circ} 25^{\prime} 33.26^{\prime \prime}$ & 3,075 & 460 & $\mathrm{X}$ & - & - & - \\
\hline 46 & 15S/11E-06ABD & 17008601 & $44^{\circ} 18^{\prime} 14.22^{\prime \prime}$ & $121^{\circ} 27^{\prime} 27.92^{\prime \prime}$ & 3,140 & 520 & $\mathrm{X}$ & - & - & - \\
\hline 47 & $15 \mathrm{~S} / 11 \mathrm{E}-16 \mathrm{BDB}$ & 17003245 & $44^{\circ} 16^{\prime} 24.18^{\prime \prime}$ & $121^{\circ} 25^{\prime} 33.36^{\prime \prime}$ & 2,935 & 297 & - & - & $\mathrm{X}$ & - \\
\hline 48 & 15S/11E-19AAA & 17008962 & $44^{\circ} 15^{\prime} 41.36^{\prime \prime}$ & $121^{\circ} 27^{\prime} 08.05^{\prime \prime}$ & 3,152 & 360 & $\mathrm{X}$ & - & $\mathrm{X}$ & - \\
\hline 49 & $15 \mathrm{~S} / 12 \mathrm{E}-02 \mathrm{DCA}$ & 17008599 & $44^{\circ} 17^{\prime} 37.29 \prime$ & $121^{\circ} 15^{\prime} 17.29 "$ & 2,915 & 340 & $\mathrm{X}$ & - & - & - \\
\hline 50 & $15 \mathrm{~S} / 12 \mathrm{E}-14 \mathrm{CDD}$ & 17003581 & $44^{\circ} 15^{\prime} 46.63^{\prime \prime}$ & $121^{\circ} 15^{\prime} 39.87^{\prime \prime}$ & 2,975 & 303 & - & - & $\mathrm{X}$ & - \\
\hline
\end{tabular}


Table 1. Data for selected wells in the upper Deschutes Basin, Oregon-Continued

Well location: Refer to "Well- and "Spring-Location System" for explanation. Log-ID number: Identification number permanently assigned to the well by the U.S. Geological Survey. Latitude/Longitude of well in ${ }^{\circ}$, degrees; ', minutes; ”, seconds. Well elevation: Given in feet above sea level. Well depth: Depth of completed well, in feet below land surface. -, no sample or no data collected; X, sample collected.

\begin{tabular}{|c|c|c|c|c|c|c|c|c|c|c|}
\hline $\begin{array}{c}\text { Well } \\
\text { number }\end{array}$ & Well location & $\begin{array}{l}\text { Log-ID } \\
\text { number }\end{array}$ & Latitude & Longitude & $\begin{array}{c}\text { Well } \\
\text { elevation } \\
\text { (feet) }\end{array}$ & $\begin{array}{l}\text { Well } \\
\text { depth } \\
\text { (feet) }\end{array}$ & $\begin{array}{l}\text { Cuttings } \\
\text { samples }\end{array}$ & $\begin{array}{c}\text { Rock } \\
\text { chemistry }\end{array}$ & $\begin{array}{c}\text { Geo- } \\
\text { physical } \\
\text { log }\end{array}$ & $\begin{array}{c}\text { Aquifer } \\
\text { test }\end{array}$ \\
\hline 51 & 15S/13E-03DBD1 & 17003703 & $44^{\circ} 17^{\prime} 46.10^{\prime \prime}$ & $121^{\circ} 09^{\prime} 10.20^{\prime \prime}$ & 3,003 & 320 & - & - & $\mathrm{X}$ & - \\
\hline 52 & $15 \mathrm{~S} / 13 \mathrm{E}-04 \mathrm{CAB} 2$ & 17003723 & $44^{\circ} 17^{\prime} 52.68^{\prime \prime}$ & $121^{\circ} 10^{\prime} 51.42^{\prime \prime}$ & 2,954 & 297 & - & - & $\mathrm{X}$ & - \\
\hline 53 & 15S/13E-07CDD & 17009192 & $44^{\circ} 16^{\prime} 36.09^{\prime \prime}$ & $121^{\circ} 13^{\prime} 15.27^{\prime \prime}$ & 2,974 & 300 & $\mathrm{X}$ & - & - & - \\
\hline 54 & 15S/13E-20AAD & 17051647 & $44^{\circ} 15^{\prime} 32.34^{\prime \prime}$ & $121^{\circ} 11^{\prime} 18.12^{\prime \prime}$ & 2,995 & 802 & $\mathrm{X}$ & $\mathrm{X}$ & $\mathrm{X}$ & - \\
\hline 55 & 15S/13E-21ADB1 & 17003949 & $44^{\circ} 15^{\prime} 25.90^{\prime \prime}$ & $121^{\circ} 10^{\prime} 17.44^{\prime \prime}$ & 3,041 & 390 & - & - & $\mathrm{X}$ & - \\
\hline 56 & $15 \mathrm{~S} / 13 \mathrm{E}-22 \mathrm{BCD}$ & 17003952 & $44^{\circ} 15^{\prime} 18.53^{\prime \prime}$ & $121^{\circ} 09^{\prime} 46.25^{\prime \prime}$ & 3,045 & 740 & - & - & $\mathrm{X}$ & - \\
\hline 57 & $15 \mathrm{~S} / 13 \mathrm{E}-22 \mathrm{CBA} 2$ & 17003951 & $44^{\circ} 15^{\prime} 14.90^{\prime \prime}$ & $121^{\circ} 09^{\prime} 46.47^{\prime \prime}$ & 3,053 & 801 & - & - & - & $\mathrm{X}$ \\
\hline 58 & 15S/13E-29CAD2 & 17003981 & $44^{\circ} 14^{\prime} 14.15^{\prime \prime}$ & $121^{\circ} 11^{\prime} 57.60^{\prime \prime}$ & 3,090 & 485 & - & - & $\mathrm{X}$ & - \\
\hline 59 & 15S/13E-31DAA & 17009103 & $44^{\circ} 13 ’ 31.61^{\prime \prime}$ & $121^{\circ} 12^{\prime} 35.99^{\prime \prime}$ & 3,118 & 475 & $\mathrm{X}$ & - & - & - \\
\hline 60 & 15S/14E-03BAA & 13002959 & $44^{\circ} 18^{\prime} 19.43^{\prime \prime}$ & $121^{\circ} 02^{\prime} 20.65^{\prime \prime}$ & 2,991 & 320 & $\mathrm{X}$ & $\mathrm{X}$ & - & - \\
\hline 61 & $15 \mathrm{~S} / 14 \mathrm{E}-14 \mathrm{CDC}$ & 13002960 & $44^{\circ} 15^{\prime} 47.85^{\prime \prime}$ & $121^{\circ} 01^{\prime} 13.39^{\prime \prime}$ & 3,060 & 400 & $X$ & $\mathrm{X}$ & - & - \\
\hline 62 & $15 \mathrm{~S} / 14 \mathrm{E}-28 \mathrm{DCD}$ & 13002967 & $44^{\circ} 14^{\prime} 01.43^{\prime \prime}$ & $121^{\circ} 03,13.06 "$ & 3,104 & 450 & $\mathrm{X}$ & - & - & - \\
\hline 63 & 15S/15E-01BDA & 13000498 & $44^{\circ} 18^{\prime} 00.64^{\prime \prime}$ & $120^{\circ} 52^{\prime} 37.73^{\prime \prime}$ & 3,241 & 1,000 & - & - & $\mathrm{X}$ & - \\
\hline 64 & $15 \mathrm{~S} / 15 \mathrm{E}-23 \mathrm{BBB}$ & 13003200 & $44^{\circ} 15^{\prime} 40.68^{\prime \prime}$ & $120^{\circ} 54^{\prime} 18.92^{\prime \prime}$ & 3,265 & 410 & $\mathrm{X}$ & $\mathrm{X}$ & - & - \\
\hline 65 & 15S/15E-29BAA & 13003063 & $44^{\circ} 14^{\prime} 49.26^{\prime \prime}$ & $120^{\circ} 57^{\prime} 25.65^{\prime \prime}$ & 3,210 & 500 & $\mathrm{X}$ & - & - & - \\
\hline 66 & 16S/10E-13BBB2 & 17009825 & $44^{\circ} 11^{\prime} 42.77^{\prime \prime}$ & $121^{\circ} 28^{\prime} 55.57^{\prime \prime}$ & 3,375 & 320 & $\mathrm{X}$ & $\mathrm{X}$ & - & - \\
\hline 67 & 16S/11E-09BAC & 17009069 & $44^{\circ} 12^{\prime} 28.25^{\prime \prime}$ & $121^{\circ} 24^{\prime} 58.07^{\prime \prime}$ & 3,221 & 360 & $\mathrm{X}$ & - & - & - \\
\hline 68 & $16 \mathrm{~S} / 11 \mathrm{E}-12 \mathrm{ADB}$ & 17009059 & $44^{\circ} 12^{\prime} 21.04^{\prime \prime}$ & $121^{\circ} 20^{\prime} 47.69^{\prime \prime}$ & 3,281 & 650 & $\mathrm{X}$ & - & - & - \\
\hline 69 & $16 \mathrm{~S} / 11 \mathrm{E}-12 \mathrm{ADB}$ & 17008638 & $44^{\circ} 12 ' 10.46^{\prime \prime}$ & $121^{\circ} 21^{\prime} 29.43^{\prime \prime}$ & 3,281 & 675 & $\mathrm{X}$ & - & - & - \\
\hline 70 & 16S/11E-18BAC & 17009240 & $44^{\circ} 11^{\prime} 38.13^{\prime \prime}$ & $121^{\circ} 27^{\prime} 23.39 ”$ & 3,319 & 392 & $\mathrm{X}$ & $\mathrm{X}$ & - & - \\
\hline 71 & 16S/11E-33DDD & 17009104 & $44^{\circ} 08^{\prime} 19.07^{\prime \prime}$ & $121^{\circ} 24^{\prime} 13.60^{\prime \prime}$ & 3,522 & 750 & $\mathrm{X}$ & $\mathrm{X}$ & - & - \\
\hline 72 & $16 \mathrm{~S} / 11 \mathrm{E}-35 \mathrm{ACA} 2$ & 17008615 & $44^{\circ} 08^{\prime} 55.49^{\prime \prime}$ & $121^{\circ} 22^{\prime} 08.94^{\prime \prime}$ & 3,410 & 708 & $\mathrm{X}$ & - & - & - \\
\hline 73 & 16S/12E-10DCC & 17009081 & $44^{\circ} 11^{\prime} 49.83^{\prime \prime}$ & $121^{\circ} 16^{\prime} 20.20^{\prime \prime}$ & 3,215 & 528 & $\mathrm{X}$ & - & - & - \\
\hline 74 & $16 \mathrm{~S} / 12 \mathrm{E}-13 \mathrm{BBB}$ & 17008524 & $44^{\circ} 11^{\prime} 41.35^{\prime \prime}$ & $121^{\circ} 14^{\prime} 26.64^{\prime \prime}$ & 3,185 & 540 & $\mathrm{X}$ & - & - & - \\
\hline 75 & $16 \mathrm{~S} / 12 \mathrm{E}-14 \mathrm{ACA}$ & 17009101 & $44^{\circ} 11^{\prime} 27.83^{\prime \prime}$ & $121^{\circ} 14^{\prime} 55.56^{\prime \prime}$ & 3,185 & 580 & $\mathrm{X}$ & - & - & - \\
\hline 76 & 16S/12E-14BCC & 17008612 & $44^{\circ} 11^{\prime} 23.69^{\prime \prime}$ & $121^{\circ} 15^{\prime} 36.93^{\prime \prime}$ & 3,185 & 430 & $\mathrm{X}$ & - & - & - \\
\hline 77 & $16 \mathrm{~S} / 12 \mathrm{E}-17 \mathrm{BBD}$ & 17008526 & $44^{\circ} 11^{\prime} 35.15^{\prime \prime}$ & $121^{\circ} 19^{\prime} 10.35^{\prime \prime}$ & 3,210 & 568 & $\mathrm{X}$ & - & - & - \\
\hline 78 & $16 \mathrm{~S} / 12 \mathrm{E}-19 \mathrm{ABA}$ & 17008636 & $44^{\circ} 10^{\prime} 49.03 "$ & $121^{\circ} 19^{\prime} 43.05^{\prime \prime}$ & 3,265 & 600 & $\mathrm{X}$ & - & - & - \\
\hline 79 & 16S/12E-21BDB & 17008637 & $44^{\circ} 10^{\prime} 41.17^{\prime \prime}$ & $121^{\circ} 17^{\prime} 50.28^{\prime \prime}$ & 3,270 & 600 & $\mathrm{X}$ & - & - & - \\
\hline 80 & $16 \mathrm{~S} / 12 \mathrm{E}-30 \mathrm{BAA}$ & 17009051 & $44^{\circ} 09^{\prime} 59.43^{\prime \prime}$ & $121^{\circ} 20^{\prime} 00.13^{\prime \prime}$ & 3,285 & 625 & $\mathrm{X}$ & - & - & - \\
\hline 81 & 16S/13E-16AAC & 17004656 & $44^{\circ} 11^{\prime} 33.28^{\prime \prime}$ & $121^{\circ} 10^{\prime} 01.08^{\prime \prime}$ & 3,164 & 505 & - & - & $\mathrm{X}$ & - \\
\hline 82 & 16S/13E-16CCA & 17010139 & $44^{\circ} 10^{\prime} 59.92^{\prime \prime}$ & $121^{\circ} 10^{\prime} 04.12^{\prime \prime}$ & 3,215 & 580 & $\mathrm{X}$ & - & - & - \\
\hline 83 & $16 \mathrm{~S} / 14 \mathrm{E}-17 \mathrm{CDD}$ & 13050194 & $44^{\circ} 10^{\prime} 51.81^{\prime \prime}$ & $121^{\circ} 04^{\prime} 35.20^{\prime \prime}$ & 3,160 & 482 & $\mathrm{X}$ & $\mathrm{X}$ & - & - \\
\hline 84 & 16S/14E-21BBD & 13002966 & $44^{\circ} 10^{\prime} 41.16^{\prime \prime}$ & $121^{\circ} 03^{\prime} 40.04^{\prime \prime}$ & 3,241 & 640 & $\mathrm{X}$ & $\mathrm{X}$ & - & - \\
\hline 85 & 17S/11E-03BBC1 & 17004676 & $44^{\circ} 08^{\prime} 09.07^{\prime \prime}$ & $121^{\circ} 24^{\prime} 05.59^{\prime \prime}$ & 3,538 & 670 & - & - & $\mathrm{X}$ & - \\
\hline 86 & 17S/11E-24DCA & 17001490 & $44^{\circ} 05^{\prime} 02.96^{\prime \prime}$ & $121^{\circ} 20^{\prime} 58.36^{\prime \prime}$ & 3,665 & 990 & $X$ & $\mathrm{X}$ & - & - \\
\hline 87 & 17S/11E-25CBA1 & 17004713 & $44^{\circ} 04^{\prime} 22.60^{\prime \prime}$ & $121^{\circ} 21^{\prime} 36.31^{\prime \prime}$ & 3,745 & 358 & - & - & $\mathrm{X}$ & - \\
\hline 88 & 17S/12E-09CCD1 & 17004896 & $44^{\circ} 06^{\prime} 35.92^{\prime \prime}$ & $121^{\circ} 17^{\prime} 57.55^{\prime \prime}$ & 3,465 & 454 & - & - & $\mathrm{X}$ & - \\
\hline 89 & $17 \mathrm{~S} / 12 \mathrm{E}-11 \mathrm{DDB}$ & 17009078 & $44^{\circ} 06^{\prime} 42.09^{\prime \prime}$ & $121^{\circ} 14^{\prime} 43.43^{\prime \prime}$ & 3,400 & 640 & $\mathrm{X}$ & $\mathrm{X}$ & - & - \\
\hline 90 & 17S/13E-19ABD & 17005118 & $44^{\circ} 05^{\prime} 34.22^{\prime \prime}$ & $121^{\circ} 12^{\prime} 33.56^{\prime \prime}$ & 3,462 & 627 & - & - & $X$ & - \\
\hline 91 & 17S/13E-20DAD & 17009161 & $44^{\circ} 05^{\prime} 08.10^{\prime \prime}$ & $121^{\circ} 11^{\prime} 03.68^{\prime \prime}$ & 3,415 & 625 & $\mathrm{X}$ & $\mathrm{X}$ & - & - \\
\hline 92 & 17S/14E-02CAA & 17009317 & $44^{\circ} 07^{\prime} 35.39^{\prime \prime}$ & $121^{\circ} 01^{\prime} 05.95^{\prime \prime}$ & 3,306 & 845 & $\mathrm{X}$ & $\mathrm{X}$ & $\mathrm{X}$ & - \\
\hline 93 & 17S/14E-11DCB & 17009319 & $44^{\circ} 06^{\prime} 29.49^{\prime \prime}$ & $121^{\circ} 00^{\prime} 57.96^{\prime \prime}$ & 3,350 & 720 & $\mathrm{X}$ & $\mathrm{X}$ & - & - \\
\hline 94 & $17 \mathrm{~S} / 14 \mathrm{E}-28 \mathrm{ABC}$ & 17001610 & $44^{\circ} 04^{\prime} 34.87^{\prime \prime}$ & $121^{\circ} 03,14.81^{\prime \prime}$ & 3,365 & 680 & $\mathrm{X}$ & - & - & - \\
\hline 95 & 18S/09E-20BDA & 17005215 & $44^{\circ} 00^{\prime} 16.82^{\prime \prime}$ & $121^{\circ} 03 ’ 14.81^{\prime \prime}$ & 6,310 & 780 & - & - & - & $\mathrm{X}$ \\
\hline 96 & 18S/11E-21CDD & 17005258 & $43^{\circ} 59^{\prime} 37.48^{\prime \prime}$ & $121^{\circ} 24^{\prime} 50.05^{\prime \prime}$ & 4,060 & 547 & - & - & $\mathrm{X}$ & - \\
\hline 97 & $18 \mathrm{~S} / 11 \mathrm{E}-27 \mathrm{ACD}$ & 17009163 & $43^{\circ} 59^{\prime} 14.55^{\prime \prime}$ & $121^{\circ} 23^{\prime} 22.70^{\prime \prime}$ & 3,960 & 205 & $\mathrm{X}$ & - & - & - \\
\hline 98 & $18 \mathrm{~S} / 11 \mathrm{E}-27 \mathrm{BCB} 1$ & 17005526 & $43^{\circ} 59^{\prime} 20.96^{\prime \prime}$ & $121^{\circ} 24^{\prime} 06.37^{\prime \prime}$ & 3,942 & 300 & - & - & $\mathrm{X}$ & - \\
\hline 99 & $18 \mathrm{~S} / 12 \mathrm{E}-05 \mathrm{BDB}$ & 17005576 & $44^{\circ} 02^{\prime} 47.39^{\prime \prime}$ & $121^{\circ} 19^{\prime} 01.41^{\prime \prime}$ & 3,620 & 701 & - & - & $\mathrm{X}$ & $\mathrm{X}$ \\
\hline 100 & $18 \mathrm{~S} / 12 \mathrm{E}-05 \mathrm{CCC} 1$ & 17005584 & $44^{\circ} 02^{\prime} 15.71^{\prime \prime}$ & $121^{\circ} 19^{\prime} 20.20^{\prime \prime}$ & 3,633 & 258 & - & - & $\mathrm{X}$ & - \\
\hline
\end{tabular}


Table 1. Data for selected wells in the upper Deschutes Basin, Oregon-Continued

Well location: Refer to "Well- and "Spring-Location System" for explanation. Log-ID number: Identification number permanently assigned to the well by the U.S. Geological Survey. Latitude/Longitude of well in ${ }^{\circ}$, degrees; ', minutes; ”, seconds. Well elevation: Given in feet above sea level. Well depth: Depth of completed well, in feet below land surface. - , no sample or no data collected; X, sample collected.

\begin{tabular}{|c|c|c|c|c|c|c|c|c|c|c|}
\hline $\begin{array}{c}\text { Well } \\
\text { number }\end{array}$ & Well location & $\begin{array}{l}\text { Log-ID } \\
\text { number }\end{array}$ & Latitude & Longitude & $\begin{array}{c}\text { Well } \\
\text { elevation } \\
\text { (feet) }\end{array}$ & $\begin{array}{l}\text { Well } \\
\text { depth } \\
\text { (feet) }\end{array}$ & $\begin{array}{l}\text { Cuttings } \\
\text { samples }\end{array}$ & $\begin{array}{c}\text { Rock } \\
\text { chemistry }\end{array}$ & $\begin{array}{c}\text { Geo- } \\
\text { physical } \\
\text { log }\end{array}$ & $\begin{array}{c}\text { Aquifer } \\
\text { test }\end{array}$ \\
\hline 101 & 18S/12E-07DBD1 & 17001738 & $44^{\circ} 01^{\prime} 40.99^{\prime \prime}$ & $121^{\circ} 19^{\prime} 44.07^{\prime \prime}$ & 3,835 & 800 & $\mathrm{X}$ & $\mathrm{X}$ & - & - \\
\hline 102 & 18S/12E-10ADA1 & 17005597 & $44^{\circ} 01^{\prime} 55.82^{\prime \prime}$ & $121^{\circ} 15^{\prime} 52.63^{\prime \prime}$ & 3,688 & 750 & - & - & $\mathrm{X}$ & - \\
\hline 103 & 18S/12E-14CAD & 17009576 & $44^{\circ} 00^{\prime} 42.73^{\prime \prime}$ & $121^{\circ} 15^{\prime} 10.16^{\prime \prime}$ & 3,698 & 715 & $\mathrm{X}$ & - & $\mathrm{X}$ & - \\
\hline 104 & 18S/12E-14DBD & 17009577 & $44^{\circ} 00^{\prime} 45.83^{\prime \prime}$ & $121^{\circ} 14^{\prime} 56.50^{\prime \prime}$ & 3,703 & 760 & $\mathrm{X}$ & - & $X$ & - \\
\hline 105 & $18 \mathrm{~S} / 12 \mathrm{E}-31 \mathrm{DCA}$ & 17009399 & $43^{\circ} 58^{\prime} 01.98^{\prime \prime}$ & $121^{\circ} 19^{\prime} 41.94 "$ & 4,125 & 670 & $\mathrm{X}$ & - & - & - \\
\hline 106 & 18S/13E-01CCD & 17005701 & $44^{\circ} 02^{\prime} 14.49^{\prime \prime}$ & $121^{\circ} 07^{\prime} 08.54^{\prime \prime}$ & 3,495 & 902 & - & - & $X$ & - \\
\hline 107 & $18 \mathrm{~S} / 13 \mathrm{E}-10 \mathrm{AAC}$ & 17005709 & $44^{\circ} 02^{\prime} 00.87^{\prime \prime}$ & $121^{\circ} 08^{\prime} 49.11^{\prime \prime}$ & 3,570 & 875 & - & - & $X$ & - \\
\hline 108 & 18S/13E-24DCB & 17009222 & $43^{\circ} 59^{\prime} 43.90^{\prime \prime}$ & $121^{\circ} 06 ' 58.41^{\prime \prime}$ & 3,612 & 710 & $\mathrm{X}$ & $\mathrm{X}$ & 一 & - \\
\hline 109 & 19S/11E-16ACC & 17005741 & $43^{\circ} 55^{\prime} 48.04^{\prime \prime}$ & $121^{\circ} 24^{\prime} 48.39^{\prime \prime}$ & 4,187 & 247 & - & - & $\mathrm{X}$ & - \\
\hline 110 & 19S/11E-25BAA & 17050111 & $43^{\circ} 54^{\prime} 22.79^{\prime \prime}$ & $121^{\circ} 21^{\prime} 21.13$ ' & 4,515 & 434 & - & - & $\mathrm{X}$ & - \\
\hline 111 & 19S/14E-02DAA2 & 17009126 & $43^{\circ} 57^{\prime} 07.94 \prime$ & $121^{\circ} 00^{\prime} 27.71^{\prime \prime}$ & 3,579 & 1,135 & $X$ & - & - & - \\
\hline 112 & $20 \mathrm{~S} / 10 \mathrm{E}-12 \mathrm{CCC}$ & 17008629 & $43^{\circ} 51^{\prime} 00.33^{\prime \prime}$ & $121^{\circ} 29^{\prime} 05.35^{\prime \prime}$ & 4,221 & 102 & $X$ & - & - & - \\
\hline 113 & 21S/10E-21BDC & 17008560 & $43^{\circ} 44^{\prime} 26.62^{\prime \prime}$ & $121^{\circ} 32^{\prime} 20.54^{\prime \prime}$ & 4,257 & 80 & $\mathrm{X}$ & - & - & - \\
\hline 114 & $21 \mathrm{~S} / 11 \mathrm{E}-18 \mathrm{CDA} 3$ & 17007618 & $43^{\circ} 45^{\prime} 01.88^{\prime \prime}$ & $121^{\circ} 27^{\prime} 24.05^{\prime \prime}$ & 4,208 & 40 & - & - & $\mathrm{X}$ & - \\
\hline 115 & 21S/11E-32BBD & 17008562 & $43^{\circ} 42^{\prime} 53.17^{\prime \prime}$ & $121^{\circ} 26^{\prime} 34.89^{\prime \prime}$ & 4,265 & 115 & $X$ & - & - & - \\
\hline 116 & 22S/10E-08DBD & 17008561 & $43^{\circ} 40^{\prime} 44.65^{\prime \prime}$ & $121^{\circ} 33^{\prime} 04.14^{\prime \prime}$ & 4,237 & 40 & $X$ & - & - & - \\
\hline 117 & $22 \mathrm{~S} / 10 \mathrm{E}-14 \mathrm{CBB}$ & 17008506 & $43^{\circ} 39^{\prime} 55.73^{\prime \prime}$ & $121^{\circ} 30^{\prime} 10.49$ '” & 4,228 & 27 & $X$ & - & - & - \\
\hline 118 & $22 \mathrm{~S} / 10 \mathrm{E}-14 \mathrm{CCA}$ & 17009173 & $43^{\circ} 39^{\prime} 47.96^{\prime \prime}$ & $121^{\circ} 30^{\prime} 00.83^{\prime \prime}$ & 4,227 & 555 & $X$ & - & - & - \\
\hline 119 & 23S/09E-36BBC & 35000136 & $43^{\circ} 32^{\prime} 31.81^{\prime \prime}$ & $121^{\circ} 36^{\prime} 03.10^{\prime \prime}$ & 4,332 & 470 & - & - & $\mathrm{X}$ & - \\
\hline 120 & $23 \mathrm{~S} / 10 \mathrm{E}-36 \mathrm{DDC}$ & 35000138 & $43^{\circ} 31^{\prime} 52.61^{\prime \prime}$ & $121^{\circ} 28^{\prime} 13.86^{\prime \prime}$ & 4,308 & 130 & - & - & $\mathrm{X}$ & - \\
\hline
\end{tabular}

Fifty samples of lava from cuttings collected from wells within the project area were selected for chemical analysis. The samples were analyzed with an X-ray fluorescence technique at the Washington State University GeoAnalytical Laboratory. The analysis technique used is described in Johnson and others (1999).

\section{GEOLOGIC SETTING}

\section{Regional Setting}

Most of the upper Deschutes Basin falls within two major geologic provinces (fig. 5), the Cascade Range and the Basin and Range Province (Baldwin, 1981). The Basin and Range Province is considered herein to include the southern part of the High Lava Plains. The geologic processes that have operated in these provinces have overlapped and interacted in much of the upper Deschutes Basin. The Cascade Range is a north-south trending zone of compositionally diverse volcanic eruptive centers with deposits extending from northern California to southern British Columbia. Prominent among the eruptive centers in the Deschutes Basin are large stratovolcanoes such as North, Middle, and South Sister, and Mount Jefferson, all of which exceed 10,000 ft above sea level in elevation. The Cascade Range is primarily a constructional feature, but its growth has been accompanied, at least in places, by subsidence of the range into a north-south trending graben (Allen, 1966). Green Ridge is the eastern escarpment of one of the graben-bounding faults. The Basin and Range Province is a region of crustal extension and is characterized by subparallel fault-bounded down-dropped basins separated by fault-block ranges.

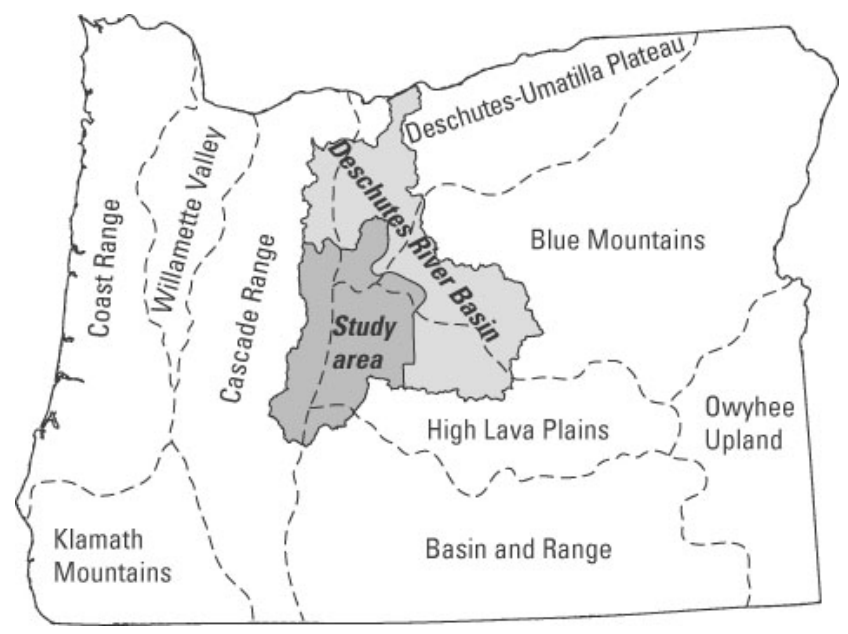

Figure 5. Location of the Deschutes Basin in relation to the physiographic provinces of Oregon. (Provinces from Baldwin, 1981) 
Individual basins and intervening ranges are typically 10 to 20 miles across. The Basin and Range Province encompasses much of the interior of the Western United States, extending from central Oregon south through Nevada and western Utah, into the southern parts of California, Arizona, and New Mexico. Although the Basin and Range Province is primarily structural, faulting has been accompanied by widespread volcanism.

\section{Depositional History and Mechanisms}

Most of the geologic features in the study area are the result of about 30 million years of volcanic activity related to a north-south trending volcanic arc, the most recent manifestation of which are today's Cascade Range volcanoes. Volcanic and tectonic activity over the past 8 to 10 million years in the Basin and Range and High Lava Plains has also helped shape the present landscape. Most of the rock units in the study area that are important water-bearing units were formed within the past 7 million years.

Various types of volcanic rocks and associated sedimentary deposits have different hydrologic properties, such as permeability. Understanding the volcanic and depositional processes that control the distribution of different rock types, therefore, can provide insight into the geographic distribution of permeability. The hypothetical cross-section in figure 6 illustrates some of those concepts. For example, the proportion of lava decreases, and the proportion of volcanically derived sediment increases, with distance from a volcanic vent. This factor influences distribution of permeability within the Deschutes Basin. In addition, the grain size of volcaniclastic sediment tends to decrease as distance from the volcanic vent increases. This also has a direct bearing on permeability. Some volcanic products, such as gas-charged ash-flows (ignimbrites) and some basalt flows, have enough fluidity to travel miles from their sources, often along preexisting stream drainages. These drainagefilling deposits create variations in permeability, and the paleodrainages may define preferential flow paths within the ground-water flow system. Finally, at some distance away from volcanic vents, fluvial processes dominate volcanic processes and become the major factor controlling grain-size distribution.

The oldest rock unit within the study area, the John Day Formation, is about 20 to 40 million years old. The John Day Formation comprises several thousand feet of diagenetically altered volcanic and volcanically derived sedimentary deposits (Robinson and others, 1984). The unit also contains numerous partially eroded volcanic vents. Powell Buttes, Smith Rock, and Juniper Butte are examples of John Day Formation outcrops within the study area.

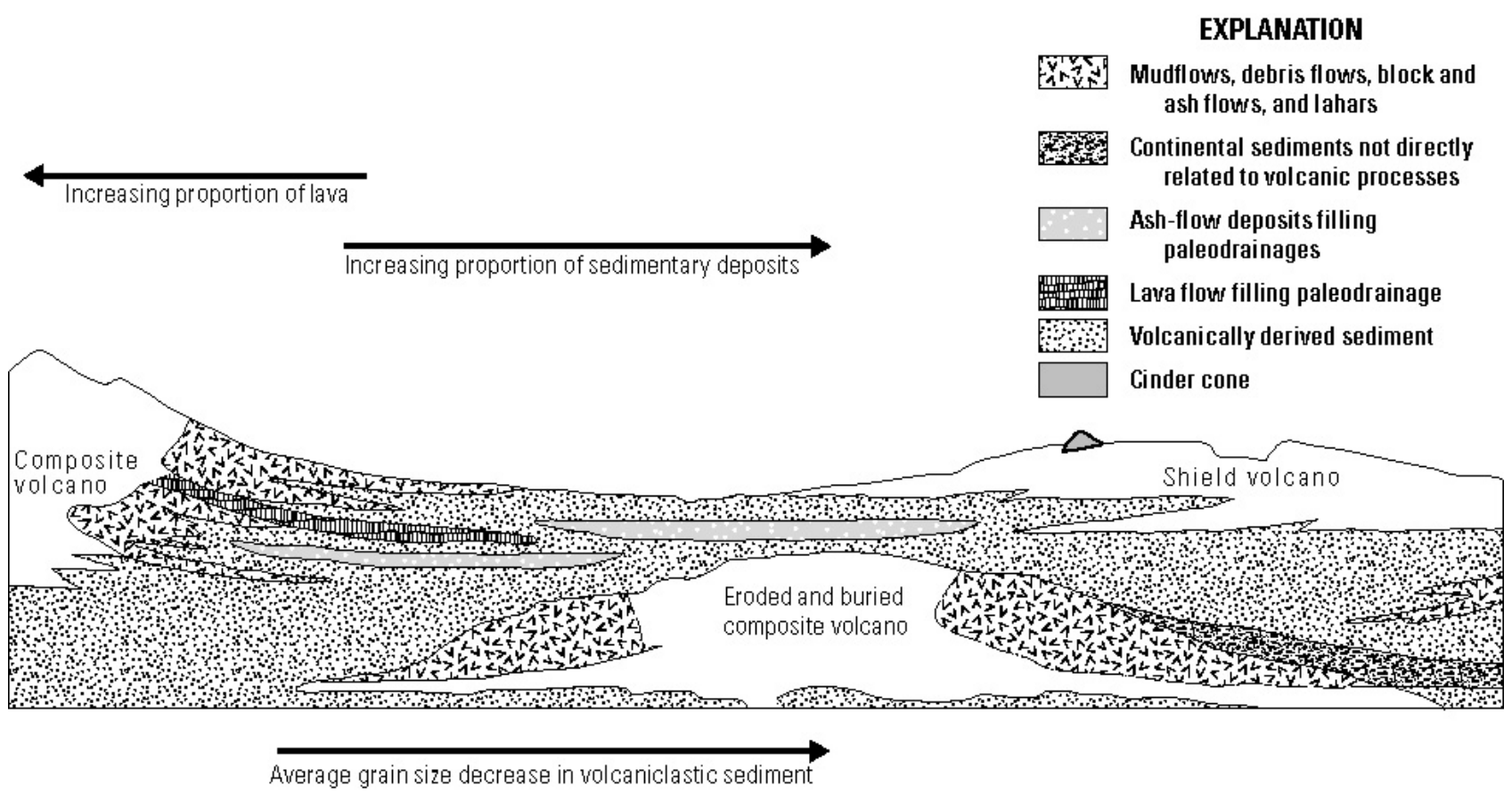

Figure 6. Diagrammatic section through a volcanic arc and adjacent area showing the relation between volcanic and sedimentary facies. (Modified from Sherrod and Smith, 2000) 
The next major surge in volcanic activity occurred between 7 and 4 million years ago. That volcanic activity produced the Deschutes Formation, a sequence of lava flows, pyroclastic deposits, volcaniclastic deposits, and sediment more than 2,000 ft thick (Smith, 1986b). The volcanic eruptions that generated the Deschutes Formation culminated with the formation of a down-faulted depression along the axis of the Cascade Range (Allen, 1966; Smith and others, 1987). The western slope of Green Ridge, along the Metolius River, is an escarpment formed by one of the faults bounding the depression. Subsequent volcanic deposits have buried most other bounding faults.

Other volcanic activity was occurring within the High Lava Plains and Basin and Range Provinces coincident with that associated with the Deschutes Formation. Lava flows of today's Horse Ridge and Walker Rim are notable products of that activity.

The last episode of volcanic activity has been ongoing since the late Pliocene, or about the past 3 million years (Sherrod and Smith, 2000). The focus of that activity has been predominately within the Cascade Range and at Newberry Volcano. Volcanic activity in the Cascade Range has produced a few thousand feet of lava flows with lesser pyroclastic material and sediment. Thicker accumulations occur at the sites of the large composite volcanoes such as North, Middle, and South Sister. The youngest volcanic deposit within the study area, an obsidian flow and related pyroclastic deposit at Newberry Volcano, is about 1,300 years old (MacLeod and others, 1995).

Glacial deposits within the study area result from episodic glaciation since the late Pleistocene, a period of about 150,000 years (Scott, 1977; Scott and Gardner, 1992; Sherrod and others, in press). However, most of the glacial material mapped within the study area is from the Suttle Lake advance (of the Cabot Creek glaciation), which culminated about 20,000 years ago (Sherrod and others, in press). Minor Neoglacial deposits, which occur near the summits of the highest peaks, range in age from a few hundred to a few thousand years.

\section{STRATIGRAPHIC UNITS}

\section{John Day Formation}

The John Day Formation, the oldest unit in the study area, consists of a stratigraphically complex assemblage of early Tertiary lava flows, pyroclastic deposits, sedimentary strata, and volcanic vent deposits (unit Tjd on plate 1) (Robinson and others, 1984; Smith and others, 1998). The unit ranges in age from 39.17 Ma (mega-annum: million years before present) to $22 \mathrm{Ma}$ (Smith and others, 1998). The John Day Formation is exposed in outcrops along the eastern and northern edges of the study area. The unit as a whole has relatively low permeability and is considered to be the hydrologic basement for the ground-water flow system within the study area (Gannett and others, 2001).

\section{Prineville Basalt}

Middle Miocene volcanic activity near presentday Bowman Dam produced a few hundred feet of lava flows called the Prineville Basalt (Uppuluri, 1974); its age is about 15.7 Ma (Smith, 1986b). The Prineville Basalt (unit Tpb on plate 1) crops out in the Crooked River canyon near Prineville, the Deschutes River canyon north of Round Butte Dam, and near Gateway. Locally it serves as a source of ground water in the area between the Crooked River and the northern side of Powell Buttes (east of Dry River), and near Madras and Gateway.

\section{Deschutes Formation}

Late Tertiary Deschutes Formation and ageequivalent strata constitute the principal aquifer within the study area. The Deschutes Formation ranges in age from 7.5 to 4.0 Ma (Smith, 1986b). The rock units are identified on plate 1. The Deschutes Formation comprises hundreds of volcanic deposits and countless sedimentary beds.

Deschutes Formation material represents a classic example of basin-filling sedimentation resulting from episodic volcanic eruptions. The volcanic and volcaniclastic depositional mechanisms discussed in the previous section apply to the Deschutes Formation.

The Deschutes Formation includes several intrabasin volcanic vents, which range from basalt to rhyolite in composition. Notable mafic vents include Tetherow, Long, and Awbrey Buttes, whereas silicic vents include a rhyolite dome complex at Cline Buttes and a rhyodacite dome complex near Steelhead Falls. The silicic domes exhibit remarkably high permeability. 
Smith (1986b) recognized three major depositional environments in the Deschutes Formation: an arc-adjacent alluvial plain, the ancestral Deschutes River, and the inactive-basin margin. These environments are responsible for the compositional variability within the formation (fig. 7). The arc-adjacent alluvial plain comprises Cascade Range derived lava flows, ash-flow tuffs, fallout tephra, volcaniclastic sediment, debris flows, and hyperconcentrated flood deposits. The ancestral Deschutes River deposits include alluvial-channel deposits, hyperconcentrated flood deposits, and intracanyon lava flows and ash-flow deposits. The inactive-basin margin comprises poorly sorted alluvium originating from the Ochoco and Mutton Mountains, reworked fallout tephra from the Cascade Range, and sparse lava flows from vents south and east of the basin.

The permeability of the Deschutes Formation and equivalent rocks ranges from relatively low in finegrained sedimentary deposits, dense lava flows, and ignimbrites to high in coarse-grained unconsolidated sedimentary deposits and vesicular and brecciated lava flows. Two basalt units within the Deschutes Formation, the Opal Springs basalt and Pelton basalt, are well known for their large springs that discharge to the Crooked and Deschutes Rivers (Stearns, 1931; Sceva, 1968).

\section{Deposits of the Cascade Range and Newberry Volcano}

The Cascade Range and parts of the High Lava Plains and Basin and Range Provinces consist of late Tertiary to Quaternary lava flows, lava domes, volcanic vents, pyroclastic deposits, and volcaniclastic sediment (Sherrod and Smith, 2000). Newberry Volcano is composed of similar material but is all Quaternary in age. The rock units are identified on plate 1 . The relatively young volcanic deposits are thick in the Cascade Range, where 1.8-Ma rocks were found at a depth of 3,044 ft at Santiam Pass (Hill, 1992).

The surfaces of the young volcanic deposits are commonly fractured and brecciated, making them extremely permeable. Much of the precipitation in the Cascade Range percolates through these rocks to recharge the ground-water flow system. The large number of springs and lack of incised surface-water drainages in the Cascade Range, high seepage loss from canals near Bend, and high streamflow loss south of Bend demonstrate the permeable nature of the deposits (Gannett and others, 2001).

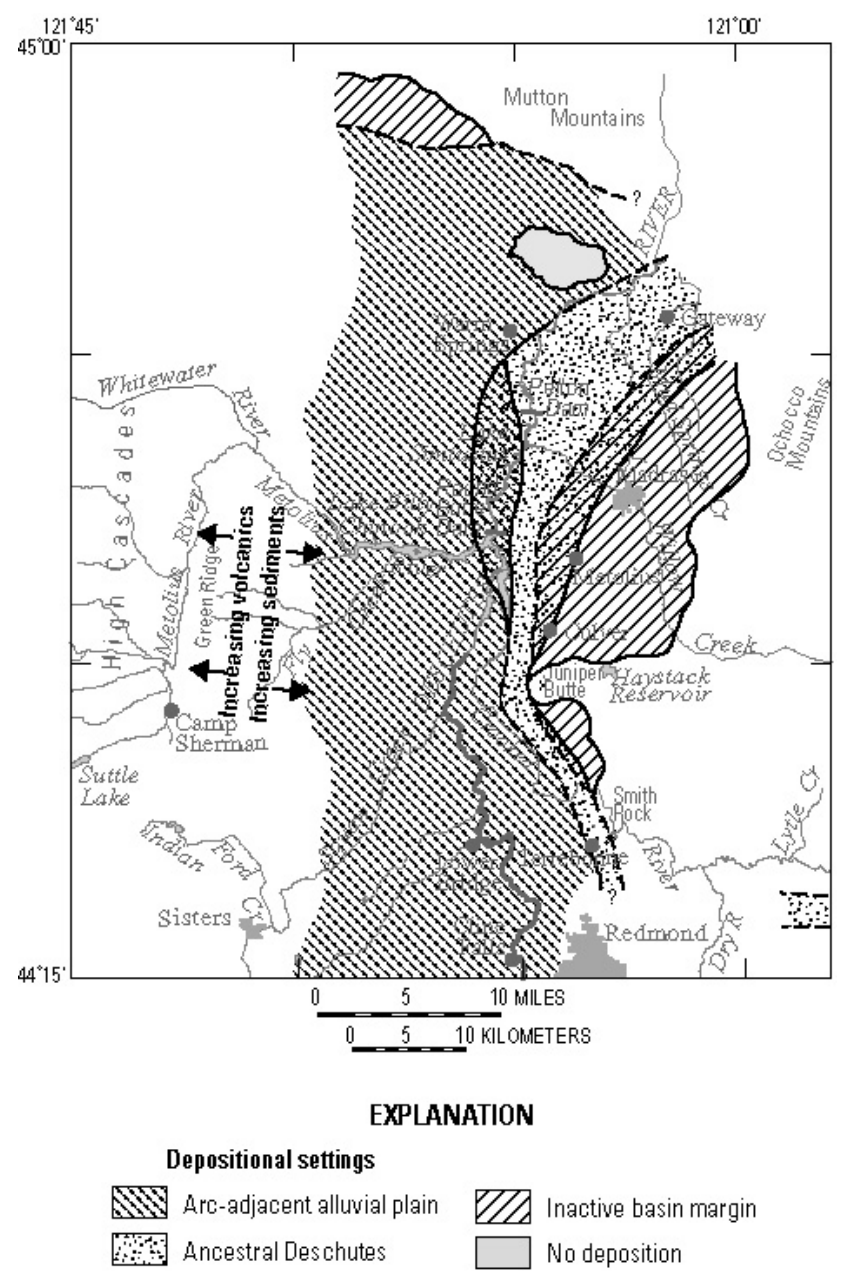

Figure 7. Areal distribution of facies within the Deschutes Formation. (From Smith, 1991)

\section{Quaternary Sedimentary Deposits}

Several Quaternary-age sedimentary deposits occur within the study area (plate 1). Pleistocene to Holocene sedimentary deposits (Qs) resulted from several processes, including mass wasting of upland deposits, alluviation by high-energy streams, and deposition in low-energy streams and lakes. These sedimentary deposits are locally a few hundred feet thick. Glacial deposits $(\mathrm{Qg})$ are predominately Pleistoceneage till. Glacial deposits are generally less than a few tens of feet thick except at lateral moraines, where deposits may be as much as a few hundred feet thick. Alluvial deposits (Qal) are mostly late Pleistocene to Holocene in age and are generally less than a few tens of feet thick. Alluvial deposits have not been distinguished from the generalized sedimentary unit (Qs) on some of the published geologic maps, particularly within the La Pine subbasin. 


\section{TECTONIC STRUCTURES}

Faults and fault-bounded grabens are the most common geologic structures in the upper Deschutes Basin. Faults can have a profound effect on ground water, serving either as boundaries to flow or as conduits for flow. Faults can act as boundaries to flow where they juxtapose rocks of contrasting permeability. This juxtaposition can be caused by fault movement, or by the fault's influence on subsequent deposition. In the upper Deschutes Basin, it appears that the faults affect ground water primarily by their influence on deposition. The resulting juxtaposition of geologic units has produced permeability contrasts across the faults. Like the stratigraphic units within the study area, the tectonic structures are the result of complex volcano-tectonic interactions over a relatively long (30 million years) time period. However, most of the faults seen at the surface have formed as a result of tectonic activity within the past 7.5 million years. The ages of deformation within the study area generally are not precisely known but are constrained by radiometric ages on many stratigraphic units. The major tectonic structures within the study area include the Cyrus Springs fault zone, Brothers fault zone, faults forming the west side of Green Ridge, the Sisters fault zone (including the Tumalo fault), faults forming the western side of Walker Rim, the Chemult graben, and the La Pine and Shukash grabens (fig. 8). These are all described in the following paragraphs.

\section{Cyrus Springs Fault Zone}

The Cyrus Springs fault zone is by far the oldest structural feature identified within the study area. The fault zone cuts the John Day Formation and is believed to have become active between 28 and 30 million years ago (Smith and others, 1998). Faults within the Cyrus Springs fault zone are oriented primarily N $45^{\circ}$ to $60^{\circ} \mathrm{E}$, and are exposed along a trace only about 3.5 miles long. However, the fault zone exhibits about $3,800 \mathrm{ft}$ of vertical offset and possibly over 4 miles of right-lateral displacement (Smith and others, 1998). Movement on the fault zone appears to be restricted to mid-Oligocene to mid-Miocene time because overlying Deschutes Formation deposits at both ends of the fault trace are undeformed (Sherrod and others, in press). Thus, the Cyrus Springs fault zone is contained entirely within low-permeability strata and does not appear to have any direct influence on the regional ground-water flow system.

\section{Brothers Fault Zone}

The Brothers fault zone is a northwest-trending zone of closely spaced en echelon normal faults that extend from the southeastern part of Oregon to the Deschutes Basin, a distance of about 150 miles (Walker and Nolf, 1981). The northern terminus of the fault zone is within the study area and is shown on figure 8 . The Brothers fault zone has been recognized as a significant tectonic feature in Oregon (Walker, 1969; Lawrence, 1976; Walker and Nolf, 1981). Walker and Nolf (1981) speculate that the normal faults and many of the volcanic vents along the fault zone are surface manifestations of deformation on a large, deeply buried structure, and that the associated volcanic vents along the fault zone indicate episodic activity over time. Individual faults within the fault zone offset strata between 3 and $300 \mathrm{ft}$ (Walker and Nolf, 1981).

The northernmost segment of the Brothers fault zone is near the boundary between late Miocene and younger rocks to the southwest, and the middle Miocene and older Blue Mountains Province to the northeast. The northwest terminus of the Brothers fault zone offsets 7.5-million-year-old lava on Horse Ridge in the southeast corner of the study area. However, the fault zone does not offset the 0.7-million-year-old lava flows at the Badlands just north of Horse Ridge.

Ground-water flow does not appear to be affected by the Brothers fault zone within the study area. Sparse ground-water-level data within the fault zone suggest that ground-water flow is not interrupted as it crosses several fault strands within the fault zone (fig. 9).

\section{Sisters Fault Zone}

The Sisters fault zone trends north-northwest from the vicinity of Newberry Volcano to Black Butte, a distance of about 37 miles (fig. 8). The fault zone comprises about 50 mapped faults and has one fault, the Tumalo fault, that is continuous for about 30 miles (Sherrod and others, in press). The youngest unit offset by the Sisters fault zone is an alluvial deposit near Upper Tumalo Reservoir (Taylor, 1981). The faulted alluvium is overlain by undeformed alluvium, and the youngest probable age of deformation is about 25,000 years (Sherrod and others, in press). 


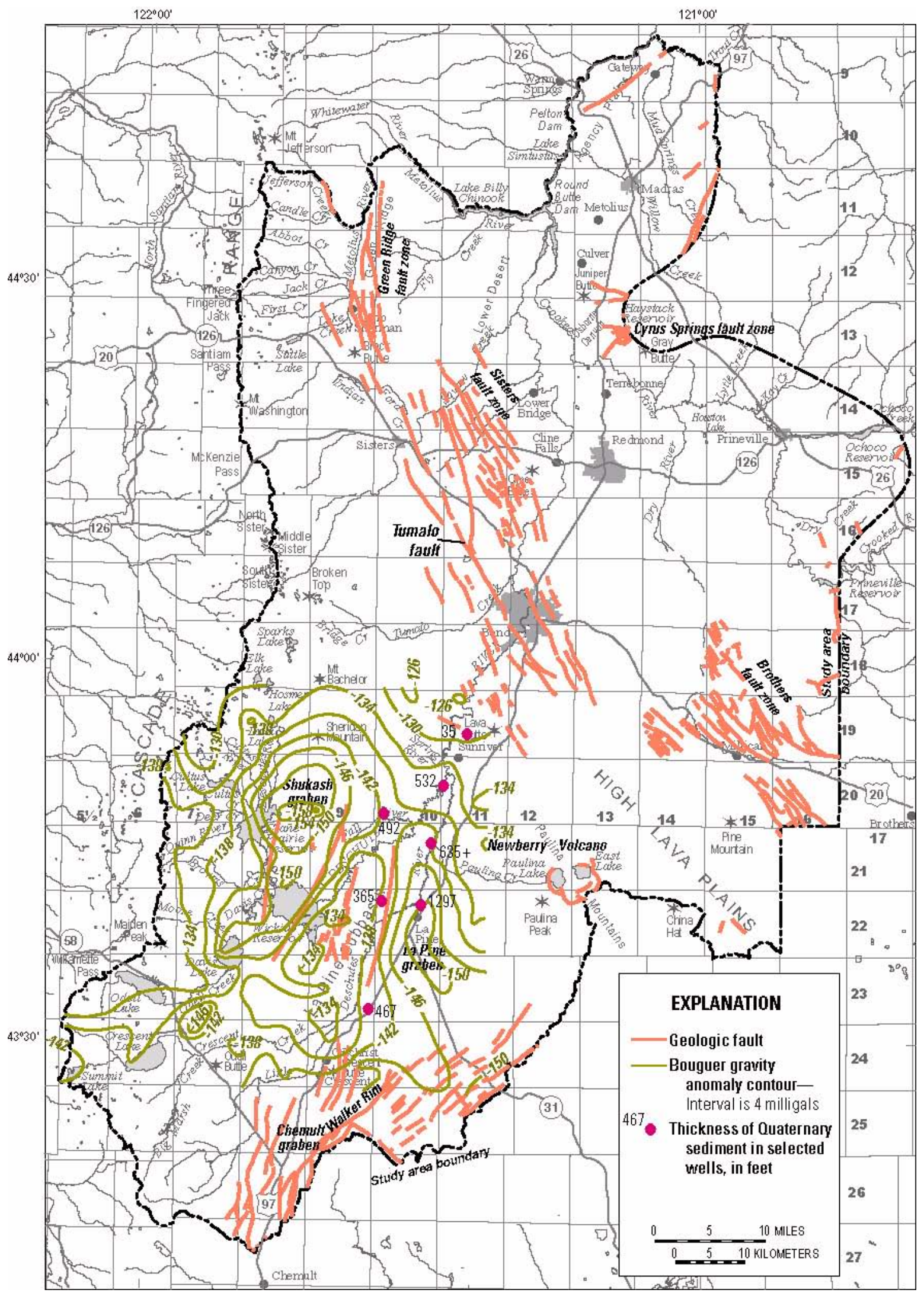

Figure 8. Major tectonic structures in the upper Deschutes Basin, Oregon. (Gravity contours from Pitts and Couch, 1978) 


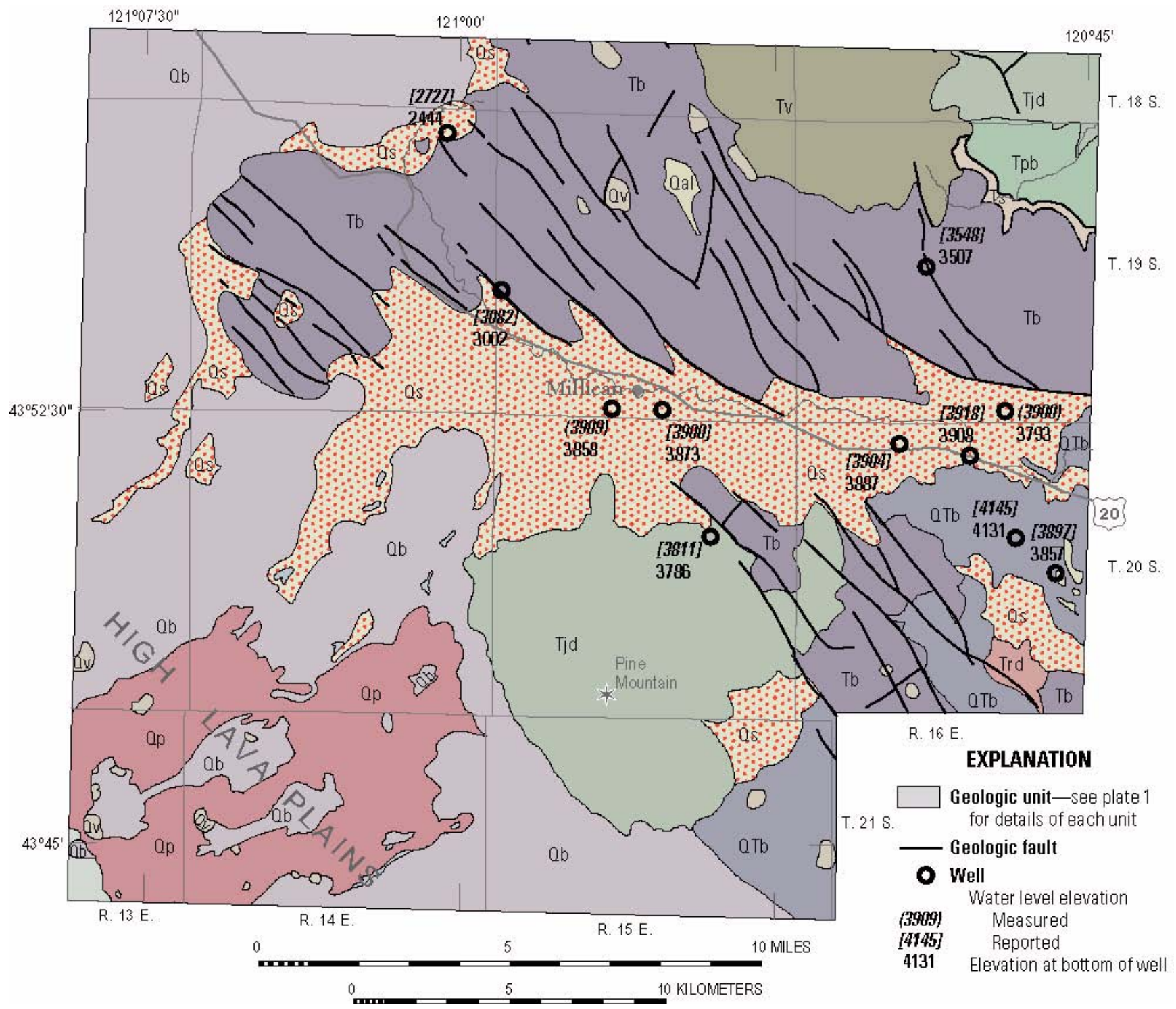

Figure 9. Geologic units, fault traces, and hydraulic head in wells in the vicinity of the Brothers fault zone, upper Deschutes Basin, Oregon.

Movement along the Tumalo fault was chiefly dip slip. As much as 180 to $200 \mathrm{ft}$ of dip separation has occurred near upper Tumalo Reservoir. Quaternary lava flows younger than 0.78 million years, exposed in the same area, exhibit escarpments of 20 to $30 \mathrm{ft}$ (Sherrod and others, in press).

It is difficult to know what, if any, direct influence the Sisters fault zone has on regional groundwater flow. The trace of the fault zone is coincident with the eastern edge of a steep precipitation (and recharge) gradient. The net result is that the water-table gradient also changes slope in the vicinity of the Sisters fault zone, obscuring any direct effects from the fault zone itself (fig. 10).

The westernmost fault strands of the Sisters fault zone that exhibit down-to-the-west displacement (notably the Tumalo fault) were partial barriers to streams flowing eastward from the Cascade Range and, thus, formed depositional centers for Pleistocene volcanic and glacial outwash deposits. The permeability contrast between the Pleistocene deposits and the upper Miocene to Pliocene Deschutes Formation strata has formed several shallow aquifers, the most extensive of which is in the glacial outwash in the vicinity of Sisters (fig. 11). 


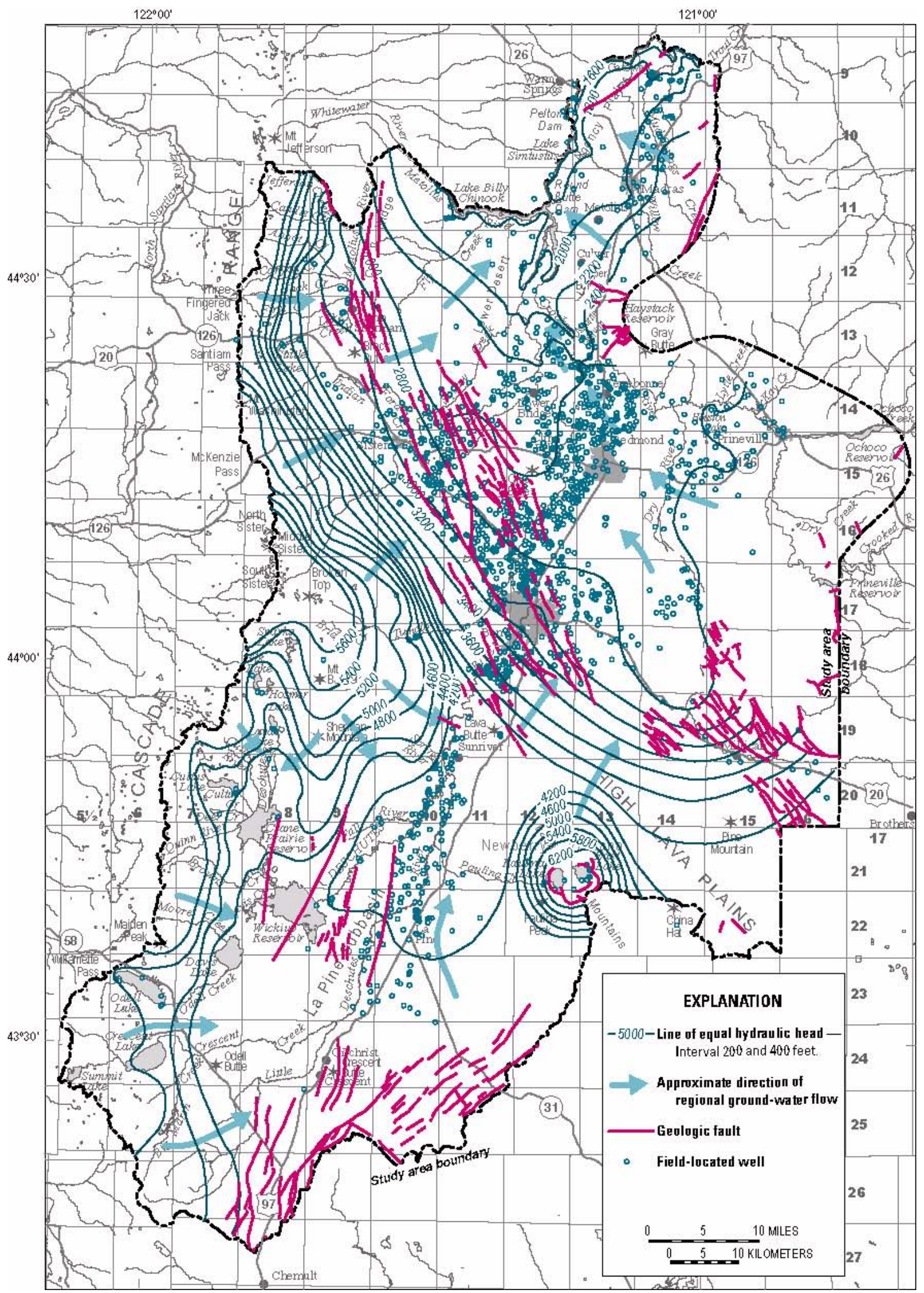

Figure 10. Generalized lines of equal hydraulic head, ground-water flow directions, and major tectonic structures in the upper Deschutes Basin, Oregon. (Head contours from Gannett and others, 2001) 

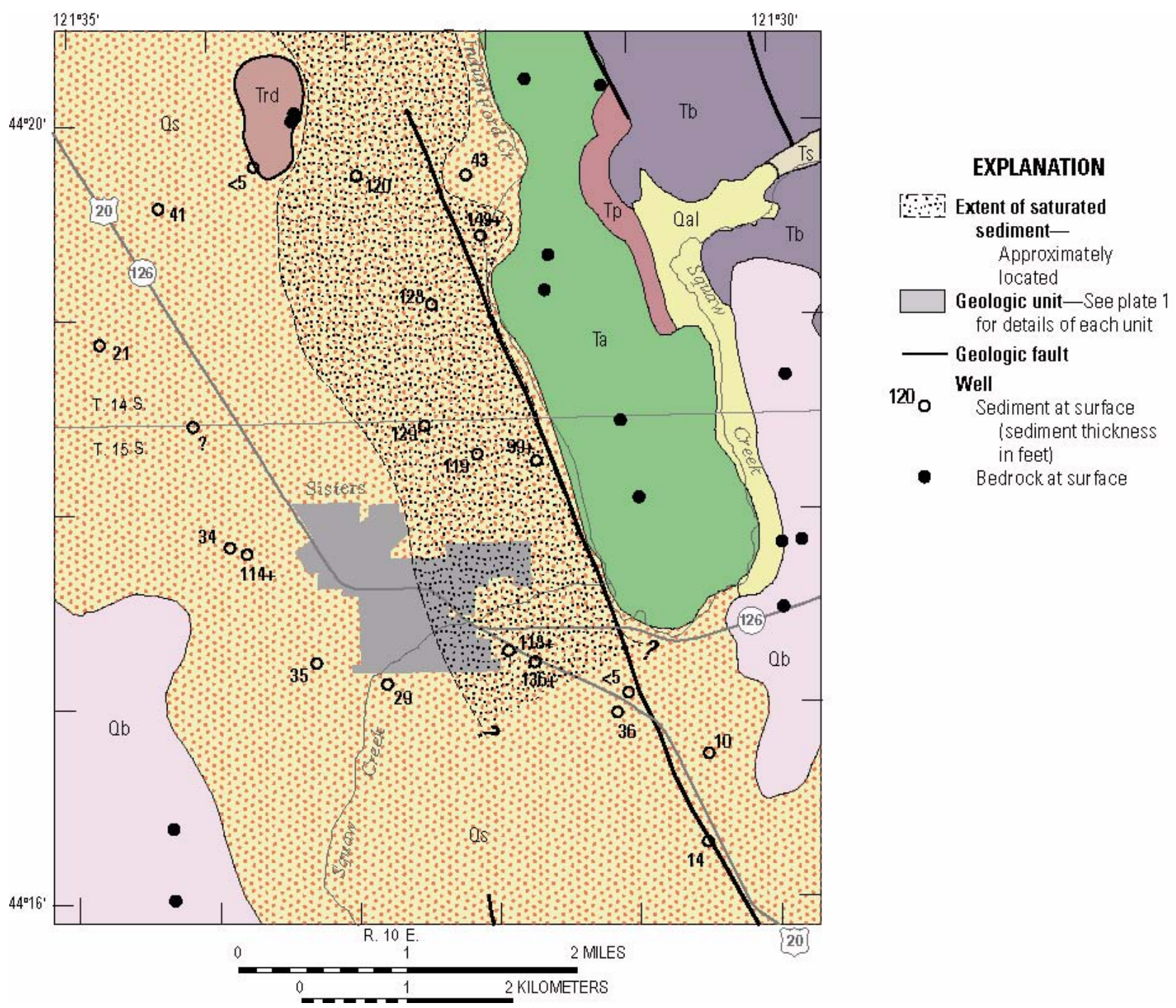

Figure 11. Thickness of Quaternary sediment in field-located wells and the approximate extent of saturated sediment in the Sisters area, upper Deschutes Basin, Oregon.

\section{Green Ridge}

Green Ridge is a 20-mile-long, north-trending escarpment in the northwestern part of the study area (fig. 8). The feature represents the east margin of an intra-arc graben within the Cascade Range (Allen, 1966; Taylor, 1981; Smith and others, 1987). Initial subsidence of the High Cascade Range occurred about 5.4 million years ago (Smith and others, 1987). According to Smith and others (1987), Green Ridge and its east-sloping flank are all that remains in this area of a once-stable Pliocene-age eastern Cascade Range foothills. The region to the west of Green Ridge has dropped as much as 3,000 ft (Conrey, 1985).

Virtually all the ground water that is recharged in the Metolius subbasin discharges west of the Green
Ridge escarpment (Gannett and others, 2001). Over $1,000 \mathrm{ft}^{3} / \mathrm{s}$ of ground water discharges to the Metolius River and its tributary springs and creeks between the headwaters and the confluence with Jefferson Creek, 10 miles downstream. The precise mechanism that causes the discharge is not clearly known, but it probably is related to the Green Ridge structure. One possible explanation is that a permeability contrast exists between Deschutes Formation rocks forming the escarpment and flooring the eastern margin of the intraarc graben and the Pliocene and Pleistocene volcanic and volcaniclastic material filling the graben. Another explanation is that the fault zone and possibly related dikes form a boundary to horizontal ground-water flow. The most likely explanation is that a combination of mechanisms is responsible. 


\section{Walker Rim Fault Zone and Chemult Graben}

The Walker Rim fault zone is a north- to northeast-trending series of basin and range normal faults south of La Pine (fig. 8). The faults form an arcuate pattern with generally down-to-the-west displacement (MacLeod and Sherrod, 1992). The surface traces of the faults terminate on the southern flank of Newberry Volcano. The Walker Rim fault zone forms the east boundary of the Chemult graben. The Chemult graben is about 6 miles wide; its floor is composed of a series of bedrock horst blocks that are thickly mantled by Holocene pyroclastic flow and fall deposits from Mount Mazama (MacLeod and Sherrod, 1992).

Water-level data indicate that a low gradient ground-water divide may be coincident with the Walker Rim fault zone. However, only sparse groundwater elevation data exist in and around the Walker Rim fault zone, so precise characteristics of the ground-water flow system in the vicinity of the Walker Rim fault zone are uncertain.

\section{La Pine and Shukash Grabens}

The La Pine and Shukash grabens are two sideby-side, north-northeast trending, elongate structures separated by a north-northeast trending horst block (MacLeod and Sherrod, 1992). The grabens are located in the southwestern part of the study area generally referred to as the La Pine subbasin (fig. 8). Most of what is known about the subsurface in the grabens is based on geophysical data (Couch and Foote, 1985). According to Couch and Foote (1985), the grabens possess 1,800 to $2,400 \mathrm{ft}$ of structural relief that has been filled with sediment. That conclusion is supported in the La Pine area by data from deep (555 to 1,460 ft) water wells. MacLeod and Sherrod (1992) speculate that the La Pine graben marks the structural boundary between the Basin and Range and Cascade Range Provinces.

The La Pine and Shukash grabens formed a depositional center filled by low-permeability, finegrained sediment. These deposits have resulted in a permeability contrast within the La Pine subbasin that affects ground-water flow. Hundreds of feet of lowpermeability, fine-grained sediment in the grabens is juxtaposed and interbedded with permeable lava from Newberry Volcano and the Cascade Range at the graben margins. Many spring complexes occur along the western margin of the Shukash graben. It is likely that these springs occur where water moving eastward from the Cascade Range encounters the low-permeability, basin-filling deposits and is diverted to the surface. The fine-grained basin-filling deposits are overlain by a thin deposit of more permeable, coarser-grained sediment. This stratigraphic sequence results in a shallow, flat water table within the La Pine subbasin. At the northern end of the La Pine subbasin, near Sunriver, the water table slopes steeply towards the north and the depth to water increases (fig. 10) (also see Gannett and others, 2001).

\section{HYDROGEOLOGY}

\section{John Day Formation}

Ground-water flow in the study area is strongly influenced by a low-permeability boundary at the top of the John Day Formation (Tjd on plate 1). Regional ground water, which flows in a northerly direction across most of the study area, ultimately discharges to the surface when it encounters the low-permeability rocks of the John Day Formation. The same low permeability of the rocks also makes the unit a poor source of water.

The John Day Formation is a sequence of upper Eocene to lower Miocene volcanic, volcaniclastic, and sedimentary rocks that is locally up to $4,000 \mathrm{ft}$ thick (Robinson and others, 1984). The John Day Formation was subdivided into members by Peck (1964) and Smith and others (1998) and into facies by Robinson and others (1984) within and adjacent to the upper Deschutes Basin study area. The upper Deschutes Basin study area borders parts of the western and southern facies of Robinson and others (1984), and contain Peck's (1964) Members A through I (Smith, 1987; Smith and Hayman, 1987; Smith and others, 1998). Lapilli tuff and tuffaceous claystone make up about 70 to 75 percent of the western facies of the John Day Formation (Robinson and others, 1984). These pyroclastic deposits were once almost entirely glass-rich volcanic ash. Over time, the glass has been weathered or diagenetically altered to clay (Robinson and others, 1984), and the porosity and permeability of the formation has been greatly reduced as a result.

The John Day Formation within the northern part of the study area consists of the western facies deposits and has been correlated to Member I of Peck (1964) (Smith, 1987; Smith and Hayman, 1987). Smith (1987), and Smith and Hayman (1987) describe the unit as tuff, lapillistone, fine-grained volcanic sandstone, and mudstone. 
Almost the entire sequence of John Day Formation rocks (units $\mathrm{A}-\mathrm{H}$ ) has been recognized along the northeastern edge of the study area from the vicinity of Smith Rock north to Haystack Reservoir (Smith and others, 1998). Additionally, Powell Buttes has been correlated with the John Day Formation (Robinson and others, 1984; Sherrod and others, in press). Powell Buttes consist of a sequence of rhyolite domes and associated strata (Sherrod and others, in press) about $28 \mathrm{Ma}$ in age, within the age range of John Day Formation unit G.

The John Day Formation in the vicinity of Prineville has been subdivided only on the basis of general compositional characteristics. Swanson (1969) described the John Day rocks near Prineville as principally bedded tuff, lapilli tuff, tuffaceous sediment; welded rhyolitic ash-flow tuff; and rhyolite and dacite flows and domes.

John Day Formation rocks usually are easy to determine from well reports because of the predominance of material described by the drillers as clay or claystone. The wells in the northern part of the study area drilled into John Day Formation clay and claystone are presumably constructed into unit I, based on surface geologic mapping. The predominance of clay, in addition to the generally silicic nature of John Day Formation rocks, also produce a relatively high gamma signature on natural gamma logs. Figure 12 shows the natural gamma trace for two wells (10S/12E-01CDD2, well no. 3, plate 1; and 11S/14E-34ABC, well no.7, plate 1) in the northern part of the study area drilled into the John Day Formation.

Alteration of the rocks within the John Day Formation has affected the unit's permeability and, therefore, well yields. Published hydraulic conductivity estimates for John Day Formation rocks in the upper Deschutes Basin range from 0.01 to $0.1 \mathrm{ft} / \mathrm{d}$ (feet per day) (Bolke and Laenen, 1989). Many of the wells open to water-bearing zones within the John Day rocks have yields that are less than $10 \mathrm{gal} / \mathrm{min}$ (gallons per minute). The exceptions to this generalization occur where wells intercept water-bearing fractures within some ignimbrite units. A notable example is just outside the study area near Hay Creek, 15 miles northeast of Madras, where wells yield up to a few hundred gallons per minute (Norton, 1988). Those conditions may also exist locally within the study area as well. However, the few good aquifers within the John Day Formation are of limited extent, are poorly connected, and probably have limited long-term water-supply capacity. The overall low permeability of the John Day Formation makes it a boundary to the regional groundwater flow system in the upper Deschutes Basin.

\section{Prineville Basalt}

The Prineville Basalt (Tpb on plate 1) is the oldest stratigraphic unit that bears substantial water in the upper Deschutes Basin. The Prineville Basalt is utilized as a source of ground water in three areas: just north of Powell Buttes, between Powell Buttes and the Crooked River, and near Gateway, northeast of Madras.

The Prineville Basalt is a series of middle Miocene lava flows that have a maximum thickness of nearly $700 \mathrm{ft}$ within the upper Deschutes Basin study area (Smith, 1986b). Prineville Basalt flows cover an area of at least $4,200 \mathrm{mi}^{2}$, from just south of Bowman Dam to the Portland area (Hooper and others, 1993). The flows are compositionally similar to each other, but chemically distinct from other basalt by their exceptionally high barium and phosphorus contents (Uppuluri, 1974). Smith (1986b) identified variations in silica $\left(\mathrm{SiO}_{2}\right)$ content among the flows and characterized flows as either high ( $\sim 54$ percent) or low ( $\sim 1$ percent) in $\mathrm{SiO}_{2}$. The low- $\mathrm{SiO}_{2}$ unit near Pelton Dam has an age of 15.7 Ma (Smith, 1986b). The highand low- $\mathrm{SiO}_{2}$ flows have also been identified in the northernmost part of the upper Deschutes study area near Gateway (Smith, 1986b; Smith, 1987; and Smith and Hayman, 1987).

The natural gamma log for well 09S/14E-27BBA (well no. 2, plate 1) near Gateway shows the subtle chemical differences between the high- $\mathrm{SiO}_{2}$ and low$\mathrm{SiO}_{2}$ Prineville Basalt flows (fig. 13). The gamma trace for the lava flows reflects the differences in potassium oxide $\left(\mathrm{K}_{2} \mathrm{O}\right)$ content of the two flows. According to Smith and Hayman (1987), the high-SiO $\mathrm{Sl}_{2}$ flow contains 3.3 weight percent $\mathrm{K}_{2} \mathrm{O}$ and the low- $\mathrm{SiO}_{2}$ flow contains 2.1 weight percent $\mathrm{K}_{2} \mathrm{O}$. The elevation of the contact at $190 \mathrm{ft}$ depth in well 09S/14E-27BBA is roughly coincident with the elevation of the contact between high- $\mathrm{SiO}_{2}$ and low- $\mathrm{SiO}_{2}$ flows mapped nearby by Smith and Hayman (1987). The gamma spike at approximately $230 \mathrm{ft}$ depth represents a $4 \mathrm{ft}$ sedimentary interbed that was described by the well driller as green sand and sandstone. That interbed may be part of the Simtustus Formation of Smith (1986a). 
A.

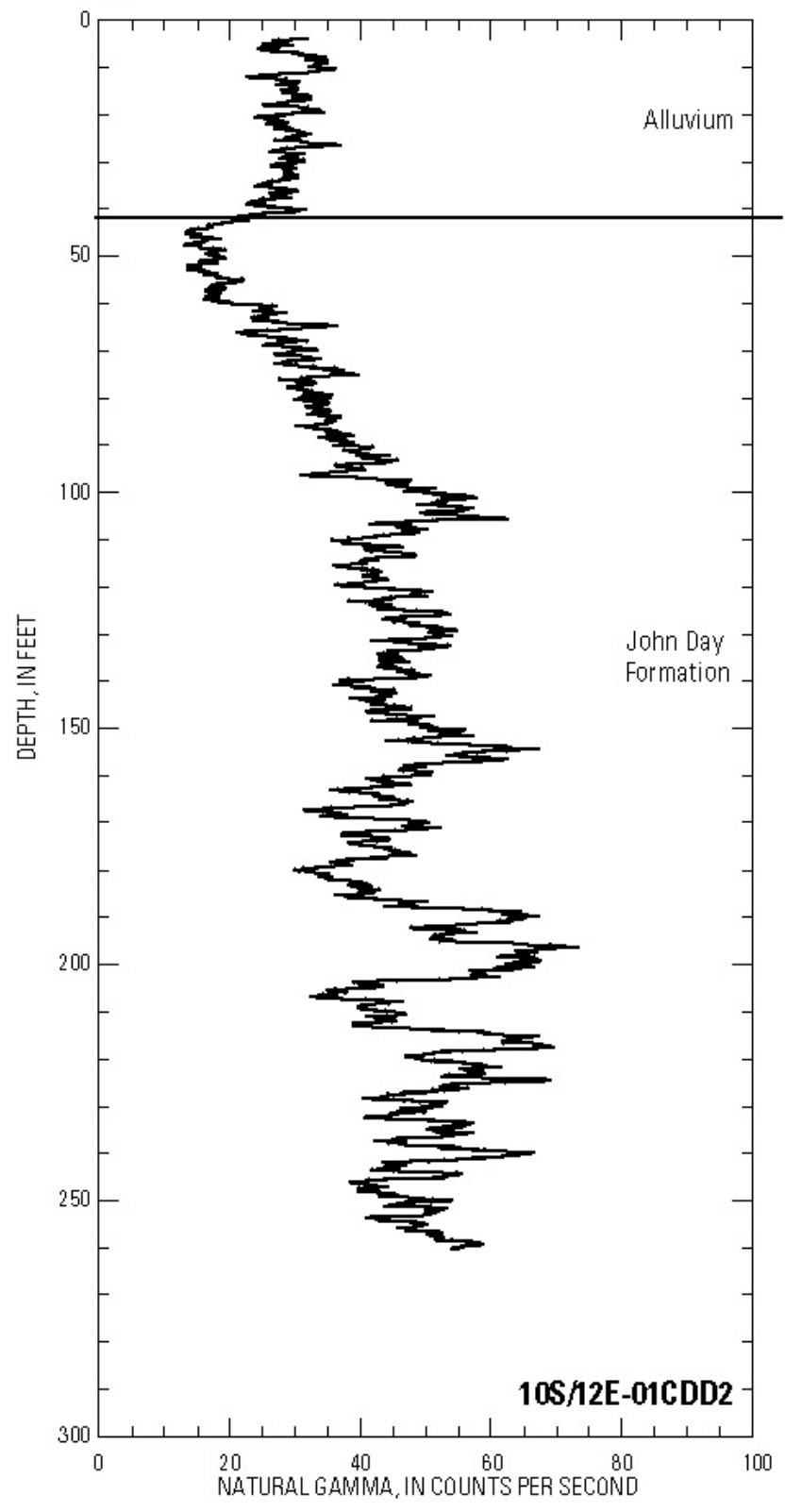

B.

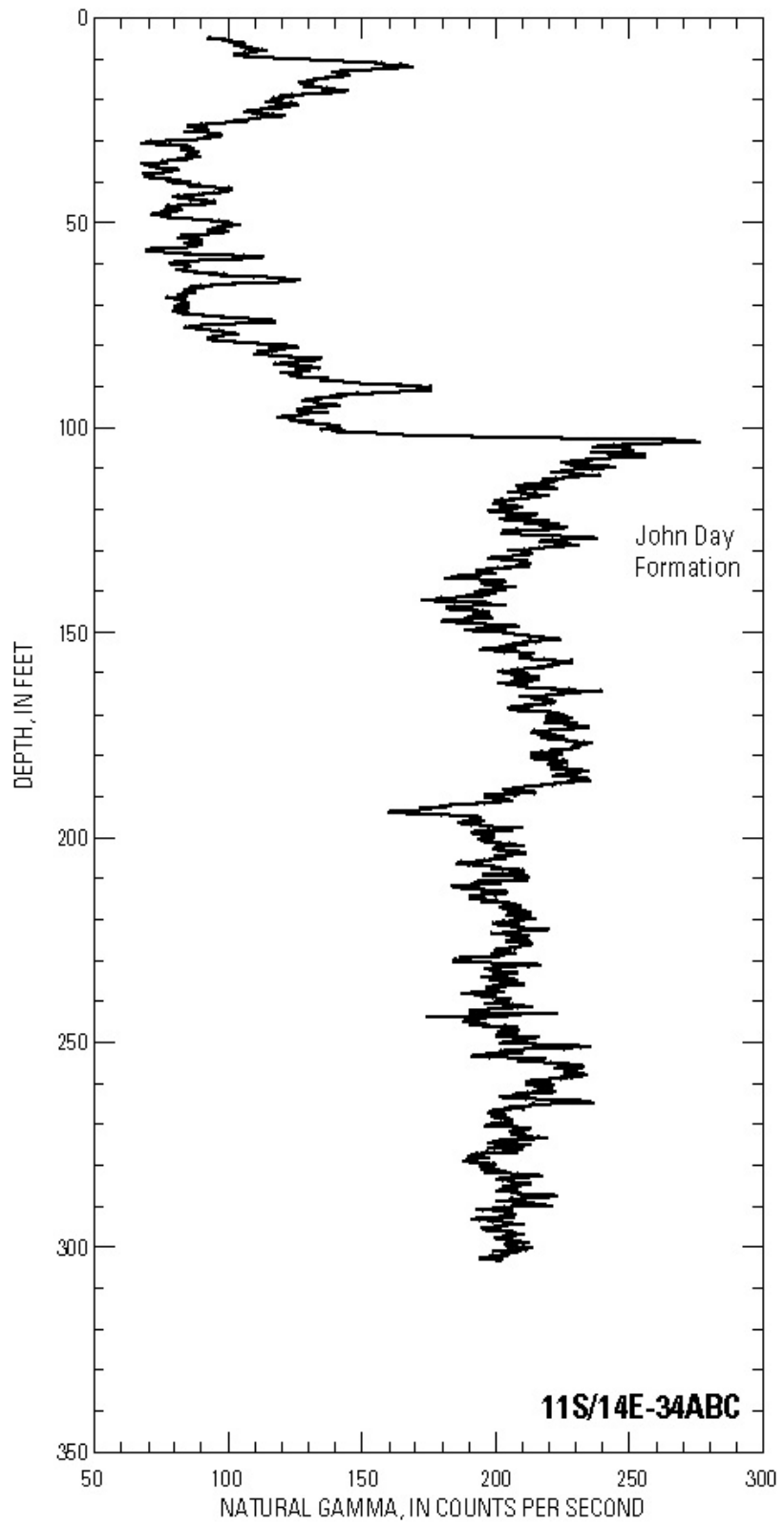

Figure 12. Natural gamma logs for wells constructed into the John Day Formation. A. Well 3 miles south of Warm Springs, Oregon (10S/12E-01CDD2, well no. 3, plate 1); B. Well 5 miles southeast of Madras, Oregon (11S/14E-34ABC, well no. 7, plate 1).

Prineville Basalt was sampled and analyzed from one well (15S/15E-23BBB, samples 14 and 15) for this study (tables 2 and 3 ). The well is located about 1 mile south of the Prineville airport (well no. 64, plate 1). The rock chemistry for the Prineville samples was typical for the low- $\mathrm{SiO}_{2}$ Bowman Dam-type lava.

Hydraulic conductivity estimates for middle Miocene basalt flows in the upper Deschutes Basin, assumed to be Prineville Basalt, range from 1 to $50 \mathrm{ft} / \mathrm{d}$ (Bolke and Laenen, 1989). Wells constructed into water-bearing zones within the Prineville Basalt typically yield 10 to $30 \mathrm{gal} / \mathrm{min}$. The amount is generally inadequate for irrigation but is satisfactory for domestic and stock uses.

\section{Deschutes Formation}

The Deschutes Formation and age-equivalent deposits are by far the most widely used ground-waterbearing units in the study area. The deposits occur at 


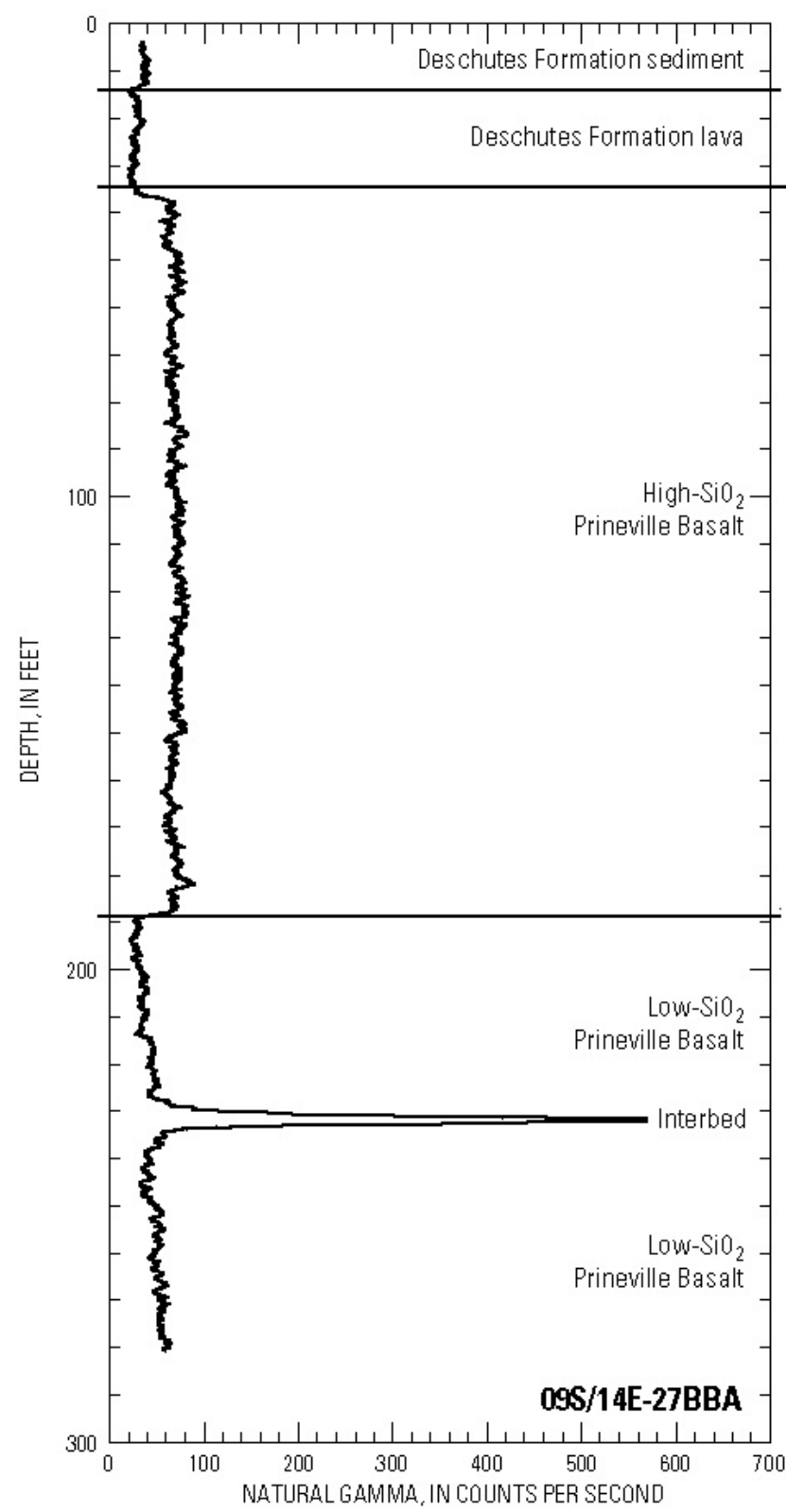

Figure 13. Natural gamma log for a well constructed into the Prineville Basalt near Gateway, Oregon (09S/14E-27BBA, well no. 2, plate 1).

the surface or at relatively shallow depth in the subsurface throughout much of the study area north and east of the Sisters fault zone (fig. 14). The Deschutes Formation consists of a variety of volcanic and sedimentary deposits ranging in age from late Miocene to Pliocene (approximately 7.5 to 4.0 million years before present). However, our map of Deschutes Formation and age-equivalent deposits (plate 1) also include some younger volcanic units. We include the basalt of Redmond and basalt of Dry River, basaltic andesite of Little Squaw Back and Squaw Back Ridge, and andesite of McKinney Butte (Sherrod and others, in press); middle Pliocene and lower Pleistocene basalt flows and basaltic vent rocks of Swanson (1969); and late Pliocene and Pleistocene basalt flows, northwest of Millican (Walker and others, 1967). Our discussion of the Deschutes Formation also includes volcanic units (primarily lava flows) that are found on the High Lava Plains but are age equivalent and compositionally similar to the Deschutes Formation rocks. In fact, some of the lava flows within the Deschutes Formation may have originated from the High Lava Plains Province (Smith, 1986b).

As mentioned in a previous section, Smith (1986b) subdivided the Deschutes Formation into three depositional environments: inactive-basin margin, arc-adjacent plain, and ancestral Deschutes River (see fig. 7). Smith also recognized a fourth subdivision of the Deschutes Formation: the proximal volcanic rocks (predominately lava flows) that form the bulk of Green Ridge. Similar layered lava flows are the predominant Deschutes Formation strata that occur at depth in much of the southeastern part of the study area south of Long Butte. A few of the volcanic eruptive centers are exposed at the surface, such as Long Butte, Awbrey Butte, Horse Ridge, and West Butte, but many more are presumably buried beneath the younger lava flows from Newberry Volcano.

Smith's subdivisions for the Deschutes Formation rocks also effectively characterize the general hydrologic properties of the unit. Generally, the permeability of Deschutes Formation rocks is lowest in the inactive-margin facies (less than $10^{2} \mathrm{ft} / \mathrm{d}$ ), and moderate to very high $\left(10^{2}\right.$ to $\left.10^{3} \mathrm{ft} / \mathrm{d}\right)$ in the arc-adjacent plain and proximal volcanic rock facies (Gannett and others, 2001). Lithologic considerations, well-yield data, and the volume of spring discharge indicate that the hydraulic conductivity of the ancestral Deschutes River facies is at least as large as the arc-adjacent plain and proximal volcanic rock facies.

\section{Inactive-Margin Facies}

The low permeability of the inactive-margin material is a result of the fine-grained character of the clastic and pyroclastic deposits derived principally from the John Day Formation or as distal fallout from Cascade Range eruptions (Smith, 1986b). Most of the sediment was deposited on low-gradient alluvial fans radiating outward from uplands composed of John Day Formation. 
Table 2. Major oxide analyses of cuttings samples from selected wells in the upper Deschutes Basin, Oregon

Well location: Refer to "Well- and "Spring-Location System" for explanation. Sample depth: Given in feet below land surface. Stratigraphic Unit: DF, Deschutes Formation; CR, Cascade Range; NV, Newberry Volcano.

\begin{tabular}{|c|c|c|c|c|c|c|c|c|c|c|c|c|c|c|c|c|}
\hline \multirow{2}{*}{$\begin{array}{l}\text { Sample } \\
\text { number }\end{array}$} & \multirow{2}{*}{$\begin{array}{c}\text { Well } \\
\text { number }\end{array}$} & \multirow[b]{2}{*}{ Well location } & \multirow{2}{*}{$\begin{array}{c}\text { Sample } \\
\text { depth }\end{array}$} & \multirow[b]{2}{*}{ Sample name } & \multirow[b]{2}{*}{ Stratigraphic unit } & \multicolumn{10}{|c|}{ Major oxides (in weight percent) } & \multirow[b]{2}{*}{ Total } \\
\hline & & & & & & $\mathrm{SiO}_{2}$ & $\mathrm{Al}_{2} \mathrm{O}_{3}$ & $\mathrm{TiO}_{2}$ & $\mathrm{FeO}$ & MnO & $\mathrm{CaO}$ & MgO & $\mathrm{K}_{2} \mathrm{O}$ & $\mathrm{Na}_{2} \mathrm{O}$ & $\mathrm{P}_{2} \mathrm{O}_{5}$ & \\
\hline 1 & 9 & $12 \mathrm{~S} / 12 \mathrm{E}-33 \mathrm{ACB}$ & 250 & RMCOS1250 & DF lava & 49.09 & 17.03 & 1.044 & 9.19 & 0.173 & 11.83 & 8.97 & 0.33 & 2.25 & 0.142 & 100.05 \\
\hline 2 & 9 & $12 \mathrm{~S} / 12 \mathrm{E}-33 \mathrm{ACB}$ & 280 & RMCOS 1280 & DF lava & 49.27 & 17.00 & 1.269 & 9.77 & .170 & 10.64 & 8.41 & .31 & 2.62 & .213 & 99.67 \\
\hline 3 & 9 & $12 \mathrm{~S} / 12 \mathrm{E}-33 \mathrm{ACB}$ & 415 & RMCOS1415 & DF-Pelton basalt & 48.54 & 16.27 & 1.812 & 11.68 & .195 & 9.99 & 7.19 & .52 & 3.07 & .528 & 99.80 \\
\hline 4 & 9 & $12 \mathrm{~S} / 12 \mathrm{E}-33 \mathrm{ACB}$ & 585 & LITOPAL585 & DF- Pelton basalt & 48.18 & 15.91 & 1.621 & 11.46 & .195 & 9.74 & 7.58 & .49 & 2.95 & .404 & 98.53 \\
\hline 5 & 9 & $12 \mathrm{~S} / 12 \mathrm{E}-33 \mathrm{ACB}$ & 725 & LITOPAL725 & DF-Pelton basalt & 48.60 & 16.07 & 1.499 & 10.79 & .190 & 10.21 & 8.01 & .47 & 2.78 & .312 & 98.93 \\
\hline 6 & 14 & $13 \mathrm{~S} / 12 \mathrm{E}-36 \mathrm{BDB}$ & $448-460$ & LITEA448460 & DF-Opal Springs basalt & 50.20 & 17.58 & .972 & 8.30 & .171 & 11.46 & 8.20 & .39 & 2.59 & .129 & 99.99 \\
\hline 7 & 54 & $15 \mathrm{~S} / 13 \mathrm{E}-20 \mathrm{AAD}$ & 520 & LITRED520 & DF lava & 50.04 & 17.28 & 1.146 & 8.75 & .167 & 10.69 & 7.49 & .46 & 3.09 & .226 & 99.34 \\
\hline 8 & 54 & 15S/13E-20AAD & 670 & LITRED670 & DF lava & 49.98 & 16.54 & 1.573 & 10.36 & .181 & 9.68 & 6.41 & .71 & 3.29 & .394 & 99.12 \\
\hline 9 & 54 & 15S/13E-20AAD & $775-780$ & LITRE775780 & DF lava & 48.12 & 15.88 & 1.222 & 10.09 & .182 & 10.91 & 9.96 & .26 & 2.51 & .175 & 99.31 \\
\hline 10 & 60 & 15S/14E-03BAA & $294-320$ & LITIS294320 & DF lava & 48.55 & 17.01 & .960 & 9.09 & .170 & 11.22 & 10.32 & .16 & 2.42 & .127 & 100.03 \\
\hline 11 & 61 & $15 \mathrm{~S} / 14 \mathrm{E}-14 \mathrm{CDC}$ & 279 & LITSM279 & DF lava & 48.66 & 17.33 & .989 & 9.00 & .174 & 11.44 & 9.07 & .22 & 2.59 & .142 & 99.62 \\
\hline 12 & 61 & 15S/14E-14CDC & $377-400$ & LITSM377400 & DF lava & 48.98 & 17.11 & 1.240 & 9.39 & .176 & 10.68 & 8.15 & .39 & 2.84 & .263 & 99.21 \\
\hline 13 & 64 & $15 \mathrm{~S} / 15 \mathrm{E}-23 \mathrm{BBB}$ & $240-250$ & RCPRTB240250 & DF lava & 48.17 & 15.82 & 1.994 & 12.11 & .209 & 9.26 & 8.09 & .55 & 3.17 & .469 & 99.84 \\
\hline 14 & 64 & $15 \mathrm{~S} / 15 \mathrm{E}-23 \mathrm{BBB}$ & $270-280$ & RCPRTB 270280 & Prineville Basalt & 51.05 & 13.81 & 2.681 & 12.13 & .229 & 7.97 & 4.19 & 1.79 & 3.32 & 1.447 & 98.62 \\
\hline 15 & 64 & $15 \mathrm{~S} / 15 \mathrm{E}-23 \mathrm{BBB}$ & $370-380$ & RCPRTB370380 & Prineville Basalt & 51.32 & 13.84 & 2.710 & 11.89 & .230 & 8.04 & 3.98 & 1.85 & 3.36 & 1.468 & 98.69 \\
\hline 16 & 66 & $16 \mathrm{~S} / 10 \mathrm{E}-13 \mathrm{BBB} 2$ & $218-254$ & RCNMN218254 & CR lava & 53.36 & 17.17 & 1.639 & 9.11 & .156 & 8.39 & 4.87 & 1.04 & 3.99 & .311 & 100.04 \\
\hline 17 & 70 & $16 \mathrm{~S} / 11 \mathrm{E}-18 \mathrm{BAC}$ & $347-360$ & RCMCT347360 & DF lava & 54.55 & 17.02 & 1.480 & 9.13 & .154 & 7.99 & 3.87 & 1.05 & 4.29 & .375 & 99.91 \\
\hline 18 & 71 & 16S/11E-33DDD & $195-203$ & RCPT-195203 & DF lava & 54.81 & 16.06 & 1.965 & 10.47 & .173 & 7.25 & 3.25 & 1.14 & 4.22 & .416 & 99.75 \\
\hline 19 & 71 & 16S/11E-33DDD & 468 & RC-PT-468 & DF lava & 52.24 & 15.34 & 2.310 & 11.92 & .219 & 8.16 & 4.06 & .73 & 4.13 & .519 & 99.63 \\
\hline 20 & 83 & $16 \mathrm{~S} / 14 \mathrm{E}-17 \mathrm{CDD}$ & 332 & PB332 & DF lava & 48.95 & 17.39 & .714 & 8.15 & .161 & 12.63 & 9.95 & .15 & 2.08 & .073 & 100.25 \\
\hline 21 & 83 & 16S/14E-17CDD & 411 & PB411 & DF lava & 49.42 & 16.89 & 1.487 & 10.46 & .188 & 9.99 & 7.31 & .57 & 3.08 & .332 & 99.72 \\
\hline 22 & 84 & $16 \mathrm{~S} / 14 \mathrm{E}-21 \mathrm{BBD}$ & $437-478$ & LITMR437478 & DF lava & 48.27 & 17.49 & .982 & 9.14 & .178 & 11.53 & 9.14 & .24 & 2.50 & .178 & 99.64 \\
\hline 23 & 86 & $17 \mathrm{~S} / 11 \mathrm{E}-24 \mathrm{DCA}$ & 200 & RCAWG200 & DF lava & 54.24 & 18.17 & 1.315 & 8.46 & .155 & 8.68 & 4.66 & .90 & 3.59 & .358 & 100.53 \\
\hline 24 & 86 & 17S/11E-24DCA & 320 & RCAWG320 & DF lava & 51.95 & 17.74 & 1.430 & 9.98 & .166 & 8.61 & 5.20 & .87 & 3.40 & .397 & 99.74 \\
\hline 25 & 86 & 17S/11E-24DCA & $400-410$ & RCAWG400410 & DF lava & 52.12 & 18.46 & 1.425 & 9.32 & .153 & 8.76 & 4.32 & .90 & 3.67 & .600 & 99.73 \\
\hline 26 & 86 & 17S/11E-24DCA & $510-520$ & RCAWG510520 & DF lava & 52.59 & 18.17 & 1.380 & 9.21 & .156 & 8.67 & 4.40 & 1.03 & 3.70 & .606 & 99.91 \\
\hline 27 & 86 & 17S/11E-24DCA & $820-830$ & RCAWG820830 & DF lava & 53.29 & 18.20 & 1.295 & 8.83 & .157 & 9.08 & 4.53 & 1.07 & 3.57 & .526 & 100.55 \\
\hline 28 & 89 & 17S/12E-11DDB & $143-167$ & RCNLN143167 & NV lava & 50.35 & 16.38 & 1.335 & 9.09 & .163 & 9.46 & 8.87 & .51 & 3.27 & .259 & 99.69 \\
\hline 29 & 89 & 17S/12E-11DDB & $233-348$ & RCNLN233348 & DF lava & 49.92 & 17.07 & 1.641 & 10.48 & .180 & 10.13 & 7.23 & .34 & 3.46 & .231 & 100.68 \\
\hline 30 & 89 & 17S/12E-11DDB & $348-412$ & RCNLN348412 & DF lava & 49.51 & 16.59 & 1.479 & 9.81 & .166 & 9.67 & 8.98 & .44 & 3.35 & .312 & 100.31 \\
\hline
\end{tabular}


Table 2. Major oxide analyses of cuttings samples from selected wells in the upper Deschutes Basin, Oregon-Continued

Well location: Refer to "Well- and "Spring-Location System" for explanation. Sample depth: Given in feet below land surface. Stratigraphic Unit: DF, Deschutes Formation; CR, Cascade Range; NV, Newberry Volcano.

\begin{tabular}{|c|c|c|c|c|c|c|c|c|c|c|c|c|c|c|c|c|}
\hline \multirow{2}{*}{$\begin{array}{l}\text { Sample } \\
\text { number }\end{array}$} & \multirow{2}{*}{$\begin{array}{c}\text { Well } \\
\text { number }\end{array}$} & \multirow[b]{2}{*}{ Well location } & \multirow{2}{*}{$\begin{array}{c}\text { Sample } \\
\text { depth }\end{array}$} & \multirow[b]{2}{*}{ Sample name } & \multirow[b]{2}{*}{ Stratigraphic unit } & \multicolumn{10}{|c|}{ Major oxides (in weight percent) } & \multirow[b]{2}{*}{ Total } \\
\hline & & & & & & $\mathrm{SiO}_{2}$ & $\mathrm{Al}_{2} \mathrm{O}_{3}$ & $\mathrm{TiO}_{2}$ & $\mathrm{FeO}$ & MnO & $\mathrm{CaO}$ & $\mathrm{MgO}$ & $\mathrm{K}_{2} \mathrm{O}$ & $\mathrm{Na}_{2} \mathrm{O}$ & $\mathbf{P}_{2} \mathrm{O}_{5}$ & \\
\hline 31 & 89 & 17S/12E-11DDB & $465-505$ & RCNLN465505 & DF lava & 50.28 & 16.89 & 1.439 & 9.65 & .164 & 9.51 & 8.22 & .55 & 3.33 & .305 & 100.34 \\
\hline 32 & 89 & 17S/12E-11DDB & $517-520$ & RCNLN517520 & DF lava & 53.60 & 17.66 & 1.276 & 8.87 & .146 & 8.72 & 4.69 & .85 & 4.09 & .285 & 100.19 \\
\hline 33 & 91 & 17S/13E-20DAD & $96-125$ & RCBART96125 & NV lava & 51.16 & 16.37 & 1.397 & 9.31 & .162 & 9.35 & 8.62 & .55 & 3.25 & .290 & 100.46 \\
\hline 34 & 91 & 17S/13E-20DAD & $365-405$ & RCBAR365405 & DF lava & 50.33 & 16.82 & 1.396 & 9.71 & .166 & 9.45 & 7.66 & .55 & 3.29 & .307 & 99.68 \\
\hline 35 & 91 & 17S/13E-20DAD & $430-450$ & RCBAR430450 & DF lava & 52.97 & 15.13 & 2.307 & 10.81 & 215 & 8.15 & 4.46 & .75 & 4.37 & .580 & 99.74 \\
\hline 36 & 91 & 17S/13E-20DAD & $510-555$ & RCBAR510555 & DF lava & 50.12 & 16.67 & 1.475 & 9.78 & .172 & 10.13 & 7.46 & .58 & 3.01 & .357 & 99.75 \\
\hline 37 & 91 & 17S/13E-20DAD & $570-592$ & RCBAR570592 & DF lava & 49.57 & 16.85 & 1.514 & 9.52 & 175 & 10.03 & 7.75 & .55 & 3.08 & .354 & 99.39 \\
\hline 38 & 91 & 17S/13E-20DAD & $592-615$ & RCBAR592615 & DF lava & 48.77 & 16.80 & 1.559 & 10.85 & .178 & 10.01 & 7.54 & .36 & 3.07 & .351 & 99.49 \\
\hline 39 & 92 & 17S/14E-02CAA & 125-192 & RCWOG125192 & DF lava & 48.05 & 17.32 & .698 & 8.28 & 159 & 12.37 & 10.19 & .14 & 2.13 & .066 & 99.40 \\
\hline 40 & 92 & 17S/14E-02CAA & $244-327$ & RCWOG244327 & DF lava & 48.68 & 16.31 & 1.844 & 11.62 & 197 & 10.17 & 6.95 & .46 & 3.14 & .441 & 99.81 \\
\hline 41 & 92 & 17S/14E-02CAA & $327-465$ & LITWO327465 & DF lava & 50.38 & 16.64 & 1.465 & 10.61 & 180 & 9.00 & 6.35 & .79 & 3.33 & .377 & 99.12 \\
\hline 42 & 93 & 17S/14E-11DCB & $342-530$ & LITRD342530 & DF lava & 48.74 & 16.7 & 1.715 & 10.79 & .195 & 10.13 & 7.44 & .48 & 3.11 & .412 & 99.71 \\
\hline 43 & 93 & 17S/14E-11DCB & $552-598$ & LITRD552598 & DF lava & 48.33 & 17.41 & .910 & 8.61 & 176 & 11.92 & 9.52 & .17 & 2.41 & .082 & 99.53 \\
\hline 44 & 93 & 17S/14E-11DCB & $655-675$ & LITRD655675 & DF lava & 49.45 & 17.54 & .835 & 8.31 & .161 & 11.51 & 9.29 & .25 & 2.36 & .157 & 99.86 \\
\hline 45 & 101 & 18S/12E-07DBD1 & 150 & RCCTB150 & CR lava & 53.47 & 15.81 & 1.807 & 10.29 & .172 & 7.57 & 5.13 & 1.34 & 3.74 & .411 & 99.74 \\
\hline 46 & 101 & 18S/12E-07DBD1 & 270 & RCCTB270 & CR lava & 54.23 & 17.17 & 1.499 & 9.13 & .155 & 8.33 & 4.52 & .74 & 4.38 & 247 & 100.40 \\
\hline 47 & 101 & 18S/12E-07DBD1 & 310 & RCCTB310 & $\mathrm{CR}$ lava & 54.57 & 17.08 & 1.519 & 9.12 & .156 & 8.28 & 4.4 & .74 & 4.3 & 249 & 100.41 \\
\hline 48 & 101 & 18S/12E-07DBD1 & 370 & RCCTB370 & $\mathrm{CR}$ lava & 53.89 & 16.98 & 1.617 & 9.03 & .155 & 8.51 & 4.89 & .84 & 4.06 & .302 & 100.27 \\
\hline 49 & 108 & 18S/13E-24DCB & 170-194 & RCDB-170194 & NV lava & 50.71 & 16.85 & .952 & 8.64 & 167 & 10.34 & 9.77 & .40 & 2.77 & .155 & 100.75 \\
\hline 50 & 108 & 18S/13E-24DCB & 194-226 & RCDB-194226 & NV lava & 50.37 & 16.75 & .940 & 8.98 & .169 & 10.02 & 10.08 & .42 & 2.70 & .154 & 100.58 \\
\hline 51 & 108 & 18S/13E-24DCB & $226-275$ & RCDB-226275 & NV lava & 49.37 & 16.53 & 1.000 & 9.47 & .173 & 10.03 & 9.91 & .41 & 2.73 & .161 & 99.77 \\
\hline 52 & 108 & 18S/13E-24DCB & $294-352$ & RCDB294-352 & NV lava & 49.69 & 16.78 & 1.634 & 11.45 & .190 & 8.97 & 6.73 & .56 & 3.54 & .284 & 99.83 \\
\hline 53 & 108 & 18S/13E-24DCB & $352-405$ & RCDB352-405 & NV lava & 49.41 & 16.91 & 1.62 & 10.14 & 192 & 10.10 & 7.67 & .31 & 3.28 & .267 & 99.90 \\
\hline 54 & 108 & 18S/13E-24DCB & $424-521$ & RCDB424-451 & NV lava & 50.82 & 17.23 & 1.367 & 9.22 & .162 & 9.50 & 7.59 & .57 & 3.39 & .318 & 100.17 \\
\hline 55 & 108 & 18S/13E-24DCB & $536-632$ & RCDB536-632 & NV lava & 51.19 & 17.85 & 1.017 & 8.23 & .151 & 10.08 & 7.56 & .53 & 3.08 & .186 & 99.87 \\
\hline 56 & 108 & 18S/13E-24DCB & $710-721$ & RCDB710-721 & DF-equivalent lava & 54.87 & 17.27 & 1.138 & 7.92 & .141 & 8.78 & 4.75 & 1.10 & 3.39 & .272 & 99.63 \\
\hline 57 & 108 & 18S/13E-24DCB & $721-770$ & RCDB721-770 & DF-equivalent lava & 55.04 & 17.39 & 1.095 & 7.92 & .139 & 9.14 & 5.03 & 1.08 & 3.23 & .260 & 100.32 \\
\hline 58 & 108 & 18S/13E-24DCB & $770-785$ & RCDB770-785 & DF-equivalent lava & 54.55 & 17.29 & 1.055 & 7.86 & 140 & 9.08 & 5.44 & 1.03 & 3.28 & 240 & 99.97 \\
\hline 59 & 108 & 18S/13E-24DCB & 785-904 & RCDB785-904 & DF-equivalent lava & 55.19 & 17.28 & 1.047 & 7.67 & . 142 & 9.16 & 5.78 & 1.02 & 3.31 & .243 & 100.84 \\
\hline
\end{tabular}


Table 3. Trace element analyses of cuttings samples from selected wells in the upper Deschutes Basin, Oregon

Well location: Refer to "Well- and "Spring-Location System" for explanation. Sample depth: Given in feet below land surface. Stratigraphic Unit: DF, Deschutes Formation; CR, Cascade Range;

NV, Newberry Volcano.

\begin{tabular}{|c|c|c|c|c|c|c|c|c|c|c|c|c|c|c|c|c|c|c|c|c|c|c|}
\hline \multirow{2}{*}{$\begin{array}{l}\text { Sample } \\
\text { number }\end{array}$} & \multirow{2}{*}{$\begin{array}{c}\text { Well } \\
\text { number }\end{array}$} & \multirow[b]{2}{*}{ Well location } & \multirow{2}{*}{$\begin{array}{c}\text { Sample } \\
\text { depth }\end{array}$} & \multirow[b]{2}{*}{ Sample Name } & \multirow[b]{2}{*}{ Stratigraphic Unit } & \multicolumn{17}{|c|}{ Trace elements (in parts per million) } \\
\hline & & & & & & $\mathbf{N i}$ & $\mathrm{Cr}$ & Sc & $\mathbf{V}$ & Ba & $\mathbf{R b}$ & Sr & $\mathrm{Zr}$ & $\mathbf{Y}$ & $\mathbf{N b}$ & Ga & Cu & $Z \mathbf{n}$ & $\mathbf{P b}$ & La & $\mathrm{Ce}$ & Th \\
\hline 1 & 9 & $12 \mathrm{~S} / 12 \mathrm{E}-33 \mathrm{ACB}$ & 250 & RMCOS1250 & DF lava & 147 & 316 & 40 & 236 & 105 & 4 & 215 & 89 & 27 & 3.1 & 17 & 83 & 65 & 1 & 0 & 35 & 4 \\
\hline 2 & 9 & $12 \mathrm{~S} / 12 \mathrm{E}-33 \mathrm{ACB}$ & 280 & RMCOS 1280 & DF lava & 168 & 313 & 40 & 224 & 134 & 5 & 239 & 95 & 29 & 4.5 & 16 & 63 & 69 & 0 & 12 & 23 & 1 \\
\hline 3 & 9 & $12 \mathrm{~S} / 12 \mathrm{E}-33 \mathrm{ACB}$ & 415 & RMCOS1415 & DF-Pelton basalt & 137 & 237 & 36 & 293 & 342 & 7 & 349 & 126 & 34 & 9.4 & 20 & 125 & 115 & 0 & 19 & 35 & 4 \\
\hline 4 & 9 & $12 \mathrm{~S} / 12 \mathrm{E}-33 \mathrm{ACB}$ & 585 & LITOPAL585 & DF-Pelton basalt & 142 & 297 & 32 & 283 & 339 & 4 & 314 & 93 & 28 & 6.6 & 19 & 56 & 91 & 2 & 0 & 20 & 2 \\
\hline 5 & 9 & $12 \mathrm{~S} / 12 \mathrm{E}-33 \mathrm{ACB}$ & 725 & LITOPAL725 & DF-Pelton basalt & 160 & 302 & 37 & 285 & 274 & 5 & 352 & 88 & 26 & 6.0 & 22 & 81 & 91 & 1 & 13 & 26 & 2 \\
\hline 6 & 14 & 13S/12E-36BDB & $448-460$ & LITEA448460 & DF-Opal Springs basalt & 125 & 209 & 35 & 250 & 137 & 6 & 268 & 73 & 24 & 4.6 & 17 & 94 & 68 & 5 & 14 & 5 & 1 \\
\hline 7 & 54 & 15S/13E-20AAD & 520 & LITRED520 & DF lava & 106 & 213 & 35 & 226 & 174 & 6 & 255 & 102 & 28 & 7.5 & 19 & 69 & 64 & 3 & 9 & 24 & 1 \\
\hline 8 & 54 & 15S/13E-20AAD & 670 & LITRED670 & DF lava & 84 & 168 & 31 & 275 & 322 & 8 & 387 & 136 & 30 & 9.6 & 19 & 67 & 92 & 3 & 17 & 16 & 2 \\
\hline 9 & 54 & 15S/13E-20AAD & $775-780$ & LITRE775780 & DF lava & 217 & 382 & 38 & 259 & 119 & 3 & 219 & 87 & 25 & 5.2 & 16 & 73 & 73 & 2 & 0 & 25 & 2 \\
\hline 10 & 60 & 15S/14E-03BAA & $294-320$ & LITIS294320 & DF lava & 225 & 407 & 40 & 221 & 97 & 1 & 204 & 61 & 24 & 3.7 & 16 & 69 & 61 & 2 & 4 & 12 & 0 \\
\hline 11 & 61 & 15S/14E-14CDC & 279 & LITSM279 & DF lava & 148 & 287 & 42 & 233 & 111 & 2 & 240 & 65 & 24 & 4.3 & 18 & 92 & 66 & 0 & 0 & 9 & 1 \\
\hline 12 & 61 & 15S/14E-14CDC & $377-400$ & LITSM377400 & DF lava & 124 & 233 & 36 & 249 & 213 & 4 & 330 & 94 & 26 & 6.8 & 17 & 85 & 80 & 0 & 7 & 16 & 2 \\
\hline 13 & 64 & 15S/15E-23BBB & $240-250$ & RCPRTB240250 & DF lava & 136 & 243 & 39 & 331 & 418 & 7 & 313 & 100 & 30 & 6.8 & 19 & 76 & 100 & 0 & 16 & 10 & 1 \\
\hline 14 & 64 & $15 \mathrm{~S} / 15 \mathrm{E}-23 \mathrm{BBB}$ & $270-280$ & RCPRTB270280 & Prineville Basalt & 3 & 21 & 37 & 349 & 2,185 & 41 & 395 & 149 & 49 & 8.6 & 20 & 109 & 169 & 5 & 20 & 36 & 7 \\
\hline 15 & 64 & 15S/15E-23BBB & $370-380$ & RCPRTB 370380 & Prineville Basalt & 9 & 22 & 31 & 354 & 2,326 & 41 & 395 & 150 & 49 & 9.6 & 22 & 35 & 133 & 5 & 25 & 38 & 4 \\
\hline 16 & 66 & 16S/10E-13BBB2 & $218-254$ & RCNMN218254 & CR lava & 33 & 89 & 21 & 212 & 313 & 22 & 397 & 161 & 31 & 9.6 & 22 & 61 & 80 & 5 & 19 & 40 & 3 \\
\hline 17 & 70 & 16S/11E-18BAC & $347-360$ & RCMCT347360 & DF lava & 7 & 51 & 30 & 260 & 473 & 13 & 572 & 146 & 26 & 8.6 & 22 & 98 & 87 & 3 & 8 & 43 & 3 \\
\hline 18 & 71 & 16S/11E-33DDD & 195-203 & RCPT-195203 & DF lava & 0 & 8 & 19 & 284 & 455 & 18 & 483 & 158 & 34 & 10.8 & 25 & 40 & 106 & 3 & 12 & 52 & 5 \\
\hline 19 & 71 & 16S/11E-33DDD & 468 & RC-PT-468 & DF lava & 0 & 18 & 41 & 302 & 393 & 9 & 417 & 139 & 37 & 8.9 & 19 & 24 & 111 & 0 & 10 & 22 & 3 \\
\hline 20 & 83 & 16S/14E-17CDD & 332 & PB332 & DF lava & 176 & 315 & 44 & 238 & 61 & 2 & 144 & 46 & 22 & 2.1 & 14 & 91 & 51 & 0 & 1 & 14 & 0 \\
\hline 21 & 83 & $16 \mathrm{~S} / 14 \mathrm{E}-17 \mathrm{CDD}$ & 411 & PB411 & DF lava & 106 & 168 & 34 & 257 & 277 & 6 & 325 & 109 & 31 & 5.9 & 19 & 80 & 90 & 1 & 12 & 23 & 2 \\
\hline 22 & 84 & 16S/14E-21BBD & $437-478$ & LITMR437478 & DF lava & 140 & 198 & 42 & 238 & 125 & 1 & 230 & 70 & 25 & 4.2 & 16 & 85 & 62 & 0 & 14 & 20 & 1 \\
\hline 23 & 86 & 17S/11E-24DCA & 200 & RCAWG200 & DF lava & 66 & 76 & 23 & 236 & 433 & 11 & 536 & 135 & 24 & 8.4 & 20 & 96 & 89 & 5 & 8 & 38 & 2 \\
\hline 24 & 86 & 17S/11E-24DCA & 320 & RCAWG320 & DF lava & 80 & 75 & 26 & 237 & 400 & 9 & 549 & 140 & 26 & 8.8 & 22 & 76 & 100 & 3 & 20 & 45 & 4 \\
\hline 25 & 86 & 17S/11E-24DCA & $400-410$ & RCAWG400410 & DF lava & 25 & 66 & 27 & 222 & 464 & 10 & 649 & 166 & 30 & 14.1 & 19 & 61 & 96 & 4 & 26 & 39 & 3 \\
\hline 26 & 86 & 17S/11E-24DCA & $510-520$ & RCAWG510520 & DF lava & 29 & 66 & 22 & 216 & 473 & 12 & 639 & 160 & 28 & 13.9 & 20 & 49 & 93 & 3 & 22 & 63 & 3 \\
\hline 27 & 86 & 17S/11E-24DCA & $820-830$ & RCAWG820830 & DF lava & 38 & 75 & 30 & 205 & 429 & 14 & 592 & 149 & 28 & 11.9 & 20 & 64 & 86 & 4 & 16 & 53 & 3 \\
\hline 28 & 89 & 17S/12E-11DDB & $143-167$ & RCNLN143167 & NV lava & 153 & 357 & 22 & 197 & 290 & 7 & 344 & 114 & 24 & 6.5 & 14 & 52 & 81 & 1 & 14 & 35 & 0 \\
\hline 29 & 89 & 17S/12E-11DDB & 233-348 & RCNLN233348 & DF lava & 67 & 134 & 30 & 270 & 150 & 3 & 291 & 112 & 33 & 5.3 & 20 & 44 & 80 & 0 & 16 & 24 & 0 \\
\hline 30 & 89 & $17 \mathrm{~S} / 12 \mathrm{E}-11 \mathrm{DDB}$ & $348-412$ & RCNLN348412 & DF lava & 166 & 309 & 27 & 215 & 203 & 6 & 327 & 127 & 28 & 7.9 & 15 & 61 & 78 & 2 & 0 & 37 & 0 \\
\hline
\end{tabular}


Table 3. Trace element analyses of cuttings samples from selected wells in the upper Deschutes Basin, Oregon-Continued

Well location: Refer to "Well- and "Spring-Location System" for explanation. Sample depth: Given in feet below land surface. Stratigraphic Unit: DF, Deschutes Formation; CR, Cascade Range;

NV, Newberry Volcano.

\begin{tabular}{|c|c|c|c|c|c|c|c|c|c|c|c|c|c|c|c|c|c|c|c|c|c|c|}
\hline \multirow{2}{*}{$\begin{array}{l}\text { Sample } \\
\text { number }\end{array}$} & \multirow{2}{*}{$\begin{array}{c}\text { Well } \\
\text { number }\end{array}$} & \multirow[b]{2}{*}{ Well location } & \multirow{2}{*}{$\begin{array}{c}\text { Sample } \\
\text { depth }\end{array}$} & \multirow[b]{2}{*}{ Sample Name } & \multirow[b]{2}{*}{ Stratigraphic Unit } & \multicolumn{17}{|c|}{ Trace elements (in parts per million) } \\
\hline & & & & & & $\mathrm{Ni}$ & $\mathrm{Cr}$ & Sc & $\mathbf{V}$ & $\mathrm{Ba}$ & $\mathbf{R b}$ & Sr & $\mathrm{Zr}$ & $\mathbf{Y}$ & $\mathbf{N b}$ & Ga & Cu & Zn & $\mathbf{P b}$ & La & Ce & Th \\
\hline 31 & 89 & 17S/12E-11DDB & $465-505$ & RCNLN465505 & DF lava & 147 & 272 & 25 & 218 & 218 & 8 & 364 & 116 & 25 & 8.5 & 18 & 55 & 78 & 3 & 20 & 38 & 1 \\
\hline 32 & 89 & 17S/12E-11DDB & $517-520$ & RCNLN517520 & DF lava & 12 & 65 & 22 & 235 & 364 & 10 & 599 & 116 & 25 & 6.4 & 22 & 89 & 82 & 3 & 15 & 34 & 2 \\
\hline 33 & 91 & 17S/13E-20DAD & $96-125$ & RCBART96125 & NV lava & 145 & 327 & 27 & 211 & 299 & 6 & 342 & 122 & 26 & 7.6 & 18 & 44 & 78 & 0 & 8 & 30 & 0 \\
\hline 34 & 91 & 17S/13E-20DAD & $365-405$ & RCBAR365405 & DF lava & 134 & 250 & 29 & 247 & 229 & 8 & 391 & 109 & 26 & 6.8 & 17 & 67 & 77 & 2 & 0 & 30 & 2 \\
\hline 35 & 91 & 17S/13E-20DAD & $430-450$ & RCBAR430450 & DF lava & 8 & 50 & 29 & 264 & 370 & 11 & 407 & 132 & 37 & 8.2 & 22 & 44 & 99 & 3 & 3 & 37 & 1 \\
\hline 36 & 91 & 17S/13E-20DAD & $510-555$ & RCBAR510555 & DF lava & 115 & 228 & 32 & 241 & 257 & 7 & 331 & 116 & 29 & 8.4 & 15 & 56 & 79 & 4 & 0 & 19 & 0 \\
\hline 37 & 91 & 17S/13E-20DAD & $570-592$ & RCBAR570592 & DF lava & 138 & 239 & 31 & 236 & 257 & 6 & 324 & 113 & 28 & 7.4 & 19 & 58 & 88 & 1 & 1 & 16 & 0 \\
\hline 38 & 91 & 17S/13E-20DAD & $592-615$ & RCBAR592615 & DF lava & 135 & 246 & 30 & 256 & 237 & 3 & 321 & 114 & 32 & 8.4 & 17 & 87 & 89 & 1 & 0 & 27 & 1 \\
\hline 39 & 92 & 17S/14E-02CAA & $125-192$ & RCWOG125192 & DF lava & 185 & 316 & 38 & 225 & 25 & 1 & 143 & 49 & 22 & 2.5 & 12 & 102 & 48 & 1 & 11 & 0 & 0 \\
\hline 40 & 92 & 17S/14E-02CAA & $244-327$ & RCWOG244327 & DF lava & 80 & 173 & 33 & 308 & 356 & 4 & 314 & 119 & 32 & 8.6 & 17 & 82 & 100 & 1 & 19 & 15 & 1 \\
\hline 41 & 92 & 17S/14E-02CAA & $327-465$ & LITWO327465 & DF lava & 86 & 123 & 31 & 244 & 348 & 10 & 421 & 130 & 27 & 8.4 & 21 & 79 & 93 & 7 & 16 & 25 & 2 \\
\hline 42 & 93 & 17S/14E-11DCB & $342-530$ & LITRD342530 & DF lava & 103 & 168 & 36 & 273 & 312 & 5 & 327 & 112 & 30 & 8.5 & 17 & 77 & 94 & 0 & 12 & 22 & 2 \\
\hline 43 & 93 & 17S/14E-11DCB & $552-598$ & LITRD552598 & DF lava & 163 & 276 & 36 & 241 & 85 & 1 & 186 & 50 & 25 & 2.6 & 17 & 94 & 59 & 1 & 10 & 11 & 2 \\
\hline 44 & 93 & 17S/14E-11DCB & $655-675$ & LITRD655675 & DF lava & 159 & 243 & 32 & 204 & 142 & 4 & 202 & 58 & 23 & 2.7 & 14 & 83 & 55 & 1 & 9 & 27 & 2 \\
\hline 45 & 101 & 18S/12E-07DBD1 & 150 & RCCTB150 & CR lava & 53 & 82 & 25 & 249 & 474 & 31 & 307 & 174 & 36 & 12.9 & 18 & 53 & 95 & 7 & 9 & 42 & 4 \\
\hline 46 & 101 & 18S/12E-07DBD1 & 270 & RCCTB270 & CR lava & 19 & 79 & 21 & 257 & 245 & 10 & 491 & 117 & 24 & 6.9 & 21 & 80 & 82 & 1 & 7 & 49 & 1 \\
\hline 47 & 101 & 18S/12E-07DBD1 & 310 & RCCTB310 & CR lava & 17 & 83 & 20 & 243 & 256 & 10 & 489 & 117 & 25 & 6.3 & 20 & 86 & 77 & 1 & 20 & 27 & 2 \\
\hline 48 & 101 & 18S/12E-07DBD1 & 370 & RCCTB370 & CR lava & 33 & 96 & 19 & 263 & 322 & 11 & 475 & 134 & 29 & 6.5 & 22 & 89 & 93 & 2 & 14 & 32 & 4 \\
\hline 49 & 108 & 18S/13E-24DCB & $170-194$ & RCDB-170194 & NV lava & 168 & 379 & 33 & 199 & 144 & 9 & 234 & 77 & 23 & 4.1 & 16 & 61 & 64 & 1 & 0 & 22 & 2 \\
\hline 50 & 108 & 18S/13E-24DCB & 194-226 & RCDB-194226 & NV lava & 185 & 378 & 30 & 191 & 136 & 10 & 218 & 77 & 24 & 5.3 & 15 & 62 & 60 & 1 & 16 & 8 & 1 \\
\hline 51 & 108 & 18S/13E-24DCB & $226-275$ & RCDB-226275 & NV lava & 181 & 364 & 30 & 196 & 151 & 10 & 213 & 81 & 25 & 3.9 & 14 & 64 & 62 & 2 & 2 & 25 & 2 \\
\hline 52 & 108 & 18S/13E-24DCB & $294-352$ & RCDB294-352 & NV lava & 82 & 99 & 28 & 256 & 284 & 7 & 352 & 122 & 29 & 7.9 & 20 & 62 & 98 & 2 & 0 & 25 & 2 \\
\hline 53 & 108 & 18S/13E-24DCB & $352-405$ & RCDB352-405 & NV lava & 90 & 158 & 34 & 256 & 169 & 2 & 261 & 116 & 32 & 5.5 & 17 & 71 & 88 & 0 & 9 & 17 & 0 \\
\hline 54 & 108 & 18S/13E-24DCB & $424-521$ & RCDB424-451 & NV lava & 120 & 180 & 30 & 231 & 288 & 8 & 404 & 115 & 25 & 7.7 & 17 & 63 & 80 & 4 & 4 & 22 & 2 \\
\hline 55 & 108 & 18S/13E-24DCB & $536-632$ & RCDB536-632 & NV lava & 117 & 158 & 30 & 205 & 232 & 7 & 402 & 78 & 21 & 3.8 & 19 & 76 & 73 & 0 & 0 & 22 & 1 \\
\hline 56 & 108 & 18S/13E-24DCB & $710-721$ & RCDB710-721 & DF-equivalent lava & 30 & 96 & 31 & 207 & 345 & 21 & 412 & 141 & 28 & 10.3 & 20 & 59 & 78 & 4 & 20 & 30 & 2 \\
\hline 57 & 108 & 18S/13E-24DCB & $721-770$ & RCDB721-770 & DF-equivalent lava & 33 & 105 & 37 & 192 & 303 & 19 & 409 & 135 & 28 & 10.1 & 19 & 53 & 74 & 0 & 19 & 25 & 3 \\
\hline 58 & 108 & 18S/13E-24DCB & $770-785$ & RCDB770-785 & DF-equivalent lava & 47 & 122 & 26 & 189 & 285 & 20 & 391 & 127 & 26 & 9.6 & 18 & 64 & 70 & 3 & 16 & 33 & 2 \\
\hline 59 & 108 & 18S/13E-24DCB & 785-904 & RCDB785-904 & DF-equivalent lava & 50 & 126 & 28 & 199 & 290 & 19 & 376 & 123 & 25 & 9.2 & 16 & 52 & 71 & 5 & 8 & 35 & 3 \\
\hline
\end{tabular}




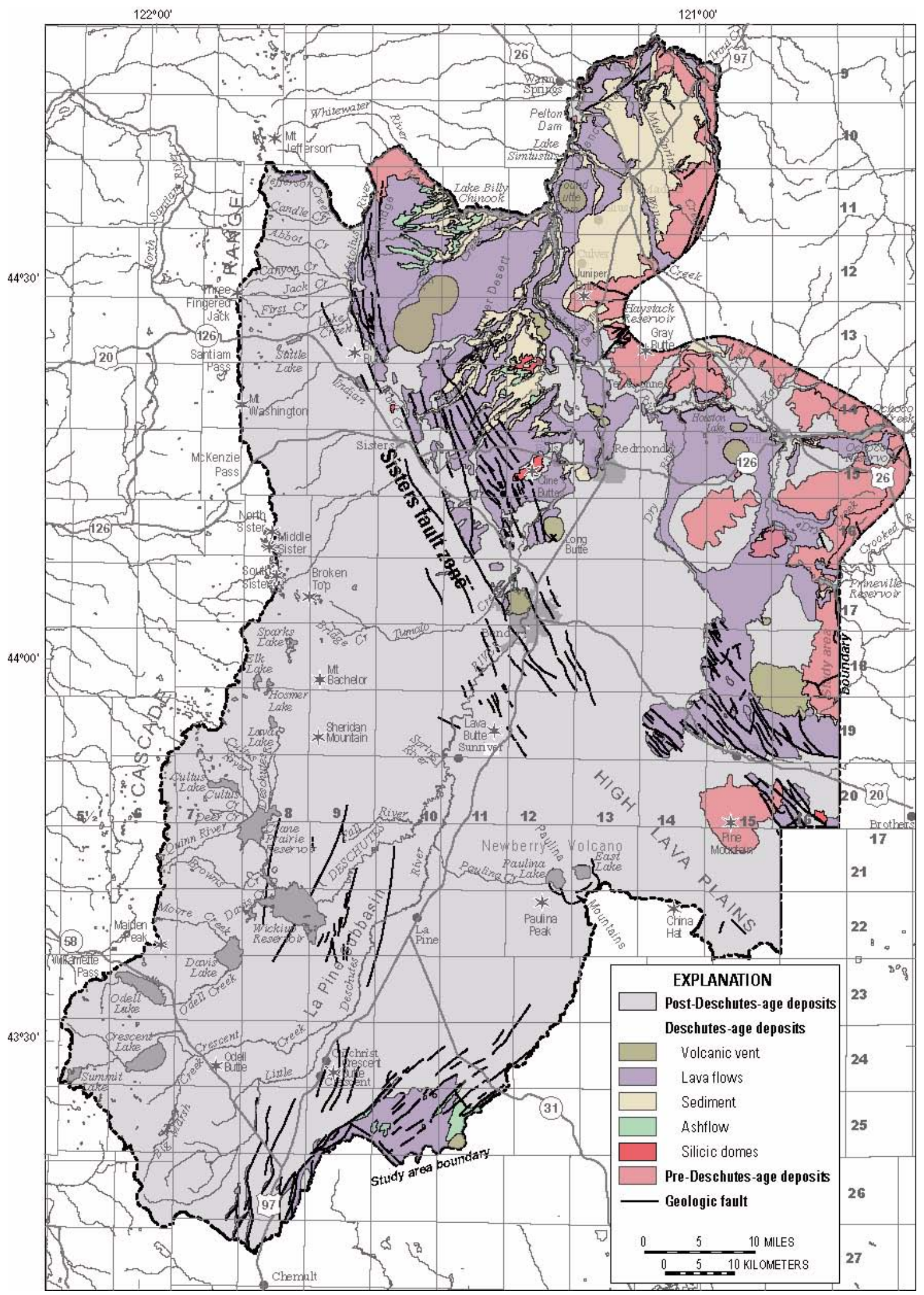

Figure 14. Extent of surface exposures of the Deschutes Formation and age-equivalent deposits. 
The deposits reflect the clay-rich and silicic nature of their sources. This is apparent on a natural gamma log (fig. 15) for well 11S/13E-24BCD1 (well no. 6, plate 1) near Madras, where sediment with a high gamma signature is interbedded with contrasting low-gamma basalt flows, all within the Deschutes Formation. Well yields within the inactive-margin deposits are typically less than $30 \mathrm{gal} / \mathrm{min}$ but, in a few cases, are as large as $300 \mathrm{gal} / \mathrm{min}$. The larger yields are likely due to interbedded lava flows or locally coarse-grained sedimentary beds.

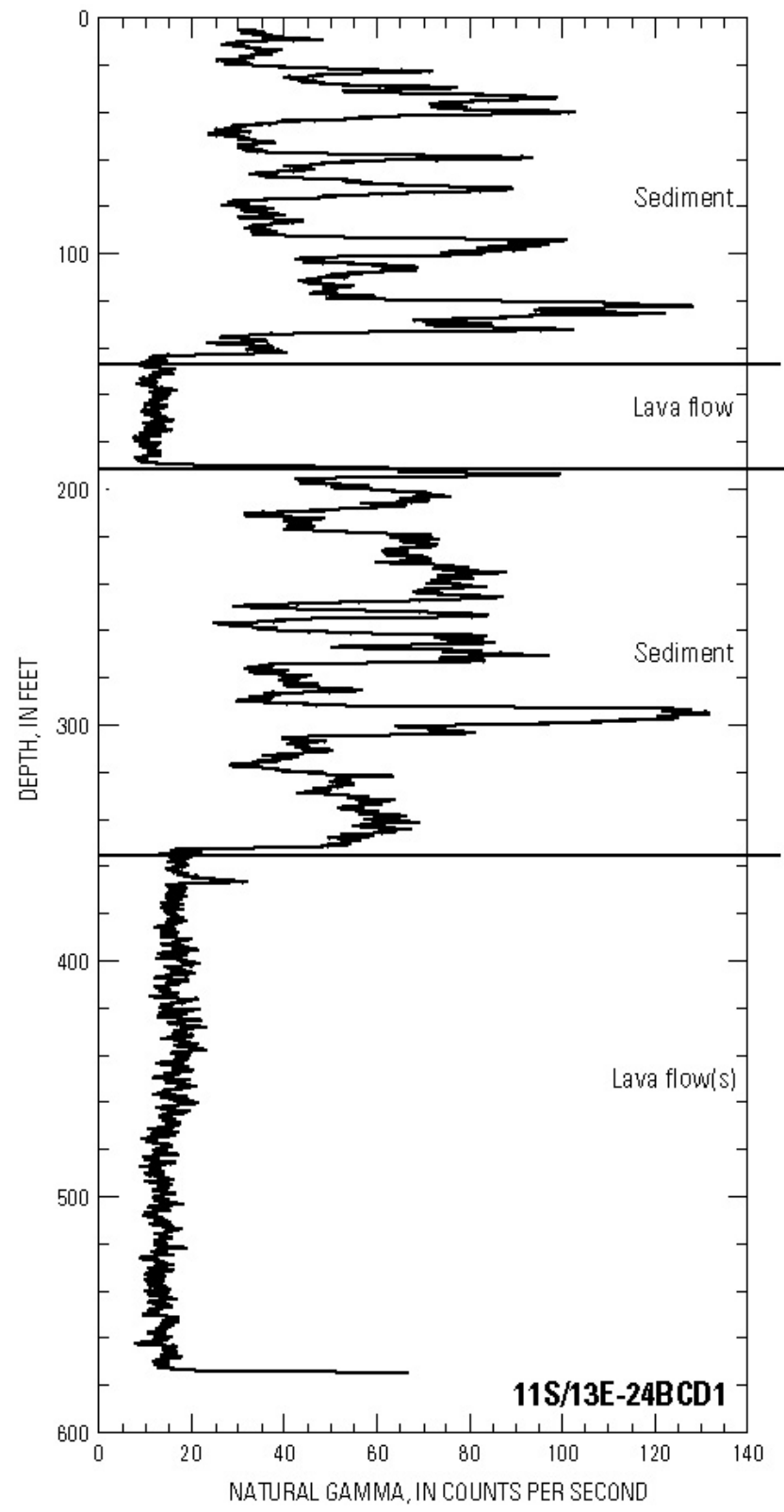

Figure 15. Natural gamma log for a well constructed into the inactive-margin facies of the Deschutes Formation near Madras, Oregon (11S/13E-24BCD1, well no. 6, plate 1).
An aquifer test was conducted at one of the higher-yielding inactive-margin wells during this study. A sand-and-gravel aquifer within the inactivemargin facies was tested at well 11S/13E-01BCA2 (well no. 4, plate 1) in Madras. The aquifer test yielded hydraulic conductivity estimates of 110 to $160 \mathrm{ft} / \mathrm{d}$ for the unit (Gannett and others, 2001).

\section{Arc-Adjacent Alluvial-Plain Facies}

The arc-adjacent alluvial plain represents a variety of volcanic and volcaniclastic materials that erupted or were otherwise shed from the flanks of active volcanoes approximately 7.5 million years to 4.0 million years ago. The deposits within the arc-adjacent plain include lava flows, volcanic vent material, ashflows, ash-fallout, and a variety of volcanically derived sediment. The variability of the material encountered within the arc-adjacent alluvial-plain region is illustrated in figure 16A. Figure 16A shows a natural gamma log of well 14S/11E-18BDB (well no. 24, plate 1) near Henkle Butte that has been annotated with material descriptions on the basis of microscopic examination of drill cuttings.

The sedimentary and pyroclastic material within the arc-adjacent alluvial-plain region tends to be relatively coarse-grained because it is near the volcanic source areas. The topographic slope and ongoing drainage development in the source areas resulted in preferential orientation of subsequent lava flows and ash-flows that followed shallow paleodrainages towards the east and northeast (Smith, 1986b). The east to northeast orientation of the units probably results in many local preferential ground-water flow paths in those directions.

Two notable intrabasinal silicic volcanic vents are also found in the arc-adjacent alluvial-plain region. They are the rhyolite and rhyodacite volcanic dome complexes that form Cline Buttes and small hills near Steelhead Falls. The dome complexes have been included in the Deschutes Formation on the basis of radiometric ages (Sherrod and others, in press). Geologic mapping in the Steelhead Falls area has shown that Deschutes Formation ash-flow and sedimentary deposits on-lap the dome rocks (Ferns and others, 1996b). That relation is subptantiated by well data and the natural gamma log for well $14 \mathrm{~S} / 11 \mathrm{E}-$ 01DDD1 (well ho. 22, plate 1) shown in figure 16B.

The hydrdlogic character of the arc-adjacent material is as variables that form it. 

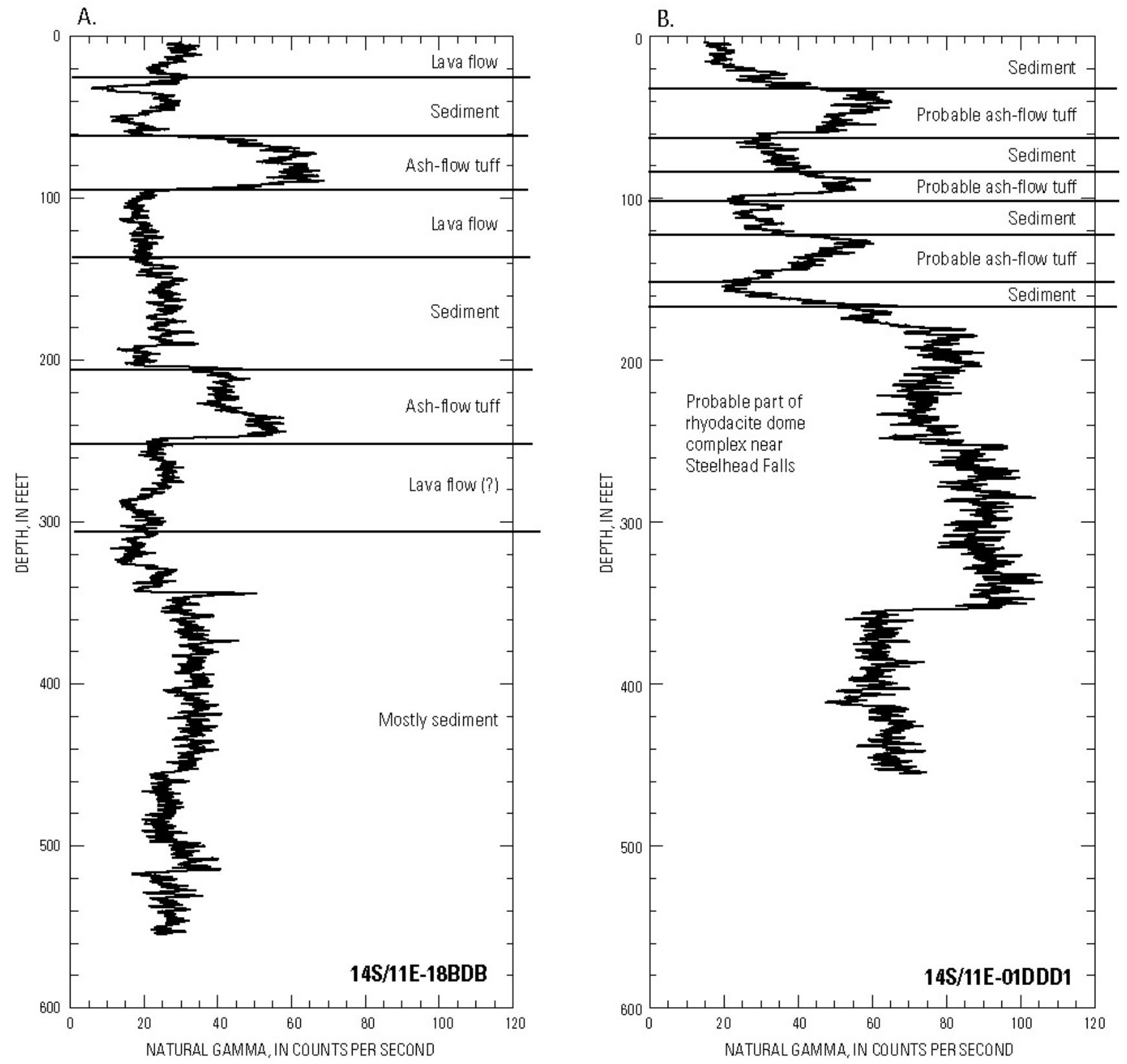

Figure 16. Natural gamma logs for wells constructed into the arc-adjacent alluvial-plain facies of the Deschutes Formation. A. Well near Henkle Butte (14S/11E-18BDB, well no. 24, plate 1); B. Well near Steelhead Falls (14S/11E-01DDD1, well no. 22, plate 1).

The coarse-grained sedimentary strata and the fractured and rubbly character of thin intracanyon lava flows result in well yields of hundreds of gallons per minute. Hydraulic conductivity estimates of 1.5 to $2.3 \times 10^{3} \mathrm{ft} / \mathrm{d}$ were derived from an aquifer test conducted near the Redmond airport (Gannett and others, 2001). The well (15S/13E-22CBA2; well no. 57, plate 1) is open to sand, gravel, and lava deposited in the arc-adjacent plain.

The fractured nature of the breccia associated with rhyolitic domes within the arc-adjacent facies has resulted in some relatively high-yielding wells adjacent to Cline Buttes and near Steelhead Falls. The high permeability of the fractured dome complex near Steelhead Falls also contributes a substantial amount of flow, about $150 \mathrm{ft}^{3} / \mathrm{s}$, to the Deschutes River (Ferns and others, 1996a).

\section{Proximal Facies}

Proximal facies deposits account for most of the geologic material encountered in the Deschutes Formation west of Squaw Back Ridge in the northwestern 
part of the study area, and south and east of Long Butte in the southeastern part of the study area. The rocks represent deposition that occurred relatively close to their volcanic vents and typically consist of relatively thin ( $<50 \mathrm{ft}$ thick) lava flows with interbedded volcanic flow breccia and cinders.

Interbedded pyroclastic material and sedimentary units occur locally within the proximal deposits. On the basis of well data, the interbedded sedimentary and pyroclastic deposits are notably abundant within the proximal deposits near the contact with the John Day Formation. Within the proximal facies rocks in the southeastern part of the study area, interbedded sedimentary and pyroclastic deposits appear more prevalent in an alignment roughly corresponding with the present-day Dry River. Those interbedded deposits probably define paleochannels of an ancestral drainage. The natural gamma log for well 17S/14E-02CAA (well no. 92, plate 1), located adjacent to the southwestern flank of Powell Buttes, shows the interbedded nature of the Deschutes Formation at that locale (fig. 17).

Public supply wells in the Bend area are good examples of the yield potential from layered lava flows within the proximal facies. Yields from large-diameter (greater than 10 inches) wells near Bend range from about $400 \mathrm{gal} / \mathrm{min}$ to $2,000 \mathrm{gal} / \mathrm{min}$. An aquifer test conducted for the City of Bend at well 18S/12E05BDB (well no. 99, plate 1) yielded a hydraulic conductivity estimate of $1.5 \times 10^{2} \mathrm{ft} / \mathrm{d}$ (Gannett and others, 2001). The well is open to lava and tuff.

\section{Ancestral Deschutes River Facies}

The ancestral Deschutes River facies consists predominately of coarse sandstone and conglomerate streambed deposits alternating with fine sandstone and mudstone overbank flood deposits, a variety of volcaniclastic sediment, and distal parts of many ash-flow tuffs and lava flows. Smith (1986b, 1987) described the ancestral Deschutes River deposits as occurring within a relatively narrow area from Redmond to Gateway that corresponds roughly to the present path of the Crooked and Deschutes Rivers (fig. 7).

Some of the geologic materials along the paleochannels are exceptionally permeable, as shown by the enormous quantity (approximately $1,500 \mathrm{ft}^{3} / \mathrm{s}$ ) of ground water that discharges to the Crooked and Deschutes Rivers in that region (Stearns, 1931; Sceva, 1968; Gannett and others, 2001). Ground water discharges to the streams mainly through conglomerate

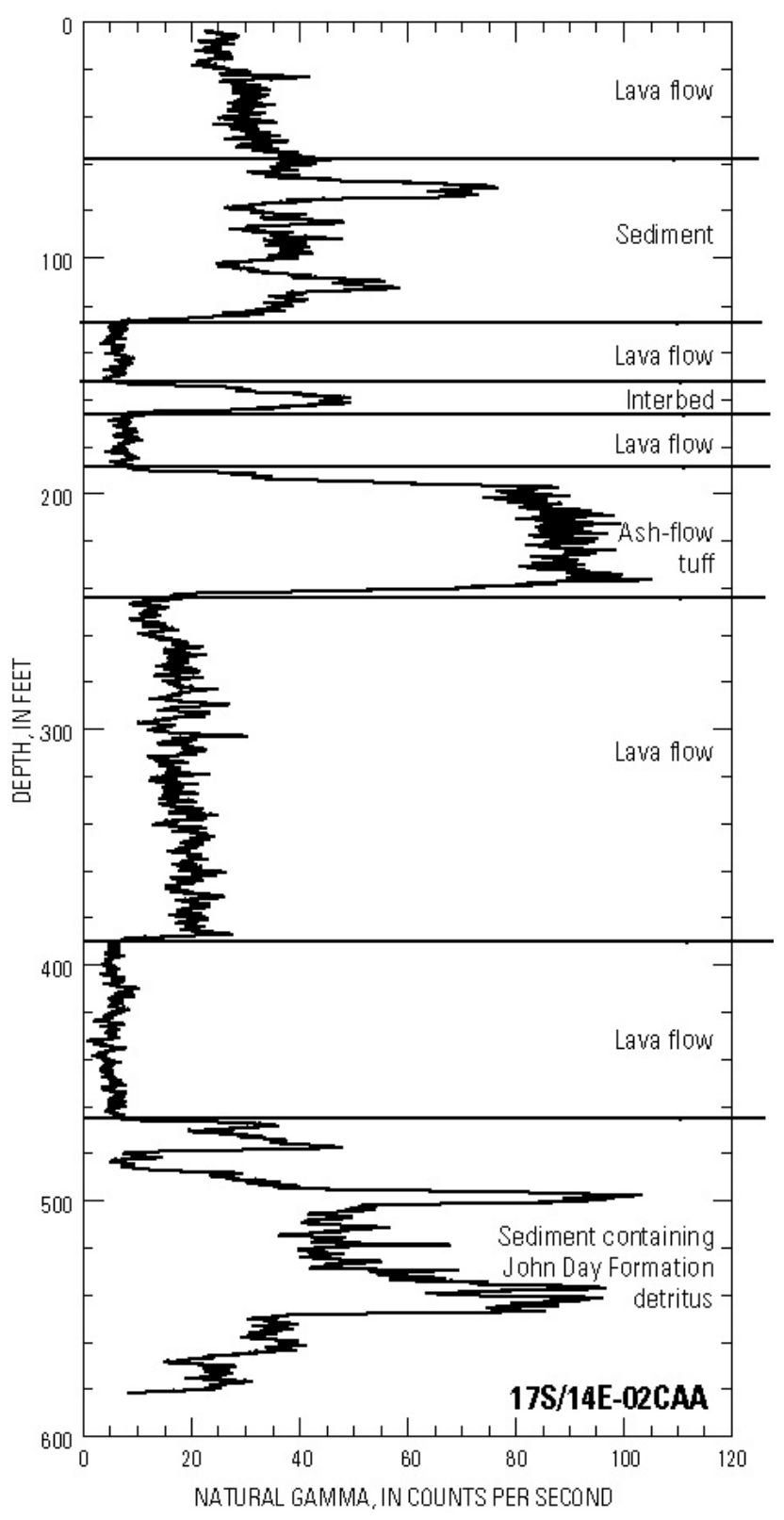

Figure 17. Natural gamma log for a well constructed into layered lava flows within the Deschutes Formation southwest of Powell Buttes, upper Deschutes Basin, Oregon (17S/14E-02CAA, well no. 92 , plate 1).

and fractured intracanyon lava flows (Ferns and others, 1996a). As noted by Stearns (1931), most of the ground water that actually discharges to the Crooked River is principally from two intracanyon lava flow sequences within the Deschutes Formation: the Pelton basalt and the Opal Springs basalt.

The Pelton basalt is 7.42 million years old and the oldest exposed volcanic unit within the Deschutes Formation (Smith, 1986b). The unit consists of several lava flows that may have a total thickness of nearly $400 \mathrm{ft}$. 
The Pelton basalt forms part of the abutments for Round Butte Dam and was the subject of intense analysis prior to construction of the dam. The thickness of the Pelton basalt at Round Butte Dam is $260 \mathrm{ft}$ (Bechtel Corporation, 1958). Individual flows within the Pelton basalt have been described and analyzed for major oxides and some trace elements by Jay (1982) and Smith (1986b). Relatively low aluminum oxide $\left(\mathrm{Al}_{2} \mathrm{O}_{3}\right)$ (average 15.8 weight percent) from the Pelton lava flows led Smith (1986b) to speculate that the flows may have originated from southeast of the basin, where basalt tends to exhibit lower (less than 16.0 percent) $\mathrm{Al}_{2} \mathrm{O}_{3}$ values compared to lava flows from Cascade volcanoes. Indeed, lava flows in several wells in the southeastern part of the study area that were sampled and analyzed during this study exhibit rock chemistry similar to the Pelton basalt (fig. 3, tables 2 and 3). This implies that an ancestral Deschutes River and tributary may have existed both northeast and southwest of Powell Buttes, and that a possible source area for the lava flows may be in the West Butte-Bear Creek Buttes area. Unfortunately, many of the lava flows occur above the saturated zone in those areas, so they are not potential sources of ground water.

The thickest sequence of Pelton basalt appears to be a few hundred feet below land surface in the vicinity of Opal Springs. A water well drilled at Opal Springs for the Deschutes Valley Water Company penetrated a 376-ft-thick sequence of Pelton basalt, first encountered at a depth of $364 \mathrm{ft}$ below the canyon floor. The identity of Pelton basalt in the well is based on elevation, thickness, appearance (video tape from a downhole camera), and rock chemistry (tables 2 and 3 ). The Opal Springs well \#1 (12S/12E-33ACB, well no. 9, plate 1) is a flowing well that yields over $4,000 \mathrm{gal} / \mathrm{min}$ from an interflow zone near the bottom of the basalt sequence (Steve Bruce, David J. Newton Associates, personal commun., 1998). A thick sequence of lava flows within a now-abandoned well at the Opal City site (Stearns, 1931) reportedly occurs at a similar elevation as the basalt sequence in the Opal Springs well and is probably also Pelton basalt.

The Opal Springs basalt is a sequence of two to four lava flows with interbedded paleosols and tuff that are exposed in the Crooked River canyon from above Osborne Canyon to Opal Springs (Ferns and others, 1996b). Exposures of the Opal Springs basalt, like the Pelton basalt, probably define a paleochannel of the ancestral Deschutes River. The Opal Springs basalt is up to $120 \mathrm{ft}$ thick. A flow low in the sequence has a radiometric age of $5.77 \mathrm{Ma}$ (Smith, 1986b).

The Opal Springs basalt is very permeable. Over $1,000 \mathrm{ft}^{3} / \mathrm{s}$ of ground water discharges from Opal Springs lava exposed in the Crooked River canyon between Osborne Canyon and Opal Springs (Ferns and others, 1996a; Gannett and others, 2001).

\section{Deposits of the Cascade Range and Newberry Volcano}

Most of what is known about the hydrogeology of the Cascade Range and Newberry Volcano is based on well data from the eastern margin of the Cascade Range and from wells penetrating lava flows on the flanks of Newberry Volcano. Some information on conditions at higher elevations in the Cascade Range and at Newberry Volcano within the study area has been obtained from a few deep geothermal exploration wells, two deep (800- to $1000-\mathrm{ft}$ ) water wells, and several shallow water wells.

The youthful and fractured character of the Cascade Range deposits results in rapid infiltration of precipitation and snowmelt, as shown by the lack of drainage development on the east side of the Cascade Range. The Cascade Range volcanic units provide the primary pathway for most of the ground-water recharge that occurs within the study area. Large rates of canal leakage (Gannett and others, 2001) into the Newberry Volcano lava flows near Bend demonstrate the open-fracture character for those deposits as well.

The Tumalo fault and Green Ridge fault zone generally mark the basinward limit of Quaternary Cascade Range deposits. However, some Cascade Range lava and pyroclastic flows have filled drainages that breached escarpments formed by the Tumalo fault and other strands of the Sisters fault zone between Bend and Sisters (fig. 18).

Lava flows from Newberry Volcano overlie most other geologic units east of the Tumalo fault near Bend. Natural gamma logs of wells penetrating the Newberry flows near Bend reveal a remarkably constant gamma count throughout the entire thickness of the sequence (fig. 19A). That characteristic makes the gamma log a potentially useful tool for distinguishing the Newberry flows from other underlying lava units. The Newberry flows thin to the north away from the volcano (see cross-section B in MacLeod and others, 1995). 


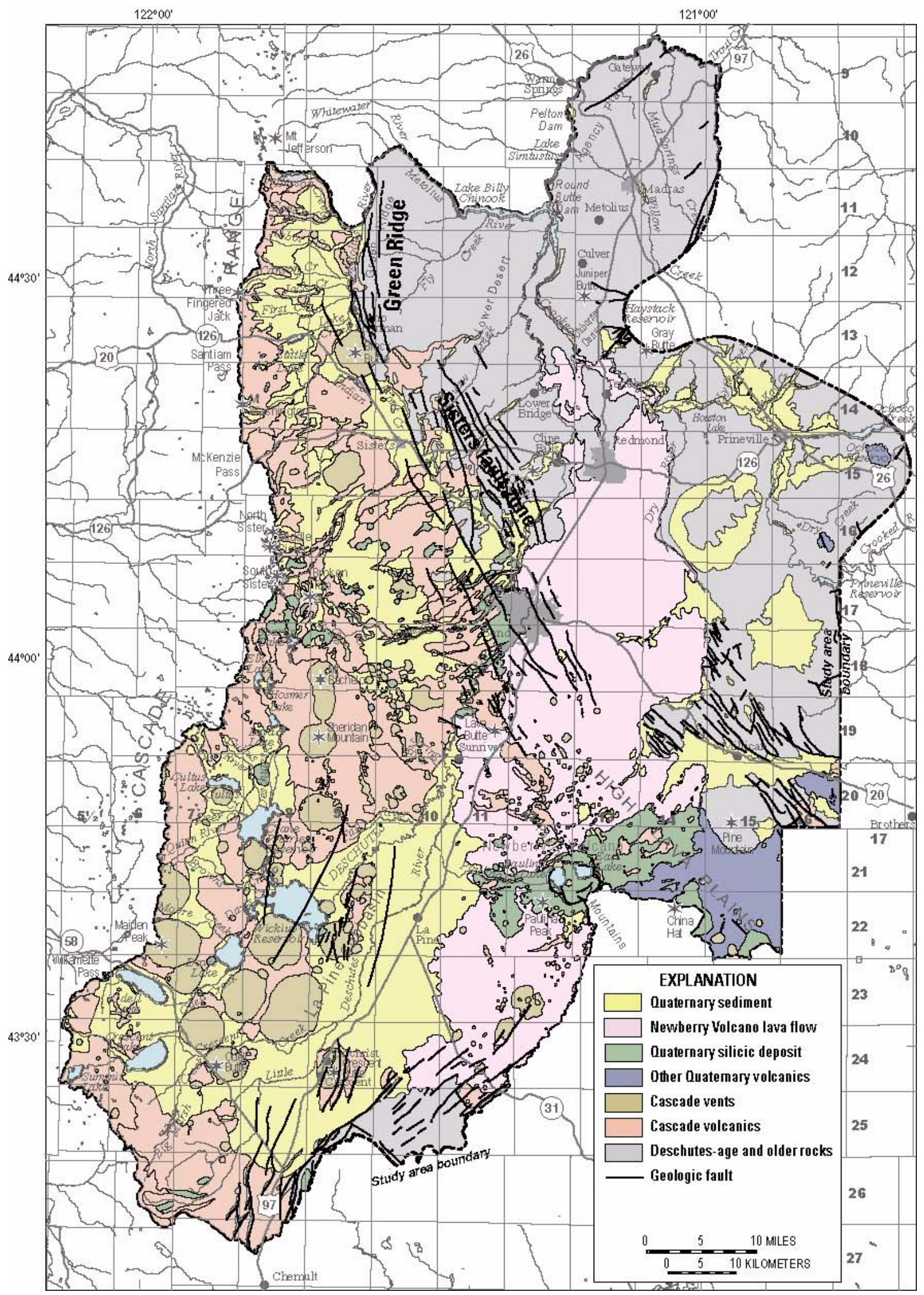

Figure 18. Extent of deposits of the Cascade Range and Newberry Volcano. 
A.

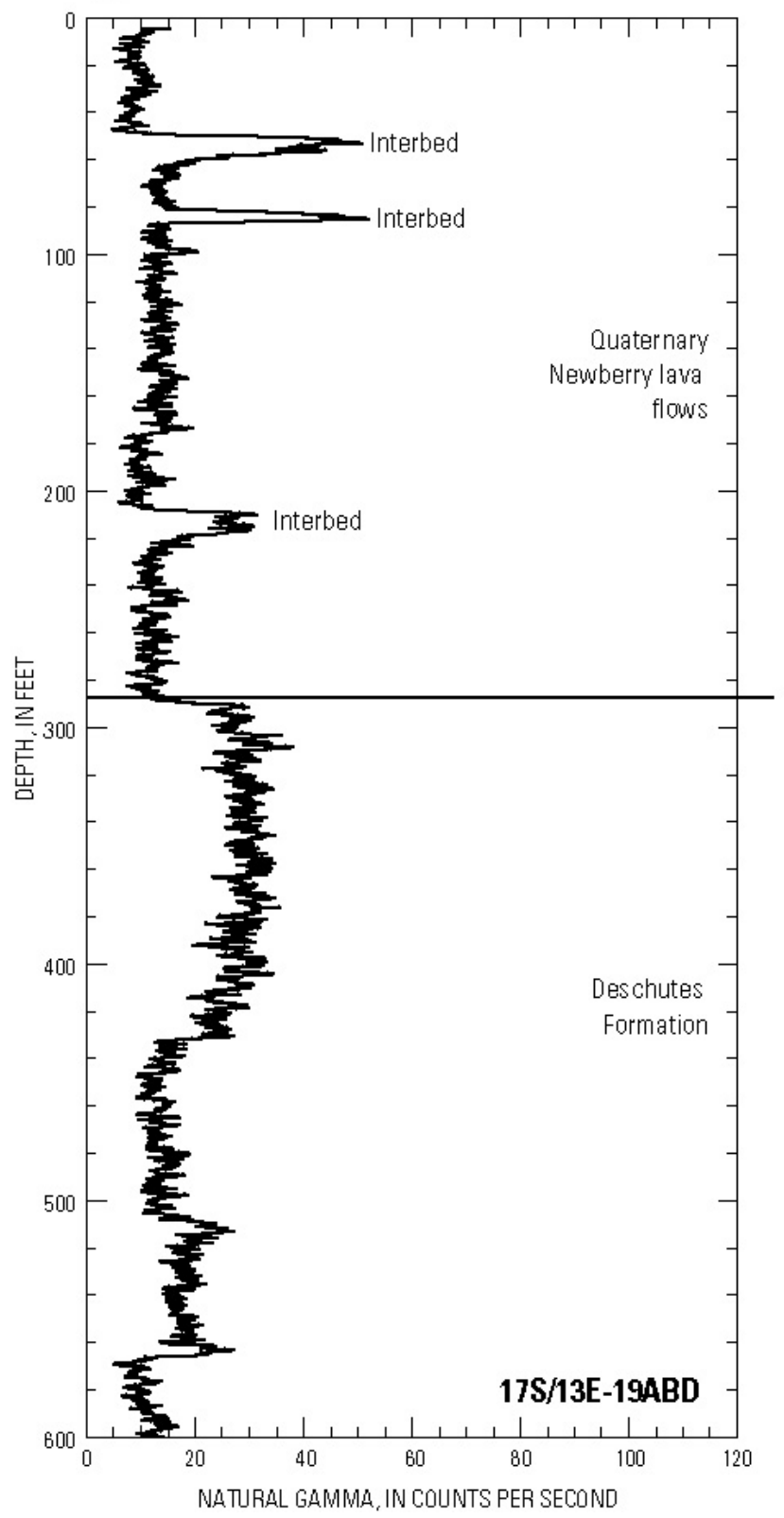

B.

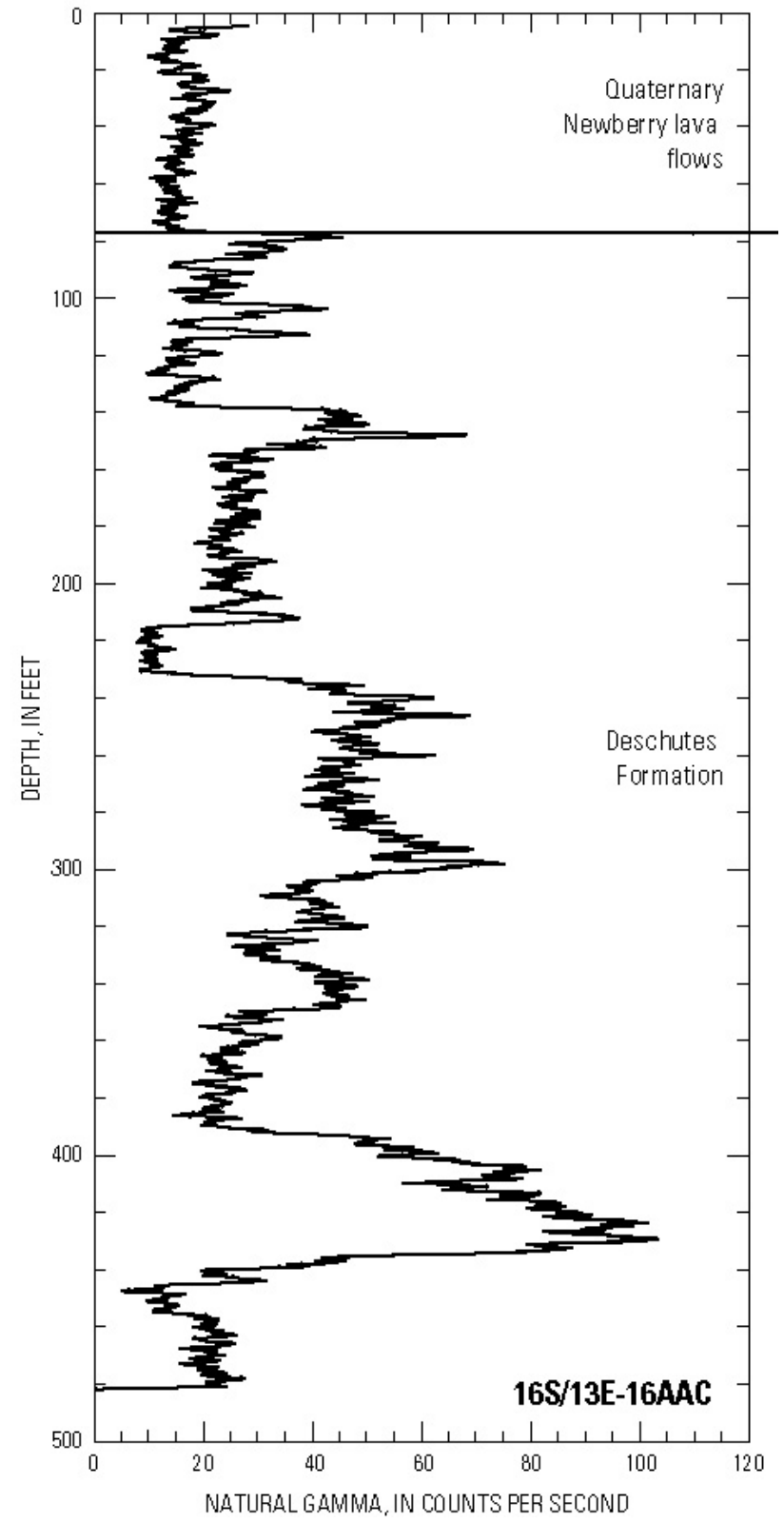

Figure 19. Natural gamma logs for wells penetrating Quaternary lava flows from Newberry Volcano and underlying Deschutes Formation strata. A. Well near Bend airport (17S/13E-19ABD, well no. 90, plate 1); B. Well 5 miles east-northeast of Long Butte (16S/13E-16AAC, well no. 81, plate 1).

On the basis of natural gamma logs and geologic material descriptions, it appears that the flows thin from about $285 \mathrm{ft}$ thick near the Bend Airport in well 17S/13E-19ABD (well no. 90, plate 1) (fig. 19A) to approximately $75 \mathrm{ft}$ thick in well $16 \mathrm{~S} / 13 \mathrm{E}-16 \mathrm{AAC}$ (well no. 81, plate 1) (fig. 19B) located 5 miles east-northeast from Long Butte, a distance of about 7 miles. The Newberry flows serve as conduits for canal leakage and other incidental recharge but are only locally saturated north and east of Bend.
West of the Sisters fault zone and south of Bend, the Cascade Range and Newberry deposits are saturated and are an important source of ground water. Numerous wells have been drilled into those deposits. In addition, Cascade Range deposits provide water to many spring-fed streams. In fact, approximately $2,600 \mathrm{ft}^{3} / \mathrm{s}$ (or about one-half the total basin discharge) of stream flow is derived from springs discharging from Cascade Range deposits (Gannett and others, 2001). Many of the springs within the Cascade Range 
units are associated with lava flows. Paulina Springs near the head of Indian Ford Creek and springs at the head of Fall River are good examples. Paulina Springs (which discharges about $10 \mathrm{ft}^{3} / \mathrm{s}$ ) is one of several springs that discharge from the toe of a Cascade Range lava flow. The head of Fall River (nearly $100 \mathrm{ft}^{3} / \mathrm{s}$ ) appears to issue near the boundary of a young drainage filled by lava flows.

Well yields from the few deep water wells developed in the Cascade Range deposits are relatively large. The yields range from a couple of hundred gallons per minute to nearly $2,000 \mathrm{gal} / \mathrm{min}$. For example, one of the wells at Mount Bachelor yields $1,850 \mathrm{gal} / \mathrm{min}$.

Permeability measurements in rocks of the Cascade Range and Newberry Volcano are sparse and limited to relatively shallow depth. Two aquifer tests conducted in Cascade Range deposits have yielded estimates of hydraulic conductivity ranging from 9 to $600 \mathrm{ft} / \mathrm{d}$ (Gannett and others, 2001). The $9 \mathrm{ft} / \mathrm{d}$ value is from well 18S/09E-20BDA (well no. 95, plate 1) at Mount Bachelor, and is open to cinders. The $600 \mathrm{ft} / \mathrm{d}$ value is from well 14S/10E-30DDB2 (well no. 20, plate 1) located near Sisters, and open to lava.

There are no permeability measurements below several hundred feet in the Cascade Range or on Newberry Volcano. Knowledge about permeability distribution at depth is inferred from geothermal gradient measurements and heat-flow studies. In simulating ground-water flow and heat transport in the Cascade Range, Ingebritsen and others (1992) estimated the permeability of rocks younger than $2.3 \mathrm{Ma}$ to be about $10^{-14} \mathrm{~m}^{2}$ (square meters), which is equivalent to a hydraulic conductivity of about $0.018 \mathrm{ft} / \mathrm{d}$ assuming a water temperature of $5^{\circ} \mathrm{C}$. The permeability of rocks with ages between 4 and $8 \mathrm{Ma}$ was estimated to be $5.0 \times 10^{-16} \mathrm{~m}^{2}$ which is equivalent to a hydraulic conductivity of about $9.1 \times 10^{-4} \mathrm{ft} / \mathrm{d}$. Ingebritsen and others (1992) found that higher near-surface permeability, on the order of $10^{-14}$ to $10^{-12} \mathrm{~m}^{2}(.018$ to $1.8 \mathrm{ft} / \mathrm{d})$, was required in their simulation to match ground-water recharge estimates.

Blackwell and Priest (1996) suggest that the heat transfer pattern in the Cascade Range is predominantly conductive, indicating that the permeability in all but the youngest rocks below a depth of 300 to $900 \mathrm{ft}$ is orders of magnitude lower than the estimates of Ingebritsen and others (1992). They also suggest that ground-water flow at velocities sufficient to affect heat flow in the high Cascade Range is restricted to local regions, such as high-permeability fault zones, except in the 0 to $1,500 \mathrm{ft}$ depth range. The volume of water moving through the low-permeability strata at depths greater than 1,500 ft in the Cascade Range is sufficiently small to be considered negligible compared to the overall ground-water budget, and these lowpermeability strata are for all practical considerations the base of the regional ground-water flow system. Temperature gradient data (Swanberg and others, 1988) and hydrothermal mineralization data (Keith and others, 1986) suggest that a similar loss of permeability occurs at depth beneath Newberry Volcano.

Mathematical modeling of ground-water discharge to spring-fed streams in the Cascade Range by Manga $(1996,1997)$ yielded permeability values for near-surface rocks less than about 2.0 Ma of about $10^{-11} \mathrm{~m}^{2}$, which equates to a hydraulic conductivity of about $18 \mathrm{ft} / \mathrm{d}$ assuming a water temperature of $5^{\circ} \mathrm{C}$. This estimate is an order of magnitude larger than the upper value of Ingebritsen and others (1992) for nearsurface rocks, where most of the ground-water flow occurs. The permeability estimates of Manga (1996, 1997) and Ingebritsen and others (1992) are considered to be a reasonable range of values for the younger, near-surface strata in the Cascade Range.

The depth beneath the Cascade Range and Newberry Volcano at which permeability is greatly reduced due to hydrothermal alteration and secondary mineralization varies from place to place and is poorly constrained. The overall geometry of this boundary surface is not well known. Data from geothermal test wells indicate that the transition occurs at depths ranging from 300 to $1,200 \mathrm{ft}$ and that primary permeability can be assumed to be virtually absent below $1,500 \mathrm{ft}$ (Blackwell and others, 1990; Ingebritsen and others, 1992; Blackwell and Priest, 1996).

\section{Quaternary Sedimentary Deposits}

Numerous relatively small, shallow, water-bearing zones exist within Quaternary sediment located throughout the study area (fig. 20). Those localized aquifers provide an important source of water to communities such as Sisters, Prineville, La Pine, and Camp Sherman.

Relatively coarse-grained glacial outwash deposits (included in unit Qs on fig. 11 and plate 1) are the principal water-bearing units in the vicinity of Sisters and Camp Sherman. Wells constructed into those deposits and interbedded Pleistocene lava flows have yields ranging from 30 to $2,000 \mathrm{gal} / \mathrm{min}$. 


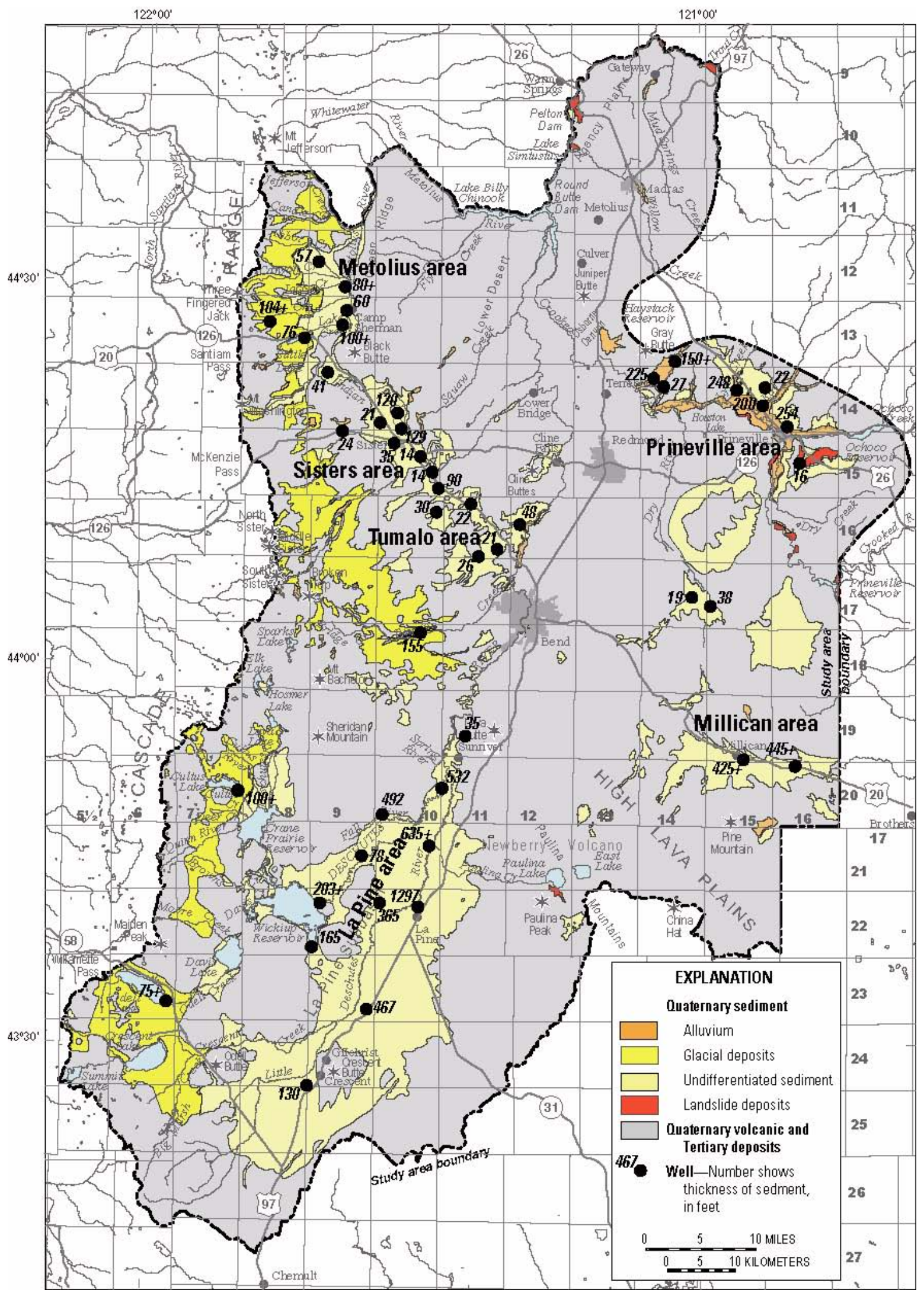

Figure 20. Distribution and thickness of Quaternary sediment. 
An aquifer test conducted on well $15 \mathrm{~S} / 10 \mathrm{E}-$ 05BBB (well no. 41, plate 1) near Sisters yielded a hydraulic conductivity estimate of $100 \mathrm{ft} / \mathrm{d}$ (Gannett and others, 2001). The well is open to glacial outwash and interbedded lava.

The Tumalo fault escarpment near Sisters appears to have provided an elongate depositional center for the thickest accumulation of the sediment in that area (fig. 11). The outwash sediment is locally over $100 \mathrm{ft}$ thick in the Sisters area. A natural gamma

A.

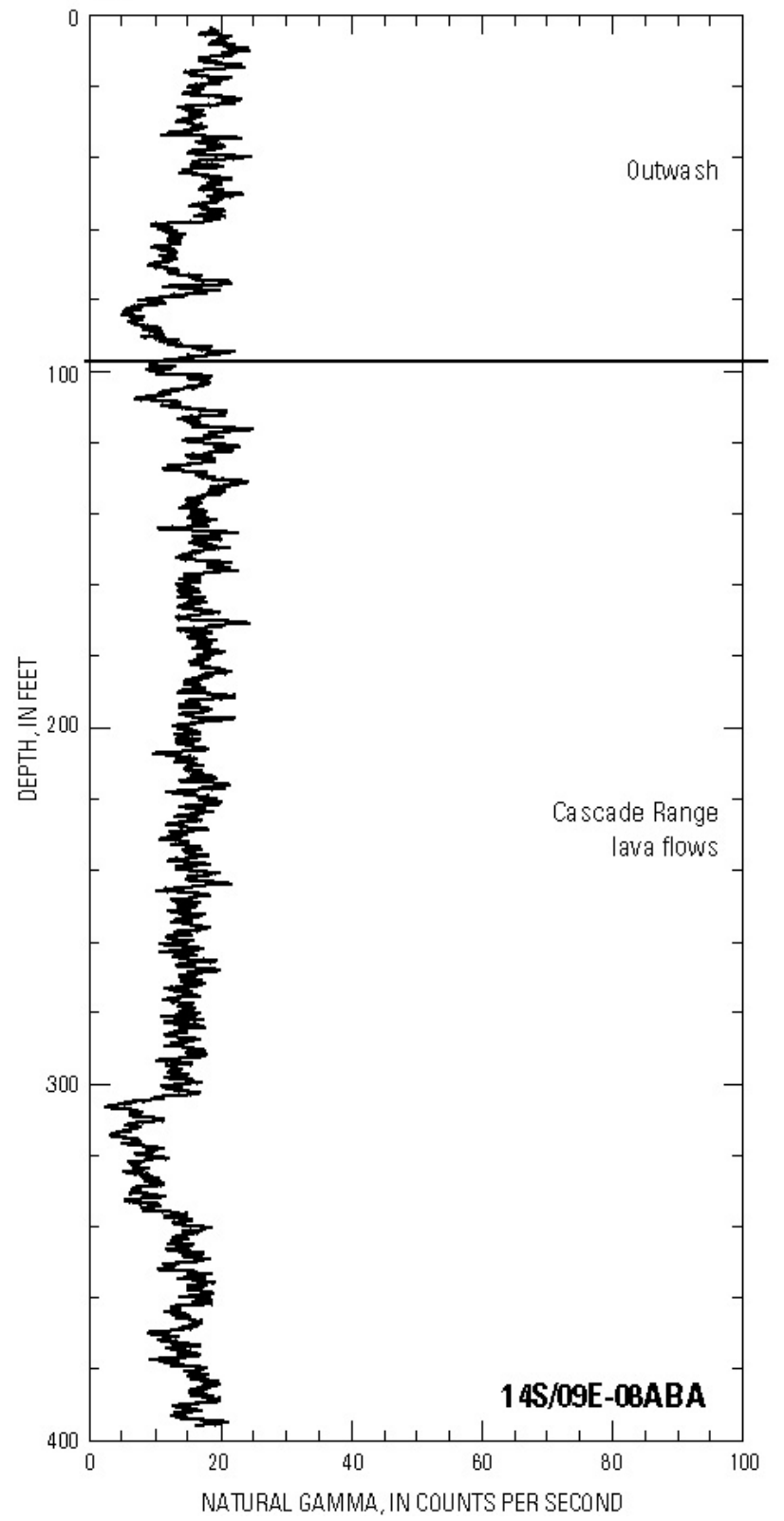

log for a well near Black Butte (14S/09E-08ABA) (well no. 16, plate 1) that penetrates outwash deposits and underlying Cascade Range lava flows shows an unremarkable gamma trace (fig. 21A). The similarity of the gamma signature in the outwash deposit and the underlying lava flows results from the similarity of the sedimentary material (mostly mafic volcanic debris) and that of the underlying lava. A natural gamma log for a well near Sisters (14S/10E-28DDC2) (well no. 18, plate 1) that is completed only into outwash deposits shows a similar gamma trace (fig. 21B).

B.

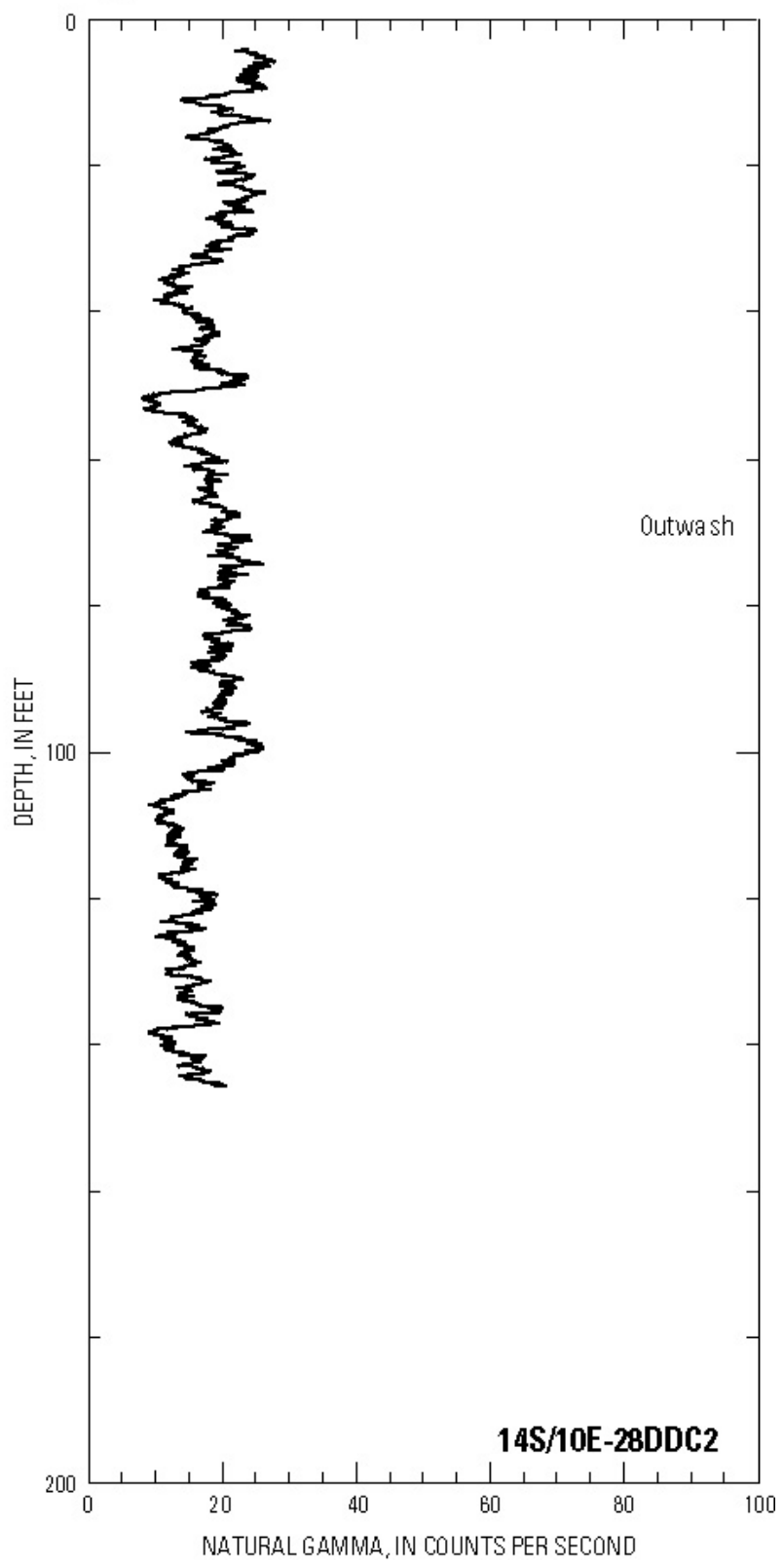

Figure 21. Natural gamma logs for wells penetrating Quaternary outwash deposits and lava flows. A. Well near Black Butte (14S/09E-08ABA, well no.16, plate 1); B. Well near Sisters (14S/10E-28DDC2, well no. 18, plate 1). 
Outwash deposits near Camp Sherman are also locally more than $100 \mathrm{ft}$ thick. Subsurface data are too sparse in the upper Metolius River area to construct a meaningful thickness map of the outwash deposits and thus determine the geometry of the unit. However, the Green Ridge escarpment and its associated downdropped blocks formed a boundary to east-draining streams that probably resulted in a north-trending depositional center for the sediment. Well yields in the upper Metolius River area outwash deposits generally range from 20 to $30 \mathrm{gal} / \mathrm{min}$, to approximately $200 \mathrm{gal} / \mathrm{min}$.

Reworked glaciofluvial material provides a locally important aquifer for domestic users in the vicinity of Tumalo (fig. 20). The source of that water is probably canal leakage. The unit thickness is generally less than $50 \mathrm{ft}$; however, thicknesses up to $200 \mathrm{ft}$ occur locally. Well yields from the reworked glacial deposits are generally less than $30 \mathrm{gal} / \mathrm{min}$.

A few wells have been drilled into glacial till (Qg on plate 1), which occurs exclusively within the Cascade Range. Those wells mostly serve youth camps and campgrounds. The deposits consist primarily of poorly sorted silt, sand, and gravel. The fine-grained matrix material and poor sorting of the deposits result in modest well yields of less than $30 \mathrm{gal} / \mathrm{min}$.

Quaternary sediment near the eastern and southern boundaries of the study area also serves as an important water-bearing unit. Robinson and Price (1963) described the Quaternary deposits near Prineville as fluviolacustrine sediment locally overlain by alluvium along the Crooked River and Ochoco Creek. The greatest total thickness of the fluviolacustrine unit is in excess of $250 \mathrm{ft}$ (fig. 20). The fluviolacustrine sediment sequence consists of an upper unit (up to $100 \mathrm{ft}$ thick) consisting primarily of sand with some gravel that overlies a fine-grained (mostly silt and fine sand) section (approximately $150 \mathrm{ft}$ thick) that in turn overlies a thin (approximately $10 \mathrm{ft}$ thick), relatively coarse sand and gravel unit. Remarkably, that general sequence is consistent over several square miles. The lower sand and gravel unit is artesian and is capable of yields of a few hundred gallons per minute. Wells producing from the upper coarse-grained unit have yields in the range of 10 to $20 \mathrm{gal} / \mathrm{min}$ and are mostly suitable for domestic supplies.
Robinson and Price (1963) documented higher head in the lower aquifer than in the shallow waterbearing unit. That phenomenon persists, at least locally, as exhibited in a pair of adjacent wells identified in this study. Well 14S/15E-15DAC1 (well no. 37, plate 1), completed to $206 \mathrm{ft}$, has a hydraulic head that is about $35 \mathrm{ft}$ higher than that in the adjacent shallow well 14S/15E-15DAC2 (well no. 38, plate 1) completed to $50 \mathrm{ft}$.

Recent alluvium (Qal) occurs adjacent to the Crooked River and Ochoco Creek. The alluvium may be as thick as 100 to $200 \mathrm{ft}$ in the vicinity of Prineville. However, it is nearly impossible to distinguish the younger sediment from the older Quaternary sedimentary strata using only well-log information.

The Millican valley is a small, east-trending subbasin containing a sequence of presumably Quaternary sediment (fig. 20). The subbasin is underlain by over $445 \mathrm{ft}$ of sand, clay, and gravel, and, near the basin margin, interbedded lava flows. The unit appears to be saturated only near the bottom of the sedimentary sequence, and wells typically yield less than $20 \mathrm{gal} / \mathrm{min}$.

The largest occurrence of Quaternary sediment within the upper Deschutes study area is in the vicinity of La Pine (fig. 20). This sedimentary sequence commonly consists of a lower unit of mostly silt and fine sand as much as several hundred feet thick, and an upper unit of silt, sand, and gravel as thick as $100 \mathrm{ft}$. Well drillers sometimes describe organic (woody) material within the lower unit. The organic component has been identified as a potential source of methane and hydrogen sulfide gases observed in some wells within the La Pine subbasin (Stephen R. Hinkle, U.S. Geological Survey, oral commun., 1999). A gamma $\log$ for well 23S/09E-36BBC (well no. 119, plate 1) located in the southernmost part of the La Pine subbasin (fig. 22A) reveals an irregular and mostly low gamma signature for the lower, fine-grained unit. The few spikes that occur on the log probably represent thin fallout tephra layers interbedded within the sedimentary sequence.

Few wells develop water from the lower, predominately fine-grained sedimentary unit. Low to moderate yields ( 5 to $30 \mathrm{gal} / \mathrm{min}$ ) are derived from interbedded sand and gravel layers in the unit, and moderate (50 to $90 \mathrm{gal} / \mathrm{min}$ ) yields come from interbedded lava flows and cinders. 
Most wells in the La Pine subbasin are constructed in the upper sediment unit. The upper unit differs from the lower unit in that the sediment is slightly coarser grained and lacks the organic debris characteristic of the lower unit. The uppermost part of the upper sediment also commonly contains pumice, probably reworked Mount Mazama tephra, although some may be derived from Newberry Volcano. A gamma log for a well (21S/11E-18CDA3) (well no. 114, plate 1) in the northeastern part of the La Pine subbasin (fig. 22B) clearly shows a thin layer at a depth

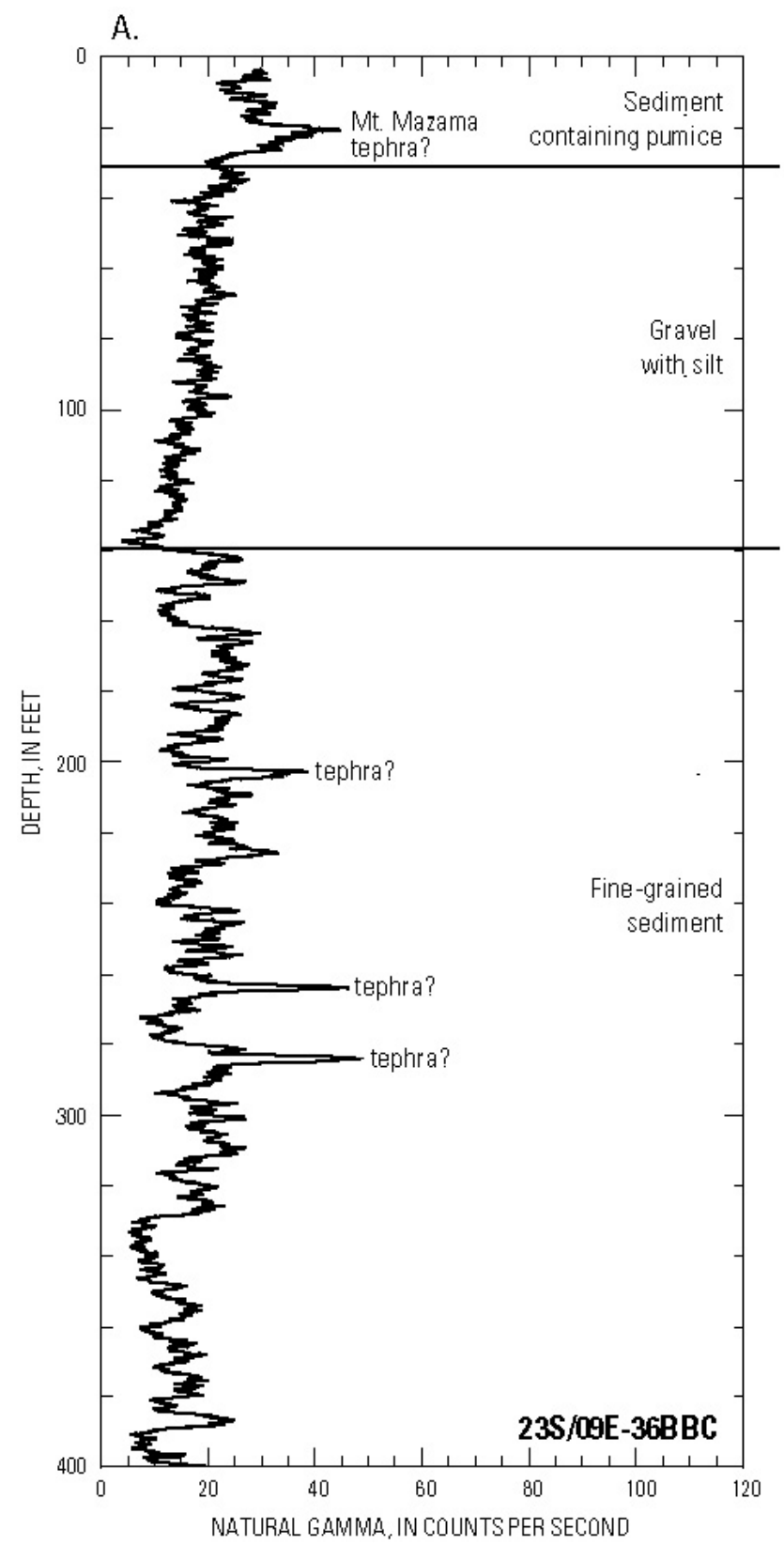

of about $10 \mathrm{ft}$ with a high gamma count characteristic of silicic tephra.

Well yields in the upper sedimentary unit are typically 10 to $30 \mathrm{gal} / \mathrm{min}$. The upper alluvium generally becomes coarser grained, however, in the northern part of the La Pine subbasin, resulting in well yields as great as $300 \mathrm{gal} / \mathrm{min}$. An estimate of hydraulic conductivity of the upper sedimentary unit is $88 \mathrm{ft} / \mathrm{d}$ based on aquifer tests conducted near La Pine (Century West Engineering Corporation, 1982). The tested wells produced from fine to coarse-grained sand with occasional fine gravel.

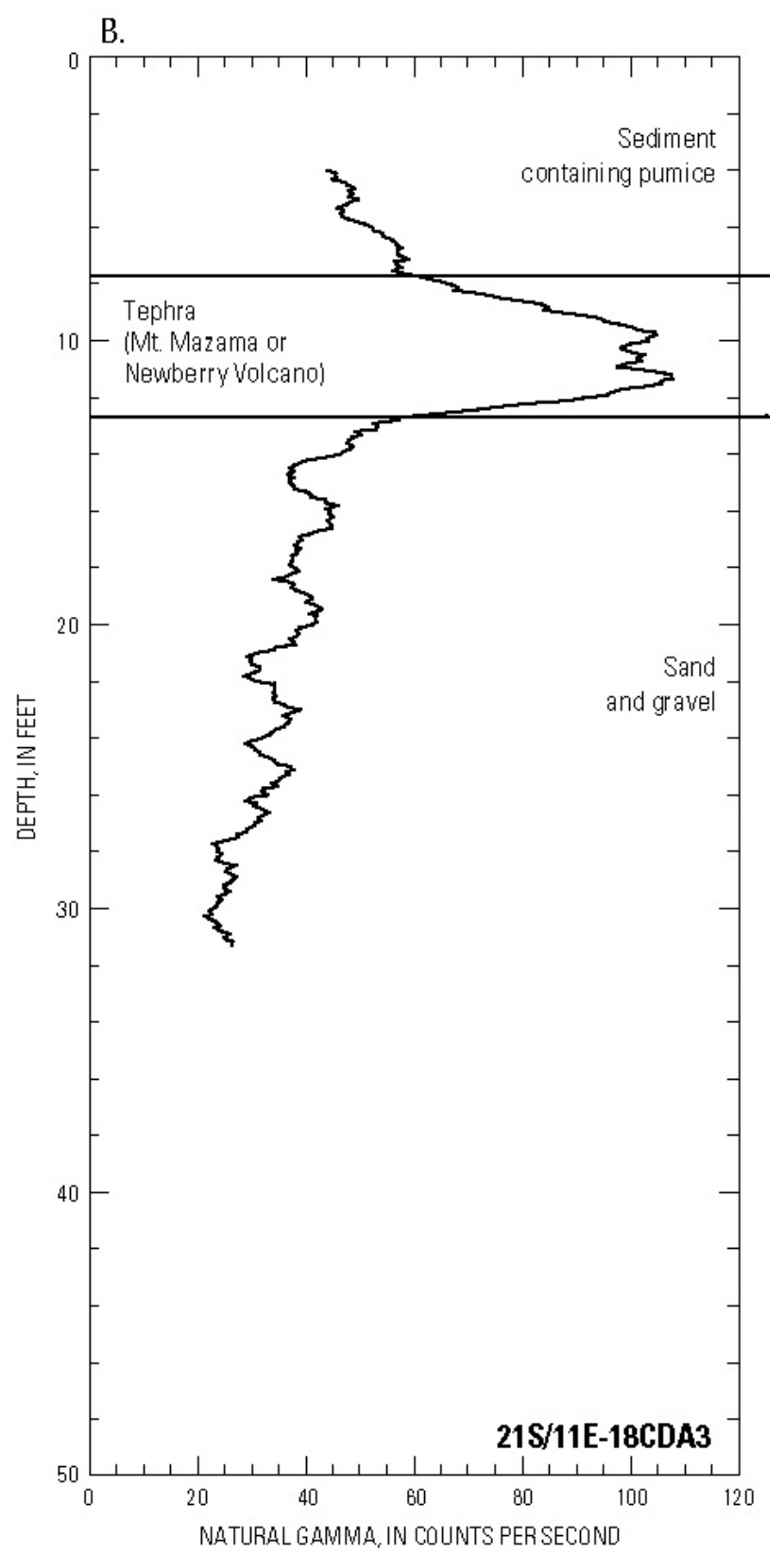

Figure 22. Natural gamma logs for wells penetrating Quaternary basin-fill deposits in the La Pine subbasin. A. Well in southernmost part of La Pine subbasin (23S/09E-36BBC, well no. 119, plate 1); B. Well in northern part of La Pine subbasin (21S/11E-18CDA3, well no. 114, plate 1). 


\section{HYDROGEOLOGIC UNITS}

For purposes of hydrologic analysis, geologic units are commonly combined or subdivided into groupings or subsurface regions, referred to as hydrogeologic units, characterized by distinct hydrogeologic properties. Hydrogeologic units are typically defined as having hydrologic or geologic properties that are distinct from surrounding units. Hydrogeologic units may consist of a single geologic unit, groups of geologic units, or discrete zones within a single geologic unit. All three cases occur in the upper Deschutes Basin.

Delineating hydrogeologic units in the upper Deschutes Basin is complicated by geologic heterogeneity and the lack of subsurface information over large areas. The large range of hydrologic conditions within single geologic units is difficult to specify in many instances.

Seven hydrogeologic units have been delineated in the upper Deschutes Basin (fig. 23): (1) Quaternary sediment, (2) permeable deposits of the Cascade Range and Newberry Volcano, (3) proximal lava flows of the Deschutes Formation, (4) the arc-adjacent alluvial-plain facies of the Deschutes Formation, (5) the ancestral Deschutes River-channel facies of the Deschutes Formation, (6) the inactive-margin facies of the Deschutes Formation, and (7) pre-Deschutes Formation rocks and hydrothermally altered rocks at depth beneath the Cascade Range and Newberry Volcano. The characteristics of the units are based on surficial geology and knowledge of subsurface conditions derived from well data. Boundaries between some of the hydrogeologic units are approximate because geologic contacts are gradational and subsurface data are sparse. Boundaries of other units are more precise because the geologic contacts are sharp and well defined by surface exposures. The hydrogeologic units are discussed in the following paragraphs of this section.

Coarse-grained surficial Quaternary sedimentary deposits constitute one hydrogeologic unit. This unit includes alluvial- and glacial-outwash deposits. These materials are generally permeable and productive where saturated. The fine-grained basin-filling deposits with low permeability that occur at depth in the La Pine graben are not considered part of this unit. Well yields are typically 10 to $300 \mathrm{gal} / \mathrm{min}$.

Permeable volcanic deposits of the Cascade Range and Newberry Volcano constitute another hydrogeologic unit. This unit consists primarily of permeable lava flows and minor pyroclastic and volcaniclastic interbeds, and comprises a large part of the upper Deschutes Basin. Hydrothermally altered rocks with low permeability that occur at depth beneath the Cascade Range and Newberry Volcano are not considered part of this unit. There may be areas within this unit that are hydrologically distinct, but no data were available on which to further subdivide this unit.

The Deschutes Formation can be divided into four hydrogeologic units. These units generally correspond to the depositional facies of the Deschutes Formation defined by Smith (1986b) and previously discussed.

- The most voluminous and areally extensive unit consists largely of proximal lava flows. It also includes undifferentiated volcanic deposits, primarily lava flows, not generally mapped as Deschutes Formation but of a similar age. This unit is generally permeable and locally is highly permeable. Many large-capacity public-supply wells in the Bend area produce from this unit. Well yields range up to $2,000 \mathrm{gal} / \mathrm{min}$.

- The second hydrogeologic unit in the Deschutes Formation corresponds to the arc-adjacent alluvial-plain facies. This unit consists of sediment interbedded with lava flows and ashflow tuff. This unit is more geologically heterogeneous than surrounding units, and hydrologic conditions are variable as a result. The unit is generally permeable and locally highly permeable. Many large-capacity irrigation wells in the Lower Bridge area produce from this unit. Well yields range up to $4,000 \mathrm{gal} / \mathrm{min}$.

- The third hydrogeologic unit within the Deschutes Formation corresponds to the ancestral Deschutes River channel facies. This unit consists predominately of coarse sand and gravel, distal parts of ash-flow tuffs, and intracanyon lava flows. It is generally highly permeable due to the large proportion of coarse-grained material and fractured basalt flows; for example, the very productive Pelton and Opal Springs basalt members. Many large-capacity wells in the Redmond area produce from both sediment and lava flows of this unit. Well yields range up to $2,300 \mathrm{gal} / \mathrm{min}$ near Redmond and up to 5,000 gal/min at Opal Springs. 


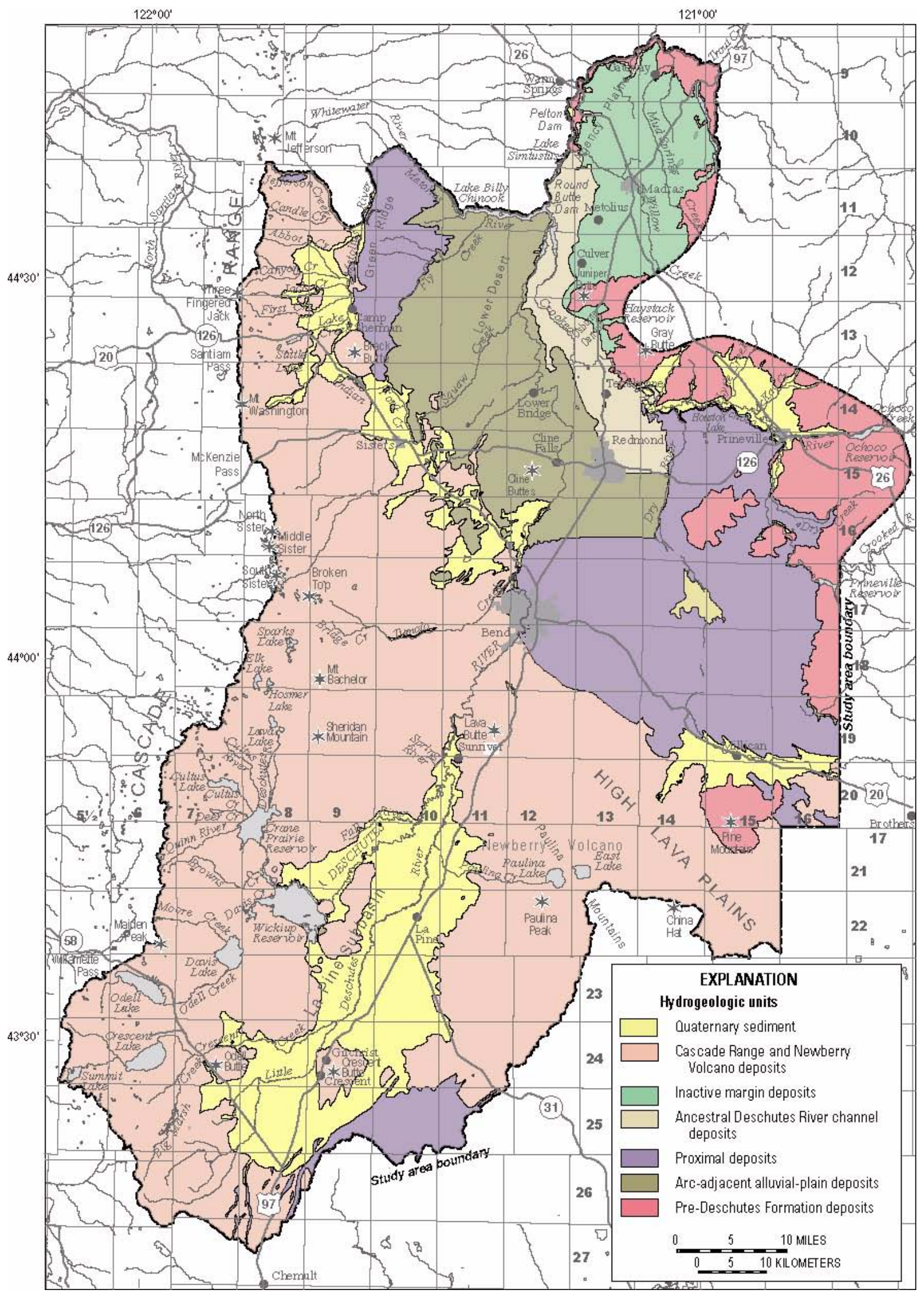

Figure 23. Hydrogeologic units in the upper Deschutes Basin, Oregon. 
- The fourth hydrogeologic unit within the Deschutes Formation corresponds to the inactivemargin facies. This unit consists of relatively finegrained clastic and pyroclastic material, much of which was derived from the John Day Formation. Because this unit consists largely of fine-grained, poorly sorted material, it has low permeability. Well yields from this unit are lower than yields from other units, ranging from 30 to $300 \mathrm{gal} / \mathrm{min}$.

The lowest hydrogeologic unit recognized in the upper Deschutes Basin consists of pre-Deschutes Formation rocks including the Prineville Basalt, John Day Formation, and hydrothermally altered rock with extremely low permeability at depth beneath the Cascade Range and Newberry Volcano. The Prineville Basalt has moderate to low permeability (with hydraulic conductivity ranging from 1 to $50 \mathrm{ft} / \mathrm{d}$ ), and the John Day Formation has extremely low permeability (with hydraulic conductivity ranging from 0.01 to $0.1 \mathrm{ft} / \mathrm{d}$ ). The John Day Formation and hydrothermally altered rocks are generally considered the boundary to regional ground-water flow system in the upper Deschutes Basin.

\section{SUMMARY}

The stratigraphic and structural framework of the upper Deschutes Basin influences many aspects of recharge, flow, and discharge of the ground-water flow system. Episodic volcanic activity in the region over the past several million years has resulted in a variety of volcanic, volcaniclastic, and volcanically derived sedimentary deposits. The volcanic activity and subsequent deposition and alteration of some deposits have produced strata that range widely in permeability.

Alteration and weathering of the John Day Formation rocks has resulted in very low permeability. The low-permeability rocks of the John Day Formation form a boundary to regional ground-water flow.

The Deschutes Formation is the most extensively used water-bearing unit in the study area. Depositional environments for the formation that were described by Smith (1986b) have proven to be useful hydrogeologic subdivisions. The ancestral Deschutes River deposits and some units within the arc-adjacent alluvial-plain region are among the highest yielding units within the Deschutes Formation. Yields of up to a few thousand gallons per minute have been observed from wells constructed into some of the ancestral Deschutes River deposits and parts of the arc-adjacent alluvial-plain region. Opal Springs basalt, Pelton basalt, and the rhyodacite dome complex near Steelhead Falls are particularly productive units and provide tens to hundreds of cubic feet per second of ground-water discharge to the Deschutes and Crooked Rivers, upstream of Round Butte Dam.

Ground-water recharge in the upper Deschutes Basin occurs chiefly in deposits of the Cascade Range and Newberry Volcano. The youthful and fractured character of the Cascade Range volcanic deposits facilitates rapid infiltration of precipitation and snowmelt in the Cascade Mountains. Additional recharge from canal leakage occurs along sections of unlined canals constructed near Bend into lava flows from Newberry Volcano.

Although surficial rocks and deposits of the Cascade Range and Newberry Volcano are very permeable in the upper Deschutes Basin, hydrothermal alteration and secondary mineralization have drastically reduced the permeability of the material at depth in those regions. The depth at which permeability is reduced is variable, ranging from 300 to $1,500 \mathrm{ft}$. Most ground-water flow in the Cascade Range and Newberry Volcano occurs above those depths.

Several small water-bearing regions occur throughout the study area within Quaternary sedimentary deposits. Glaciofluvial, fluvial, and fluviolacustrine deposits provide important water sources to communities such as Sisters, Prineville, La Pine, and Camp Sherman.

Tectonic structural features influence groundwater flow within the upper Deschutes Basin primarily by their influence on deposition. Several small depositional centers have formed along the base of fault-line scarps within the study area, resulting in accumulations of sand and gravel; Quaternary deposits in the vicinity of Camp Sherman and Sisters are good examples. The La Pine subbasin occupies a complex graben structure where up to $1,000 \mathrm{ft}$ of sediment was subsequently deposited by streams.

The variety of volcanic and tectonic processes within the upper Deschutes Basin has resulted in a highly heterogeneous accumulation of deposits.

Few distinct, well-defined hydrogeologic units have resulted from the geologic evolution of the area. However, knowledge about the general character of the deposits, their depositional environments, and areal distribution has allowed us to define the general distribution and range of hydraulic properties for the units. That distribution leads to a delineation of seven hydrogeologic zones within the upper Deschutes Basin. 


\section{REFERENCES CITED}

Allen, J.E., 1966, The Cascade Range volcano-tectonic depression of Oregon, in Transactions of the Lunar Geological Field Conference, Bend, Oregon, August 1965: Oregon Department of Mineral Industries, p. 21-23.

Baldwin, E.M., 1981, Geology of Oregon (3d ed.): Dubuque, Iowa, Kendall/Hunt Publishing Company, $170 \mathrm{p}$.

Bechtel Corporation, 1958, Round Butte Project Geologic Report: San Francisco, California, prepared for Portland General Electric Company, 34 p.

Blackwell, D.D., and Priest, G.R., 1996, Comments on "Rates and patterns of groundwater flow in the Cascade Range volcanic arc and the effect on subsurface temperatures" by S.E. Ingebritsen, D.R. Sherrod, and R.H. Mariner: Journal of Geophysical Research, v. 101, no. B8, p. 17,561-17,568.

Blackwell, D.D., Steele, J.L., Frohme, M.K., Murphey, C.F., Priest, G.R., and Black, G.L., 1990, Heat flow in the Oregon Cascade Range and its correlation with regional gravity, Curie point depths, and geology: Journal of Geophysical Research, v. 95, no. B12, p. 19,47519,493.

Bolke, E.L., and Laenen, Antonius, 1989, Ground-water inflow to the Deschutes River near the Warm Springs Indian Reservation, August 1985: U.S. Geological Survey Water-Resources Investigations Report 88-4184, 18 p.

Caldwell, R.R., and Truini, Margot, 1997, Ground-water and water-chemistry data for the upper Deschutes Basin, Oregon: U.S. Geological Survey Open-File Report 97-197, 77 p.

Century West Engineering Corporation, 1982, La Pine aquifer management plan: Bend, Oregon, prepared for Deschutes County, Oregon, variously paginated, several appendixes.

Conrey, R.M., 1985, Volcanic stratigraphy of the Deschutes Formation-Green Ridge to Fly Creek-northcentral Oregon: Corvallis, Oregon State University, M.S. thesis, 349 p.

Couch, R., and Foote, R., 1985, The Shukash and La Pine basins: Pleistocene depressions in the Cascade Range of central Oregon [abs]: Eos (American Geophysical Union, Transactions), v. 66, no. 3, p. 24.

Ferns, M.L., Lite, K.E., Jr., and Clark, M.D., 1996a, Lithologic controls on groundwater discharge to the Deschutes River between Lower Bridge and Lake Billy Chinook, central Oregon [abs.]: Geological Society of America Abstracts with Programs, v. 28, no. 5, p. 65 .

Ferns, M.L., Stensland, D.E., and Smith, G.A., 1996b, Geologic map of the Steelhead Falls quadrangle,
Deschutes and Jefferson Counties, Oregon: Oregon Department of Geology and Mineral Industries, Geological Map Series GMS-101, scale 1:24,000.

Gannett, M.W., Lite, K.E.,Jr., Morgan, D.S., and Collins, C.A., 2001, Ground-water hydrology of the upper Deschutes basin, Oregon: U.S. Geological Survey Water-Resources Investigations Report 00-4162, 77 p.

Hill, B.E., 1992, Stratigraphy and petrology of the Santiam Pass 77-24 drill core, Cascade Range, Oregon, in Hill, B.E., ed., Geology and geothermal resources of the Santiam Pass area of the Oregon Cascade Range, Deschutes, Jefferson, and Linn Counties, Oregon: Oregon Department of Geology and Mineral Industries Open-File Report O-92-3, p. 19-35.

Hooper, P.R., Steele, W.K., Conrey, R.M., Smith, G.A., Anderson, J.L., Bailey, D.G., Beeson, M.H., Tolan, T.L., and Urbanczyk, K.M., 1993, The Prineville Basalt, north-central Oregon: Oregon Geology, v. 55, no. 1, p. 3-12.

Ingebritsen, S.E., Sherrod, D.R., and Mariner, R.H., 1992, Rates and patterns of groundwater flow in the Cascade Range volcanic arc, and the effect on subsurface temperatures: Journal of Geophysical Research, v. 97, no. B4, p. 4,599-4,627.

Jay, J.B., 1982, The geology and stratigraphy of the tertiary volcanic and volcaniclastic rocks, with special emphasis on the Deschutes Formation, from Lake Simtustus to Madras in central Oregon: M.S. thesis, Oregon State University, Corvallis, Oregon, 119 p.

Johnson, D.M., Hooper, P.R., and Conrey, R.M., 1999, $\mathrm{XRF}$ analysis of rocks and minerals for major and trace elements on a single low dilution Li-tetraborate fused bead: Advances in X-ray Analysis, v. 41, p. 843-867.

Keith, T.E.C., Gannett, M.W., Eichelberger, J.C., and Waibel, A.F., 1986, Lithology and hydrothermal alteration of drill hole RDO-1, Newberry caldera, Oregon: Oregon Geology, v. 48, no. 9, p.103-107, 110.

Lawrence, R.D., 1976, Strike-slip faulting terminates the Basin and Range Province in Oregon: Geological Society of America Bulletin, v. 87, no. 6, p. 846-850.

MacLeod, N.S., and Sherrod, D.R., 1992, Reconnaissance geologic map of the west half of the Crescent $1^{\circ}$ by $2^{\circ}$ quadrangle, central Oregon: U.S. Geological Survey Miscellaneous Investigations Map I-2215, scale 1:250,000.

MacLeod, N.S., Sherrod, D.R., Chitwood, L.A., and Jenson, R.A., 1995, Geologic map of Newberry Volcano, Deschutes, Klamath, and Lake Counties, Oregon: U.S. Geological Survey Miscellaneous Investigations Map I-2455, scales 1:62,5000 and 1:24,000.

Manga, Michael, 1996, Hydrology of spring-dominated streams in the Oregon Cascades: Water Resources Research, v. 32, no. 8, p. 2,435-2,439. 
Manga, Michael, 1997, A model for discharge in springdominated streams and implications for transmissivity and recharge of Quaternary volcanics in the Oregon Cascades: Water Resources Research, v. 33, no. 8, p. $1,813-1,822$.

Norton, M.A., 1988, A hydrologic investigation of the Hay Creek area near Madras, Oregon: Oregon Water Resources Department, Ground Water OpenFile Report 88-02, 24 p. plus appendixes.

Oregon Climate Service, 1999, Climate data http://www.ocs.orst.edu/ocs_data.html, accessed July 14, 1999.

Peck, D.L., 1964, Geologic reconnaissance of the AntelopeAshwood area, north-central Oregon: U.S. Geological Survey Bulletin 1161-D, p. D1-D26.

Pitts, S., and Couch, R., 1978, Complete Bouguer gravity anomaly map of the Cascade Mountain Range, central Oregon: Oregon Department of Geology and Mineral Industries Geologic Map Series GMS-8, 1 sheet.

Robinson, J.W., and Price, D., 1963, Ground Water in the Prineville area, Crook County, Oregon: U.S. Geological Survey Water-Supply Paper 1619-P, 49 p.

Robinson, P.T., Brem, G.F., and McKee, E.H., 1984, John Day Formation of Oregon-A distal record of early Cascade volcanism: Geology, v. 12, no. 4, p. 229-232.

Sceva, J.E., 1968, Liquid waste disposal in lava terrane of central Oregon: U.S. Department of the Interior, Federal Water Pollution Control Administration, Technical Projects Branch Report No. FR-4, 66 p., plus a 96-page appendix.

Scott, W.E., 1977, Quaternary glaciation and volcanism, Metolius River area, Oregon: Geological Society of America Bulletin, v. 88, no. 1, p. 113-124.

Scott, W.E., and Gardner, C.A., 1992, Geologic map of the Mt. Bachelor volcanic chain and surrounding area, Cascade Range, Oregon: U.S. Geological Survey Miscellaneous Investigations Map I-1967, scale 1:50,000

Sherrod, D.R., 1991, Geologic map of a part of the Cascade Range between latitudes $43^{\circ}$ and $44^{\circ}$, central Oregon: U.S. Geological Survey Geologic Investigations Map I-1891, scale 1:125,000.

Sherrod, D.R., and Smith, J.G., 2000, Geologic map of upper Eocene to Holocene volcanic and related rocks of the Cascade Range, Oregon: U.S. Geological Survey Geologic Investigations Map I-2569, scale 1:500,000.

Sherrod, D.R., Taylor, E.M., Ferns, M.L., Scott, W.E., Conrey, R.M., and Smith, G.A., in press, Geologic map of the Bend 30- by 60-minute quadrangle, central Oregon: U.S. Geological Survey Geologic Investigations Map I-2683, scale 1:100,000.
Smith, G.A., 1986a, Simtustus Formation-Paleogeographic and stratigraphic significance of a newly defined Miocene unit in the Deschutes basin, central Oregon: Oregon Geology, v. 48, no. 6, p. 63-72.

Smith, G.A., 1986b, Stratigraphy, sedimentology, and petrology of Neogene rocks in the Deschutes basin, central Oregon - A record of continental-margin volcanism and its influence on fluvial sedimentation in an arc-adjacent basin: Corvallis, Oregon State University, Ph.D. dissertation, 467 p.

Smith, G.A., 1987, Geologic map of the Madras West and Madras East quadrangles, Jefferson County, Oregon: Oregon Department of Geology and Mineral Industries, Geological Map Series GMS-45, scale $1: 24,000$.

Smith, G.A., 1991, A field guide to depositional processes and facies geometry of Neogene continental volcaniclastic rocks, Deschutes basin, central Oregon: Oregon Geology, v. 53, no. 1, p. 3-20.

Smith, G.A., and Hayman, G.A., 1987, Geologic map of the Eagle Butte and Gateway quadrangles, Jefferson and Wasco Counties, Oregon: Oregon Department of Geology and Mineral Industries, Geological Map Series GMS-43, scale 1:24,000

Smith, G.A., Manchester, S.R., Ashwill, M., McIntosh, W.C., and Conrey, R.M., 1998, Late Eocene-early Oligocene tectonism, volcanism, and floristic change near Gray Butte, central Oregon: Geological Society of America Bulletin, v. 110, no. 6, p. 759-778.

Smith, G.A., Snee, L.W., and Taylor, E.M., 1987, Stratigraphic, sedimentologic, and petrologic record of late Miocene subsidence of the central Oregon High Cascades: Geology, v. 15, no. 5, p. 389-392.

State of Oregon, 2001, Oregon Blue Book 2001-2002: Salem, Oregon, $465 \mathrm{p}$.

Stearns, H.T., 1931, Geology and water resources of the middle Deschutes River basin, Oregon: U.S. Geological Survey Water-Supply Paper 637-D, p. 125-212.

Swanberg, C.A., Walkey, W.C., and Combs, Jim, 1988, Core hole drilling and the "rain curtain" phenomenon at Newberry Volcano, Oregon: Journal of Geophysical Research, v. 93, no. B9, p. 10,163-10,173.

Swanson, D.A., 1969, Reconnaissance geologic map of the east half of the Bend quadrangle, Crook, Wheeler, Jefferson, Wasco, and Deschutes Counties, Oregon: U.S. Geological Survey Miscellaneous Investigations Map I-568, scale 1:250,000.

Taylor, E.M., 1981, Central High Cascade roadside geology -Bend, Sisters, McKenzie Pass, and Santiam Pass, Oregon, in Johnston, D.A., and Donnelly-Nolan, J.M., eds., Guides to some volcanic terranes in Washington, 
Idaho, Oregon, and northern California: U.S. Geological Survey Circular 838, p. 55-83.

Taylor, G.H., 1993, Normal annual precipitation, State of Oregon: Corvallis, Oregon State University, Oregon Climate Service, map.

Uppuluri, V.R., 1974, Prineville chemical type: A new basalt type in the Columbia River Group: Geological Society of America Bulletin, v. 85, no. 8, p. 1,315-1,318.

Walker, G.W., 1969, Geology of the High Lava Plains Province, in Weissenborn, A.E., ed., Mineral and Water Resources of Oregon: Oregon Department of Geology and Mineral Industries Bulletin 64, p. 77-79.
Walker, G.W., Peterson, N.V., and Greene, R.C., 1967, Reconnaissance geologic map of the east half of the Crescent quadrangle, Lake, Deschutes, and Crook Counties, Oregon: U.S. Geological Survey

Miscellaneous Geological Investigations Map I-493, scale 1:250,000.

Walker, G.W., and Nolf, Bruce, 1981, High Lava Plains, Brothers to Harney basin, Oregon, in Johnston, D.A., and Donnelly-Nolan, J.M., eds., Guides to some volcanic terranes in Washington, Idaho, Oregon, and northern California: U.S. Geological Survey Circular 838, p. 105-111. 
